Strategic Environmental Research and Development Program ER-1459

\title{
Metal Ion Sensor with Catalytic DNA in a Nanofluidic Intelligent Processor
}

Donald M. Cropek, Tulika S. Dalavoy, Paul W. Bohn, Yi Lu,

December 2011 Jonathan V. Sweedler, and Mark A. Shannon

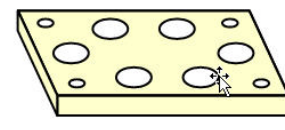

polycarbonate block

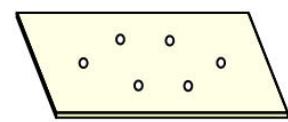

PMMA via reduction layer

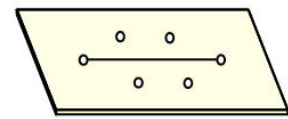

separation channel layer

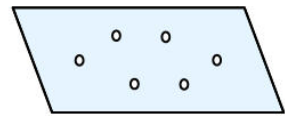

nanoporous polycarbonate

track-etched membrane

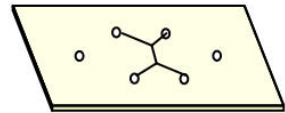

detection channel layer
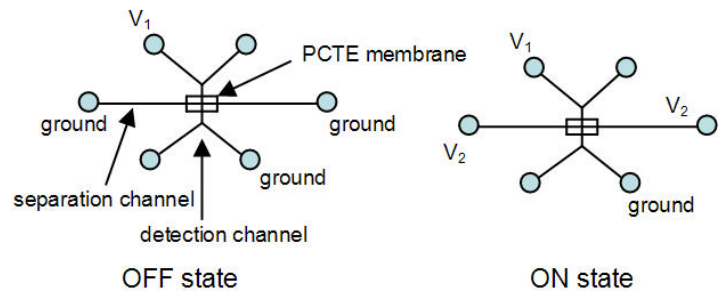

ON state 


\section{Metal Ion Sensor with Catalytic DNA in a Nanofluidic Intelligent Processor}

Donald M. Cropek

Construction Engineering Research Laboratory

U.S. Army Engineer Research and Development Center

2902 Newmark Drive

PO Box 9005

Champaign, IL 61826-9005

Tulika S. Dalavoy, Paul W. Bohn, Yi Lu, and Jonathan V. Sweedler

University of Illinois

Department of Chemistry

600 S. Mathews Avenue

Urbana IL 61801

Mark A. Shannon

University of Illinois

Department of Chemical and Biomolecular Engineering

105 S. Mathews Avenue

Urbana IL 61801

Final report

Approved for public release; distribution is unlimited.

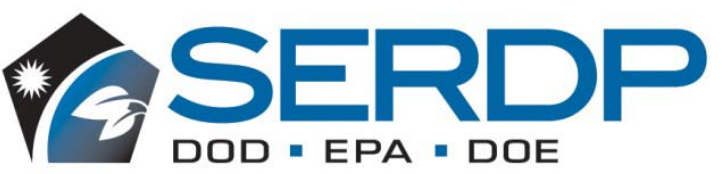

Prepared for Strategic Environmental Research and Development Program 901 N Stuart Street, Suite 303

Arlington, Virginia 22203

Under ER-1459 


\section{Abstract}

Due to the numerous toxicological effects of lead, its presence in the environment needs to be effectively monitored. Incorporating a biosensing element within a microfluidic platform enables rapid and reliable determinations of lead at trace levels. This work produced a microchip-based lead sensor that employs a lead-specific DNAzyme (also called catalytic DNA or deoxyribozyme) as a recognition element that cleaves its complementary substrate DNA strand only in the presence of cationic lead $\left(\mathrm{Pb}^{+}\right)$. Fluorescent tags on the DNAzyme translate the cleavage events to measurable, optical signals proportional to $\mathrm{Pb2}+$ concentration. The DNAzyme responds sensitively and selectively to $\mathrm{Pb}^{+}$, and immobilizing the DNAzyme in the sensor permits both sensor regeneration and localization of the detection zone. The immobilized DNAzyme retains its $\mathrm{Pb} 2+$ detection activity in the microfluidic device and can be regenerated and reused. The particular DNAzyme shows no response to other common metal cations and the presence of these contaminants does not interfere with the lead-induced fluorescence signal. Attempts were made to incorporate a second DNAzyme with selectivity for uranium in the same microfluidic chip sensor, to demonstrate multiplexing capabilities for multiple metal analytes in a single injection. Crosstalk between the lead DNAzyme and the uranium DNAzyme severely limited the utility of this multi-analytic chip.

DISCLAIMER: The contents of this report are not to be used for advertising, publication, or promotional purposes. Citation of trade names does not constitute an official endorsement or approval of the use of such commercial products. All product names and trademarks cited are the property of their respective owners. The findings of this report are not to be construed as an official Department of the Army position unless so designated by other authorized documents. 


\section{Table of Contents}
Abstract
ii

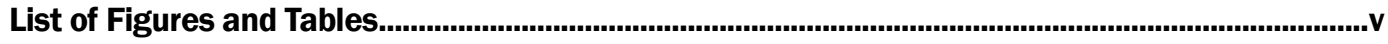

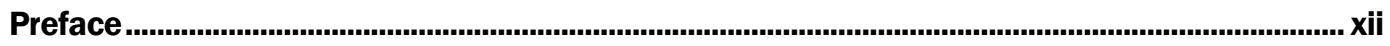

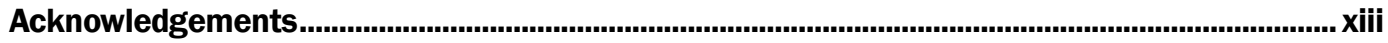
Unit Conversion Factors ...........................................................................................................xiv

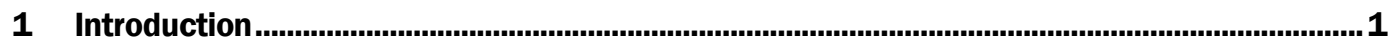

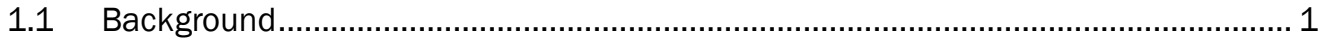

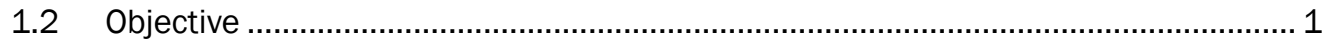

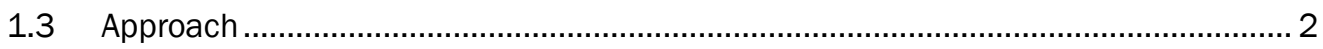

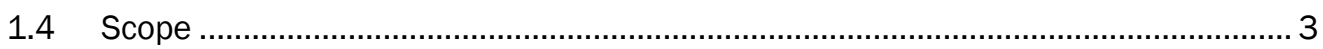
1.5 Mode of Technology Transfer .................................................................... 3

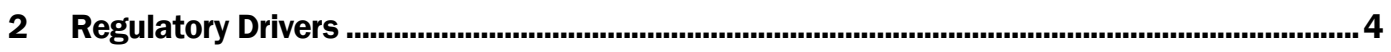

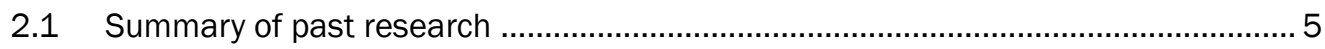

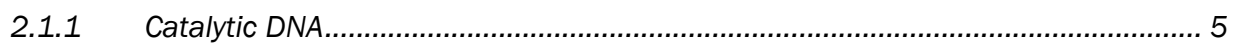

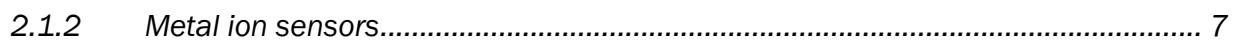

2.1.3 Microfabricated analysis systems................................................................... 8

2.1.4 Fluidic control with molecular gates ................................................................... 8

2.1.5 Miniaturized microfluidic lead sensor................................................................ 9

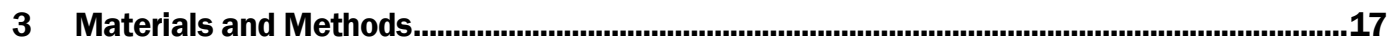

3.1 General chemicals and materials................................................................... 17

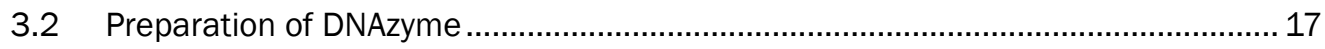

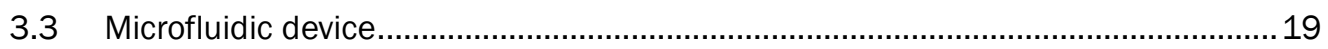

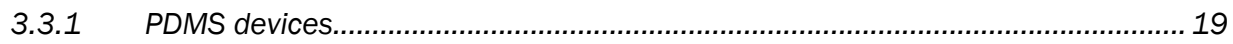

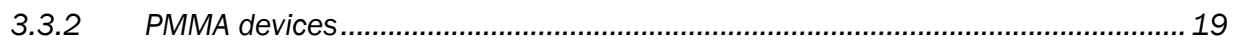

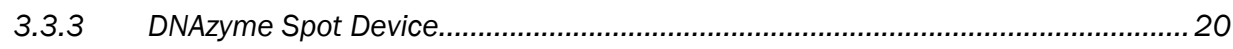

3.4 DNAzyme immobilization ............................................................................. 21

3.5 Hybridization and detection of lead.................................................................... 22

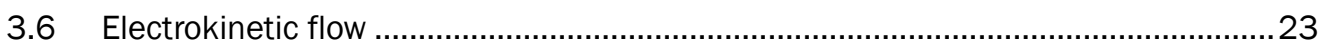

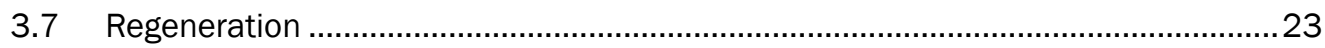

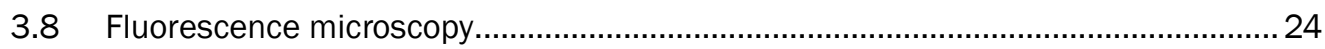

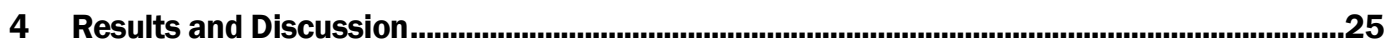

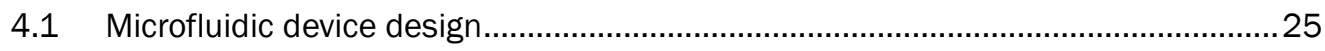

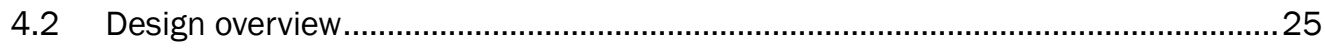

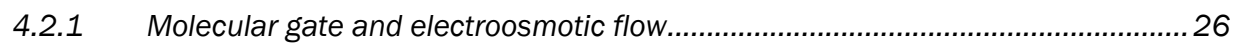

4.2.2 Design problems ............................................................................................ 29

4.2.3 Increased device failure rate: cause and effect............................................... 39 
4.3 Separation and detection of amino acids ....................................................40

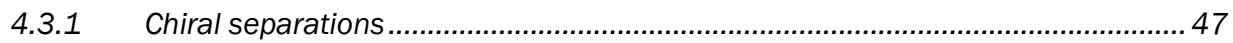

4.4 Microfluidic devices with Au-coated NCAMs ...................................................48

4.4.1 Reducing the effect of Au coating........................................................................ 52

4.4.2 Applications of NCAMs in affinity-based separations ........................................... 53

4.5 Immobilization of DNAzyme on solid supports ................................................64

4.5.1 Surface modification of PMMA using aminated DNA ...........................................68

4.5.2 Immobilization of DNAzyme on SuperAldehyde glass.......................................... 71

4.5.3 Immobilization of DNAzyme on superstreptavidin glass........................................ 75

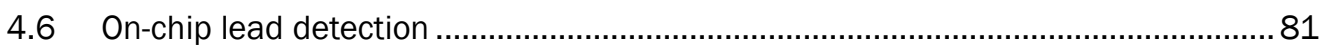

4.6.1 Fluorescence on a PMMA microfluidic device .................................................. 81

4.6.2 Glass microbeads for greater interaction ........................................................... 84

4.7 Electrochemical detection of DNAzyme activity...............................................90

4.8 Immobilization of DNAzyme on fluorous-coated silica in PDMS channel..................91

4.9 A new DNAzyme specific for uranium ....................................................................94

4.10 Multianalyte device: detection of lead and uranium ions in a

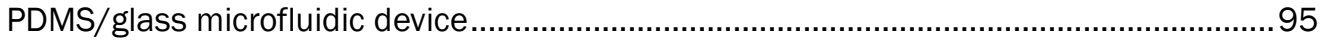

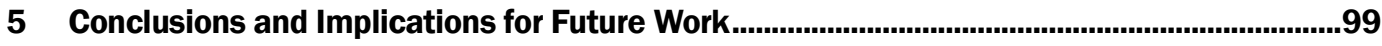

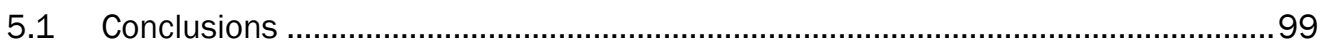

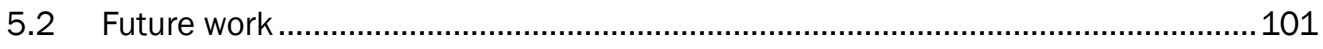

Literature Cited in Report ............................................................................................................. 104

Appendix B: List of Publications Produced During the Project ....................................................108

Appendix C: Other Supporting Material ..............................................................................111

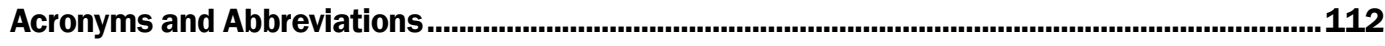

Report Documentation Page 


\section{List of Figures and Tables}

\section{Figures}

Figure 1. (A) Structure of the Pb-sensing catalytic DNA and (B) fluorosensing mechanism and results.

Figure 2. Structure of the microfluidic device for heavy metal detection. ........................................... 9

Figure 3. DNAzyme selectivity compared to common divalent cationic species.

Figure 4. Schematic of a three-dimensional NAl gateable microfluidic device. Both crossed-microfluidic channels are identical with dimensions of 50- $\mu \mathrm{m}$ width, $30-\mu \mathrm{m}$ depth, and 14-mm length.

Figure 5. Temporal sequence of fluorescence images at the intersection of the crossed microchannels. Source channel (horizontal) was filled with $1 \mu \mathrm{M} \mathrm{Pb}{ }^{2+}$ in BGE $(25 \mathrm{mM}$ lactic acid, $25 \mathrm{mM}$ HEPES, $50 \mathrm{mM} \mathrm{NaCl}$ ) and receiving channel (vertical) was filled with hybridized DNAzyme in BGE. Dashed lines indicate the position of the horizontal source channel. In the off-state (A), all reservoirs were floated while the on-state (B), causes injection of $\mathrm{Pb}^{2+}$ solution. Lead solution is transferred from the source channel, across the NAl toward grounded reservoir. The reaction with DNAzyme produced fluorescence from cleaved DNA in the receiving channel (C). During on state bias, the DNA cleavage reaction reached equilibrium and a constant fluorescent signal is maintained (captured image is $\sim 40 \mathrm{~s}$ after on-state bias is applied). $\mathrm{Pb}^{2+}$ plug continued to move to the ground electrode and cleaved DNA moved toward the positive bias. Image (D) is $\sim 1 \mathrm{~s}$ after off state bias is applied and (E) is $\sim 40 \mathrm{~s}$ after off state bias is applied. Cleaved DNA moved toward the positive bias and the receiving channel was flushed with bulk hybridized DNAzyme solution. Repetitive $\mathrm{Pb}^{2+}$ plug injection after switching back to the on-state bias is shown in $(\mathrm{F})$.

Figure 6. Plot of fluorescence enhancement as a function of lead ion concentration from $0.1,1,5,10,50,100$, and $200 \mu \mathrm{M}$. Error bars represent $\pm 1 \sigma(n=4)$.

Figure 7. Typical fluorescence signals during repetitive $\mathrm{Pb}^{2+}$ injection (100 nM in BGE). Detection was performed at the intersection of crossed channels. On state was maintained for $20 \mathrm{~s}$ and switched to off state for $10 \mathrm{~s}$, repetitively...

Figure 8. Plot of fluorescence enhancement as a function of lead ion concentration spiked in the electroplating sludge sample for standard addition calibration. Error bars represent $\pm 1 \sigma(n=4)$.

Figure 9. The sensor is composed of a dual-labeled cleavable substrate DNA whose 5'and 3 '-end is labeled with a fluorophore (FAM) and a quencher (Dabcyl), respectively, and an enzyme strand whose 3'-end is labeled with a Dabcyl. Initially, the fluorescence of FAM is quenched because of the close proximity of the Dabcyl. In the presence of $\mathrm{Pb}^{2+}$, the substrate DNA is cleaved, resulting in the release of fragments and a concomitant increase in fluorescence.

Figure 10. Schematic diagram showing the various layers in the PMMA microfluidicnanofluidic device and the potential program used to control fluid flow between the channels. (Top) Exploded schematic diagram showing the constituent layers individually. (Bottom) Diagram showing terminals and voltage programs used to realize the OFF and ON states. The central rectangle represents the NCAM (PCTE membrane) that controls fluidic communication between microfluidic layers.

Figure 11. Design for fabrication of microfluidic chip for lead sensing. 
Figure 12. Three molecular gate devices with 10, 50, and $220 \mathrm{~nm}$ diameter pore NCAMs (from left to right, respectively). The NCAM extends across the entire device and is positioned between the cross channels and over the separation channel.

Figure 13. Laser-induced fluorescence (LIF) detection of $1 \mu \mathrm{M}$ GFP in $10 \mathrm{mM}$ phosphate buffer injected for $2 \mathrm{~s}$ at $600 \mathrm{~V}$ from one microfluidic channel, across a NCAM, and into a second microfluidic channel containing the buffer solution only.

Figure 14. Double membrane samples with three microfluidic layers separated in the vertical direction by two NCAM layers. The device on the left has two $50 \mathrm{~nm}$ NCAMs and the device on the right has two $100 \mathrm{~nm}$ NCAMs. The separation channel is located between the NCAM layers with an injection and a collection channel at both ends.

Figure 15. Relief schematic of a device showing the routing scheme of the microfluidic channels relative to each other. On the right is a cross-section of the intersection region where nanofluidic interconnects are formed between the microfluidic layers.

Figure 16. Glass capillary electrophoresis data demonstrating that distinct separation between the lead and copper bands is possible.

Figure 17. Schematic of the new microfluidic device design showing the two parallel plate capacitors located in the middle of the separation channel. The cross-sectional view shows the intended vertical overlap of one of the capacitive detectors.

Figure 18. Left, electrical schematic used for detection, and on the right, its equivalent circuit, a low-pass filter. The modified Wheatstone bridge has the greatest $V_{\text {out }}$ sensitivity at the cutoff frequency of the equivalent circuit.

Figure 19. The four-step process to bond a second layer (already processed) to a device with a first layer. The second PMMA layer is affixed to a coverglass carrier for processing, and since the coverglass is much thicker than the PMMA ( $200 \mu \mathrm{m}$ to $20 \mu \mathrm{m}$ ), it expands and contracts at the same CTE as the coverglass, which leads to layer misalignment.

Figure 20. Modification of the SU8 formulation used to make individual microfluidic layers reduces both the number and size of the cracks emanating from the sides of the microfluidic channels by approximately a factor of 3 , and reduces the thickness variability of the layer by approximately a factor of 4

Figure 21. Choice of the temporary carrier substrate material has the tradeoff between forming a flat layer, although highly stressed on glass, or an unstressed layer but highly non-uniform on a polymer substrate. Because the polymer substrate has a lower rigidity than glass, it needed to be thicker, and since it was both thicker and had a lower thermal conductivity, it bowed during hotplate processing.

Figure 22: Current temporary substrate carrier construct consisting of a bottom rigid glass layer followed by a flexible stress accommodating PDMS layer and, on top, a rigid SU8 layer for CTE matching to SU8 device layers.

Figure 23. Current carrier substrate used to pattern a crack free SU8 layer where a release layer is coated first and cured, followed by the SU8 layer which is processed into a device layer.

Figure 24. Microscope images of (A) cracks forming when SU8 is processed directly onto glass and (B) a crack-free layer of SU8 when it is processed on our latest carrier substrate.

Figure 25. Example of a device made using SU8 and showing intact metal electrode layers.

Figure 26. Polymers with a higher level of cross-linking appeared to have fewer pinholes, or pinholes of shorter depth; greater thermal stress on lower cross-linked polymers also seems to increase pinhole depth and density. 
Figure 27. A schematic drawing of a PMMA multi-layer microfluidic device with associated thickness of each layer of the device.

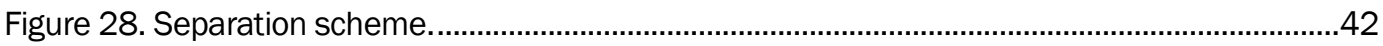

Figure 29. Conceptual sketch of the experimental setup. ……………………………………....42

Figure 30. Voltage configuration............................................................................................43

Figure 31. Collection of FITC-labeled aspartate for the third injection...............................................44

Figure 32. Collection of FITC-labeled serine for the third injection..................................................44

Figure 33. Demonstration of collected peak of aspartate. ..............................................................45

Figure 34. Separation of 5 amino acids in $20 \mathrm{mM}$ borate buffer $\mathrm{pH}$ 9.3. The electropherogram was from a series of multiple injections. The maximum peak intensity of the first peak was observed in $39 \mathrm{~s}$ after the second injection. The peak marked with the arrow on the electropherogram is an unknown impurity. The separation orders of the analyte peaks are assigned as shown in Table 4.

Figure 35. Electropherogram collected with the new test device. The electropherogram was from a series of multiple injections. The first peak showed maximum intensity after $25 \mathrm{~s}$.

Figure 36. Multi-dimensional separation on a PMMA hybrid microfluidic/nanofluidic device. LIF1 is the first stage separation data and LIFO is the second stage separation data.

Figure 37. Schematic diagram of potential distribution along a microfluidic channel in contact with a gold membrane. The illustration at the top of this figure represents one microfluidic channel and the Au-NCAM in cross-section. The electric field in the microfluidic channel, $\mathrm{dV} / \mathrm{dx}$, is smaller above the Au membrane due to the high conductance through the Au.

Figure 38. Fabrication details. A: electroless deposition; B: patterning of NCAM using $\mathrm{KI} / \mathrm{I}_{2}$ etchant; C: assembling PDMS device using rapid prototyping; D: brightfield micrograph of microfluidic device at intersection of microfluidic channels; the Au-coated region appears as a black region in the image. Width of channel and gold feature are 50 and $280 \mu \mathrm{m}$, respectively.

Figure 39. Current distribution on gold, $\Delta V \sim 3 V$. Cathodic current is represented as a positive current.

Figure 40. Au-coated NCAM architectures: (1) continuously coated; (2) coated on one side; (3) gold island film coated NCAMs. Mathematical signs indicate applied potential across the membrane, and arrows indicate the direction of electrokinetic transport.

Figure 41. Injection across gold coated 400 nm NCAMs...............................................................53

Figure 42. Immobilization of Fab'.

Figure 43. SEM micrograph of the inner pore surface of Au-IgG-Fab'-Au-NCAM and controls: (A) the surface of the pores with Au-lgG-Fab'-Au; (B) schematic representation of Au-lgG-Fab'-Au-NCAM, the antibody-based molecular recognition gate for (A); (C) cleaned Au-NCAM; and (D) control Au-NCAM.

Figure 44. MALDI-TOF MS spectra of standard solution of $5 \mu \mathrm{M}$ Aplysia acidic peptide (control antigen: AP) and human insulin (antigen: IN), 5 pmole each on the probe (A); spectrum of $500 \mathrm{nM}$ of AP and $500 \mathrm{nM}$ of IN solution prepared in release buffer without BSA blocker, 0.5 pmole each on the probe (B); on-probe release of captured peptide from Fab'-Au-NCAM (C); and on-probe release of captured peptide from control Au-NCAM (D). $\mathrm{m} / \mathrm{z}$ of peaks are (i) 2961.5, (ii) 5809.1, (iii) 2904.9, (iv) 3886.9, (v) 5809.6, (vi) 2912.3, (vii) 3886.1 and (viii) 5807.1. 
Figure 45. Multimodal concept for Au NCAM shown from top left, (a) and (b): microfluidic element in flow-past and flow-through configuration, respectively; (c) plasmonic chemical sensor.

Figure 46. Optical test bed for characterization of subwavelength transmission. This setup enables characterization of both spatial $(\theta, \Phi)$ and spectral $(\lambda)$ information by tuning the Ti:sapphire single band source.

Figure 47. Au NCAMs milled in a $200 \mathrm{~nm}$ thick Au film on glass. In all cases, the pores are $230 \mathrm{~nm}$ in diameter and are milled in a $25 \times 25$ square array. Periodicity $a_{0}$ (distance center-to-center) is varied as follows: (a) $a_{0}=570 \mathrm{~nm}$, (b) $a_{o}=670 \mathrm{~nm}$, and (c) $a_{0}=710$ $\mathrm{nm}$.

Figure 48. (a) Schematic diagram of the device (i) and the electric field extension across the NCA (ii); (b) images showing FL2- distribution during the floating injection and separation processes: (i) after floating injection from the sample (S/SW) microfluidic channel to the separation (B/BW) channel; and (ii) immediate application of separation potential (in the B/BW channel).

Figure 49. Concentration polarization and analyte band concentration demonstrated by using fluorescein ( $\left.\mathrm{FL}^{2}\right)$ in $10 \mathrm{mM}$ phosphate buffer, $\mathrm{pH}$ 8.0. (A) is a fluorescence microscope image where (a) is a microfluidic channel above the NCA and (b) a microfluidic channel below the NCA; (i) shows ion depletion, (ii) shows ion accumulation and (iii) shows the focused analyte band. (B) and (C) are LIF data with different detection points. For (B), LIFO is focused at (ii) and LIF1 is focused at (i). For (C), LIFO and LIF1 are both focused along channel (b) to detect band movement (iii).

Figure 50. Fluorescein dynamics in a source channel. Attempted injection across $400 \mathrm{~nm}$ polycarbonate membrane coated with continuous gold film. There is no appreciable injection of fluorescein from the source channel into the receiving channel.

Figure 51. Finite element simulation of the electrokinetic flow near an Au segment in a polymer microchannel. In all three plots, red arrows depict the velocity field (electroosmotic), while black contour lines are lines of constant electrical potential. In (a), the color surface plot depicts the electric potential; in (b), color depicts electric field strength. The peak field strength occurs just at the edges of the gold patch, where it reaches $160 \mathrm{~V} \mathrm{~cm}^{-1}$. (c) A magnified view of the left corner of the Au patch; the black arrow depicts the relative size of the electrophoretic velocity for fluorescein (electrophoretic mobility $=3.5 \mathrm{~cm}^{2} \mathrm{~V}^{-1} \mathrm{~s}^{-1}$ ) near the edge of the gold segment.

Figure 52. Injection across NCAM coated on one side. Each trace shows representative injections using intermediate (top) and small (bottom) bias.

Figure 53. Simulation of EOF pumping across a single nanopore. Receiving side of NCAM is coated with $\mathrm{Au}$. Color scale represents potential distribution within microchannel, and arrows represent flow field.

Figure 54. Fluorescence microscopy illustrating injection through an Au island-coated NCAM. Right: a single frame from an injection movie. The source channel is visible as the horizontal fluorescent area interrupted by a dark diagonal (Au-coated region). The receiving channel bias alternated between floating and $-30 \mathrm{~V}$ relative to the source channel to accomplish injection. Left: a sequence of injections was followed by correlating the fluorescence intensity (left, bottom) integrated over the region of interest (yellow square), with the applied potential pulse train (left, top).

Figure 55. Schematic of the ratiometric internal control method before and after $\mathrm{Pb}^{2+}$ reaction to monitor non-specific release of substrate from immobilized enzyme. Cleavable substrate is shown as purple oligos with green heads, uncleavable as black oligos with red heads, and enzyme as blue oligos. Backfilling of the mercaptohexanol (MCH) monolayer is represented by short red lines. 
Figure 56. NCAM pore diameter effect on immobilized DNAzyme activity. $200 \mathrm{~nm}$ pore diameter NCAM has increased surface area compared to $600 \mathrm{~nm}$ due to higher pore density but has less activity. The decrease in activity is attributed to decreased diffusion and less active DNAzyme complex because of pore constraints. Uncleavable Alexa546 intensity is shown in gray; cleavable fluorescein in green; and the ratio of Fl/Alexa in red. Error bars represent one standard deviation of three independent reactions.

Figure 57. Immobilization of DNA on PMMA.

Figure 58. Immobilization of amine functionalized DNA [Am-PMMA(12)-FI] on PMMA substrate by Soper's method, showing fluorescence images: (A) DNA spotted on chemically activated PMMA; (B) control 1, non-aminated DNA on chemically activated PMMA; (C) control 2, Am-PMMA(12)-Fl on non-activated, cleaned PMMA substrate; (D) hybridized DNA on PMMA; and (E) image on unhybridized DNA spot taken at the same time as image $\mathrm{D}$.

Figure 59. Microarray image of 50 spots of DNAzymes attached to the SuperAldehyde glass. Each row is a different DNA sequence immobilized as detailed below (from the top):

Figure 60. Immobilization of amine functionalized DNA [Am-PMMA(12)-FI] on SuperAldehyde glass: (A) $20 \mu \mathrm{M}$ DNA in 3X SSC; (B) $20 \mu \mathrm{M}$ DNA in 1X Microspotting Solution Plus; (C) $100 \mu \mathrm{M}$ DNA in 3X SSC; (D) $100 \mu \mathrm{M}$ DNA in 1X Microspotting Solution Plus; and (E) control-spot of non-aminated DNA.

Figure 61. Immobilization of fluorescein-tagged, biotin modified DNAzyme (Bio-17E-FI) on superstreptavidin glass (SSG) substrate: (A) DNAzyme on SSG; (B) control for non-specific binding using Am-PMMA(12)-Fl on SSG; (C) surface after hybridization; and (D) surface after reaction with $\mathrm{Pb}^{2+}$.

Figure 62. The effect of TCEP used for breaking disulfide linkages on immobilization of DNAzyme enzyme strand. TCEP has no effect on total DNAzyme immobilization as evidenced by the equal amounts of uncleavable standardization strand for all cases. Months-old TCEP does diminish the activity of the DNAzyme relative to fresh and TCEP absent samples but the effect on the oligo is not understood. The TCEP must directly alter the enzyme strand since numerous rinsing steps before $\mathrm{Pb}^{2+}$ reaction should completely remove TCEP from the NCAM DNAzyme surface. Error bars represent one standard deviation of three independent reactions.

Figure 63. Activity of DNAzyme on NCAM, in the absence and presence of a backfilling $\mathrm{MCH}$ monolayer. The $\mathrm{MCH}$ monolayer causes a $\sim 3.5$ fold increase in activity by creating more active DNAzyme complexes. Uncleavable Alexa 546 intensity is shown in gray, cleavable fluorescein in green, and the ratio of Fl/Alexa in red. Error bars represent one standard deviation of four independent reactions.

Figure 64. $\mathrm{NaCl}$ effect on DNAzyme activity at various concentrations in reaction solution. System is shown to be optimized at $50 \mathrm{mM} \mathrm{NaCl}$, with lower concentrations resulting in unstable complex and higher concentrations inhibiting cleaved fragment release. Uncleavable Alexa 546 intensity is shown in gray, cleavable fluorescein in green, and the ratio of $\mathrm{Fl} /$ Alexa in red. Error bars represent one standard deviation of three independent reactions.

Figure 65. Specificity of $\mathrm{Pb}^{2+}$ DNAzyme on NCAM versus other divalent metal ions: $\mathrm{Pb}^{2+}$ shows highest activity, however some specificity of the DNAzyme is lost compared to the native $17 \mathrm{E} / 17 \mathrm{~S}$ system; uncleavable Alexa546 intensity is shown in gray, activity ratio (FI/Alexa) in presence of $\mathrm{Pb}^{2+}$ in red and other divalent metal ions in blue. Error bars represent one standard deviation of three independent reactions.

Figure 66. The effect on DNAzyme activity of enzyme poly-T linker length between 5 -thiol and first complimentary base. While no dependence on linker length is observed for OT, 
5T, or 15T, a drastic decrease in activity is observed in the 10T system though the cause is undetermined. Uncleavable Alexa 546 intensity is shown in gray, cleavable fluorescein in green, and the ratio of Fl/Alexa in red. Error bars represent one standard deviation of four independent reactions.

Figure 67. Study of activity of immobilized DNAzyme in the detection channel of the PMMA microfluidic-nanofluidic device: (A) unmodified channel; (B) immobilized enzyme strand; (C) after hybridization with quencher-bearing substrate strand; (D) after injection of $10 \mu \mathrm{M} \mathrm{Pb}^{2+}$ for $2 \mathrm{~h}$; (E) after regeneration of channel with fresh substrate DNA; and (F) $\mathrm{Pb}^{2+}$ detection in the regenerated channel.

Figure 68. Fluorescence images of PMMA surface under the PDMS channel: Immobilized enzyme DNA spot where the channel is clearly observed passing through the spot (A); the spot after hybridization with substrate DNA tagged with quencher (B); and $\mathrm{Pb}^{2+}$ flow through the channel cleaves the substrate and results in a fluorescence increase (C).

Figure 69. Scheme for immobilization of DNAzyme on glass microbeads.

Figure 70. Fluorescence images of glass microbeads before and after DNA immobilization.

Figure 71. Steps involved in creating mold for constricted channel.

Figure 72. Side view of PDMS device with constricted channel.

Figure 73. Fluorescence images after incorporation of beads in the PDMS channel, hybridization, and $\mathrm{Pb}$ reaction.

Figure 74. Scheme for immobilization of DNAzyme on glass microbeads.

Figure 75. Fluorescence images of: glass microbeads after DNA immobilization (A), hybridization (B), and exposure to $10 \mu \mathrm{M} \mathrm{Pb}^{2+}(\mathrm{C})$.

Figure 76. Fluorescence images after: incorporation of beads in the PDMS channel (A), hybridization (B), exposure to $10 \mu \mathrm{M} \mathrm{Pb}^{2+}(\mathrm{C})$, and $100 \mu \mathrm{M} \mathrm{Pb}^{2+}(\mathrm{D})$.

Figure 77. Baseline-corrected ferrocenyl anodic peaks from cyclic voltammograms scanned from $0.0 \mathrm{~V}$ to $0.6 \mathrm{~V}$ showing $\mathrm{Pb}^{2+}$-DNAzyme activity. After $10 \mathrm{~min}$ of reaction with $10 \mu \mathrm{M} \mathrm{Pb}^{2+}$, signal loss is $~ 80 \%$ compared to less than $10 \%$ in the sample that has no $\mathrm{Pb}^{2+}$.

Figure 78. From top left to bottom right: immobilization of DNAzyme and subsequent $\mathrm{Pb}^{2+}$ detection on fluorous-coated silica packed in a PDMS channel. ...

Figure 79. Fluorescence spectra of eluent from channel after exposure to $\mathrm{Pb}^{2+}$..

Figure 80. (A) Structure of the $\mathrm{UO}_{2}{ }^{2+}$ specific DNAzyme showing the top substrate strand and the bottom enzyme strand. (B) The lead-specific DNAzyme structure for comparison. (C) The fluorosensing scheme. (D) The fluorescent response of the $\mathrm{UO}_{2}{ }^{2+}$ specific DNAzyme after $10 \mathrm{~min}$. In the absence of $\mathrm{UO}_{2}{ }^{2+}$, fluorescence at $525 \mathrm{~nm}$ remains completely quenched. In the presence of $\mathrm{UO}_{2}{ }^{2+}$, the fluorescein label is visible. (E) The response of the substrate DNAzyme without the enzyme (lane 1), and the response of the

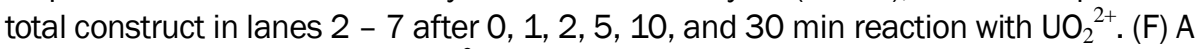
sensor array containing both $\mathrm{UO}_{2}{ }^{2+}$ and $\mathrm{Pb}$ sensors demonstrating the potential for multiplexing.

Figure 81. Fluorescence images showing spots with various combinations of enzyme and substrate DNA and metal ions.

Figure 82. Device design for multianalyte (lead and uranium) analysis on a single injection. 


\section{Tables}

Table 1. Certified metal content in electroplating sludge reference material.....................................15

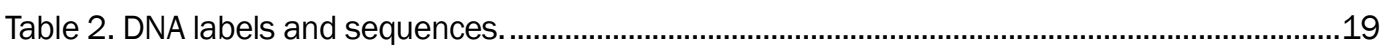

Table 3. Electroosmotic coefficients $\left\{\mathrm{cm}^{2} / \mathrm{V} \cdot \mathrm{s}\right\}$ for phosphate buffer solution versus $\mathrm{pH}$ measured using the current monitoring method.....................................................................................2

Table 4. Analytes used for testing. ...............................................................................................46 


\section{Preface}

This study was conducted for the Strategic Environmental Research and Development Program (SERDP) under Project ER-1459, "Metal Ion Sensor with Catalytic DNA in a Nanofluidic Intelligent Processor." The SERDP technical monitor was Dr. Andrea Leeson, Program Manager for Environmental Restoration.

The work was performed by the Environmental Processes Branch (CN-E) of the Installations Division (CN), U.S. Army Engineer Research and Development Center - Construction Engineering Research Laboratory (ERDC-CERL). At the time of publication, Debbie Curtin was Chief, CEERD-CN-N; J ohn Bandy was Chief, CEERD-CN; and Alan Anderson was the Technical Director for Environmental Quality. The Deputy Director of ERDC-CERL was Dr. Kirankumar Topudurti and the Director was Dr. Ilker Adiguzel.

COL Kevin J. Wilson was the Commander and Executive Director of ERDC, and Dr. J effery P. Holland was the Director. 


\section{Acknowledgements}

Several individuals and organizations participated in this project and provided valuable review, guidance, information, access to facilities, and logistical support. The authors acknowledge the following persons and organizations for their primary participation:

- Beckman Institute of Advanced Science and Technology, Urbana, IL

- University of Illinois at Urbana-Champaign, IL: Department of Chemistry and Department of Mechanical Science and Engineering

- University of Notre Dame, South Bend, IN: Department of Chemical and Biomolecular Engineering and Department of Chemistry and Biochemistry

- U.S. Army Corps of Engineers, Engineer Research and Development Center - Construction Engineering Research Laboratory (ERDC-CERL) at Champaign, IL

- Dr. Xiaofeng Wang

- Ms. Bethany Reynolds

The authors also acknowledge and appreciate the assistance of Dr. Bruce Flachsbart, B.Y. Kim, J . Yang, M. Gong, and D.P. Wernette, all from the University of Illinois, for their assistance with various aspects of chip fabrication, microfluidic separations, and DNA hybridization experiments. The authors also thank S. Prakash, A. Piruska, E.N. Gatimu, S.P. Branagan, A.B. Minnis, Z. Wang, and T.L. King, all from the University of Notre Dame, for their additional research in surface metallization, device fabrication, membrane transport experiments, and computational modeling of electric fields. 


\section{Unit Conversion Factors}

\begin{tabular}{|c|c|c|}
\hline Multiply & By & To Obtain \\
\hline angstroms & 0.1 & nanometers \\
\hline atmosphere (standard) & 101.325 & kilopascals \\
\hline bars & 100 & kilopascals \\
\hline British thermal units (International Table) & $1,055.056$ & joules \\
\hline centipoises & 0.001 & pascal seconds \\
\hline centistokes & 1.0 E-06 & square meters per second \\
\hline cubic feet & 0.02831685 & cubic meters \\
\hline cubic inches & $1.6387064 \mathrm{E}-05$ & cubic meters \\
\hline cubic yards & 0.7645549 & cubic meters \\
\hline degrees (angle) & 0.01745329 & radians \\
\hline degrees Fahrenheit & $(\mathrm{F}-32) / 1.8$ & degrees Celsius \\
\hline feet & 0.3048 & meters \\
\hline foot-pounds force & 1.355818 & joules \\
\hline gallons (U.S. liquid) & $3.785412 \mathrm{E}-03$ & cubic meters \\
\hline inches & 0.0254 & meters \\
\hline inch-pounds (force) & 0.1129848 & newton meters \\
\hline kilotons (nuclear equivalent of TNT) & 4.184 & terajoules \\
\hline knots & 0.5144444 & meters per second \\
\hline microinches & 0.0254 & micrometers \\
\hline microns & $1.0 \mathrm{E}-06$ & meters \\
\hline miles (nautical) & 1,852 & meters \\
\hline miles (U.S. statute) & $1,609.347$ & meters \\
\hline miles per hour & 0.44704 & meters per second \\
\hline mils & 0.0254 & millimeters \\
\hline ounces (mass) & 0.02834952 & kilograms \\
\hline ounces (U.S. fluid) & 2.957353 E-05 & cubic meters \\
\hline pints (U.S. liquid) & 4.73176 E-04 & cubic meters \\
\hline pints (U.S. liquid) & 0.473176 & liters \\
\hline pounds (force) & 4.448222 & newtons \\
\hline
\end{tabular}




\section{Introduction}

\subsection{Background}

Heavy metals are a ubiquitous and troublesome class of pollutants. Among heavy metals, lead occupies a prominent position as a contaminant requiring constant attention (CDC 1991). Non-biodegradable lead is known to accumulate in the environment and produce numerous toxicological effects, especially in children. The high profile of lead is linked both to its numerous toxicological effects and the wide range of its potential exposure, including being present in air, dust, soil, and water.

Origins of lead in the natural environment result from both historical and current uses of lead compounds. Runoff along the drip line of buildings, some dating from as far back as World War II, still contain lead derived from the lead-based paints commonly used at the time. Outdoor metal structures, such as bridges, were frequently covered in red and white lead primers, from which lead is released during subsequent weathering and refurbishing. Some industrial activities, such as the smelting of lead acid batteries, produce a waste stream high in lead.

Anthropogenic sources of lead from military operations require active monitoring to ensure environmental compliance and protection. Despite the recognized adverse effects of lead on aquatic and land-based biota, its presence is not actively monitored in the field. Current testing involves sample collection followed by complex laboratory testing. Specifically, there is no available, portable product that meets all requirements for in situ measurement of lead in ground water (i.e., rugged, reliable, sensitive, selective, and remotely operable).

\subsection{Objective}

Lead is a primary contaminant of concern in the heavy metal class of environmental pollutants. Public laws and agency directives require vigilance for lead in air, dust, soil, and water. Subsequently, the Department of Defense requires vigilant monitoring of firing ranges because of leadcontaining munitions. 
Our working hypothesis was that catalytic DNAzymes can be immobilized within "lab on a chip" constructs to remain active, selective, and sensitive toward target metal analytes. We then combined this sensing molecule with chromatographic methods and a detection platform to enhance performance.

Specific objectives of this research were:

- Create a highly selective and sensitive miniature sensor for lead by combining two recent advances: (a) catalytic DNA that is reactive only to lead and which can be tagged to produce fluorescence only in the presence of the metal, and (b) nanoscale fluidic molecular gates that can manipulate fluid flows and perform molecular separations on tiny volumes of material.

- Further develop the chemistry and engineering needed to create a microfluidic device for separating, sensing, and quantifying lead in a complex matrix.

- Manipulate the sensor platform for separation and detection of other heavy metals to target not only the development of a specific sensor for $\mathrm{Pb}^{2+}$ but also the development of a class of highly specific sensors for other analytes.

- Demonstrate sensor performance on real-world samples.

\subsection{Approach}

This study's hypothesis was that catalytic DNA can be immobilized within "lab on a chip" constructs to remain active, selective, and sensitive toward target metal analytes. Capillary electro-phoresis columns were microfabricated in polydimethylsiloxane and polycarbonate to precisely control fluidic movement by applying an electric field across distal ends of the columns. A three-dimensional arrangement of channels provide the option to pass a selectable sample volume through a novel molecular gate polymeric membrane perforated with a number of long nanometer-width channels. Specific recognition elements that cause a measurable response in the presence of a particular analyte can be incorporated in channels or microchannels on the opposing side of the molecular gate membrane. In either case, the device's detection zone was chemically modified with a unique sequence of catalytic DNA that cleaves an associated strand of substrate DNA in the presence of lead. Tagging the substrate DNA with a fluorophore allows for detection of the substrate DNA fragments, thus providing a sensitive optical signal for the presence of lead. This research was concerned with all chemical aspects of design and function for a rug- 
ged miniaturized sensor that is capable of remote, selective, and sensitive detection of bioavailable lead.

\subsection{Scope}

The design was targeted at waterborne lead as one of the Army's top ten emerging contaminants. This work successfully demonstrated the feasibility of incorporating $\mathrm{Pb}_{2}^{+}$-specific catalytic DNA in a polymer-based microfluidic device to detect $\mathrm{Pb} 2^{+}$. The work also uncovered related advances such as creating microfluidic devices in PMMA.

\subsection{Mode of Technology Transfer}

Much of the project's output has been reported through peer-reviewed journals and prominent conferences. This report also will be made accessible through the World Wide Web (WWW) at URL: http:// www.cecer.army.mil. 


\section{Regulatory Drivers}

Lead is a hazardous metal according to the Resource Conservation \& Recovery Act (RCRA), and it is also a U.S. Environmental Protection Agency (EPA) urban air toxic as designated in the 1990 amendment to the Clean Air Act. Various rulings and standards on lead in the environment have come from such federal acts as 40 CFR Part 745 wherein the EPA has identified dangerous levels of lead, based on Section 403 of the Toxic Substances Control Act (TSCA).

In addition, numerous military directives have been issued for the management of toxic substances such as lead, including Army Regulation 2001, "Environmental Protection and Enhancement." Of added importance to the military is guidance from Department of Defense (DoD) Directive 4715 that requires a high degree of management and monitoring of firing ranges to maintain operational readiness while protecting human health and the environment. The impact areas on these firing ranges are replete with lead due to use of numerous lead containing munitions. Range managers must carefully monitor leaching of lead from the berms to protect downstream habitats. These examples and others would benefit immensely from a field-portable lead sensor designed and equipped with features and characteristics that would allow real time, in situ measurement of lead in ground water.

In addition, SERDP has established a long-term ecosystem management research program at Fort Benning, Georgia, known as SEMP. A major goal of this program is to develop indicators of ecosystem health as well as indicators of ecosystem change. As part of SEMP, the Environmental Characterization and Monitoring Initiative (ECMI) monitors numerous meteorological, soil, and water conditions as well as plant and animal presence.

This ecosystem monitoring program is focused on maintaining and improving the sustainability and native biological diversity on military lands while supporting human needs, including the DoD mission. This program establishes long term monitoring sites on DoD lands to monitor the nonsteady state of ecosystems, especially the effects of military activities. Current efforts include measuring equipment and remote sensors for acquisition of water quality field data. Critical parameters such as heavy metal 
concentrations are not being measured, however, due to the lack of a field product that meets all requirements for remote metal sensing. While lead is of primary interest, we believe other metal contaminants such as mercury, cobalt, arsenic, and uranium could also be detected in groundwater merely by changing the catalytic DNA on the sensor chip discussed here.

\subsection{Summary of past research}

\subsubsection{Catalytic DNA}

In 1994, DNA was shown through a technique called in vitro selection (vide infra) to carry out catalytic functions when single stranded (Breaker and J oyce 1994). The DNAs (called catalytic DNA or DNA enzymes or deoxyribozymes) have proven capable of catalyzing many reactions including RNA/ DNA-cleavage, ligation, phosphorylation, cleavage of phosphoramidate bonds, and porphyrin metallation (Breaker 1997).

In 2000, Li and Lu reported a new application for catalytic DNAs as biosensors for metal ions (specifically $\mathrm{Pb}^{2+}$ ) by showing the DNAzymes would selectively react with $\mathrm{Pb}^{2+}$ in the presence of interfering cations ( $\mathrm{Li}$ and $\mathrm{Lu}$ 2000). This application is based on the observation that catalytic DNA, obtained through in vitro selection, can be used to bind metal ions with high affinity and specificity (Breaker and J oyce 1994; Li et al. 2000; Santoro et al. 2000). Therefore, the activity of a selected catalytic DNA can be used to measure the identity and quantity of the specific metal ion.

The biosensor consists of a catalytic DNA capable of base-pairing to a DNA substrate containing a single ribonucleotide residue (labeled rA in Figure 1A). When a fluorophore, e.g., carboxytetramethylrhodamine (TAMRA), is attached to the 5 ' end of the DNA substrate, the fluorescence signal at 580 $\mathrm{nm}$ is quenched by its proximity to a fluorescence quencher, e.g. 4-((4dimethylamino)-phenyl)azo)benzoic acid (Dabcyl), at the nearby 3'-end of the catalytic DNA. In the presence of $\mathrm{Pb}^{2+}$, the fluorescence emission of TAMRA increases dramatically ( $400 \%$ ) due to the cleavage of the substrate DNA and subsequent separation of the fluorophore from the quencher. This is followed by the release of substrate DNA fragments and $\mathrm{Pb}^{2+}$ (Figure 1B). 


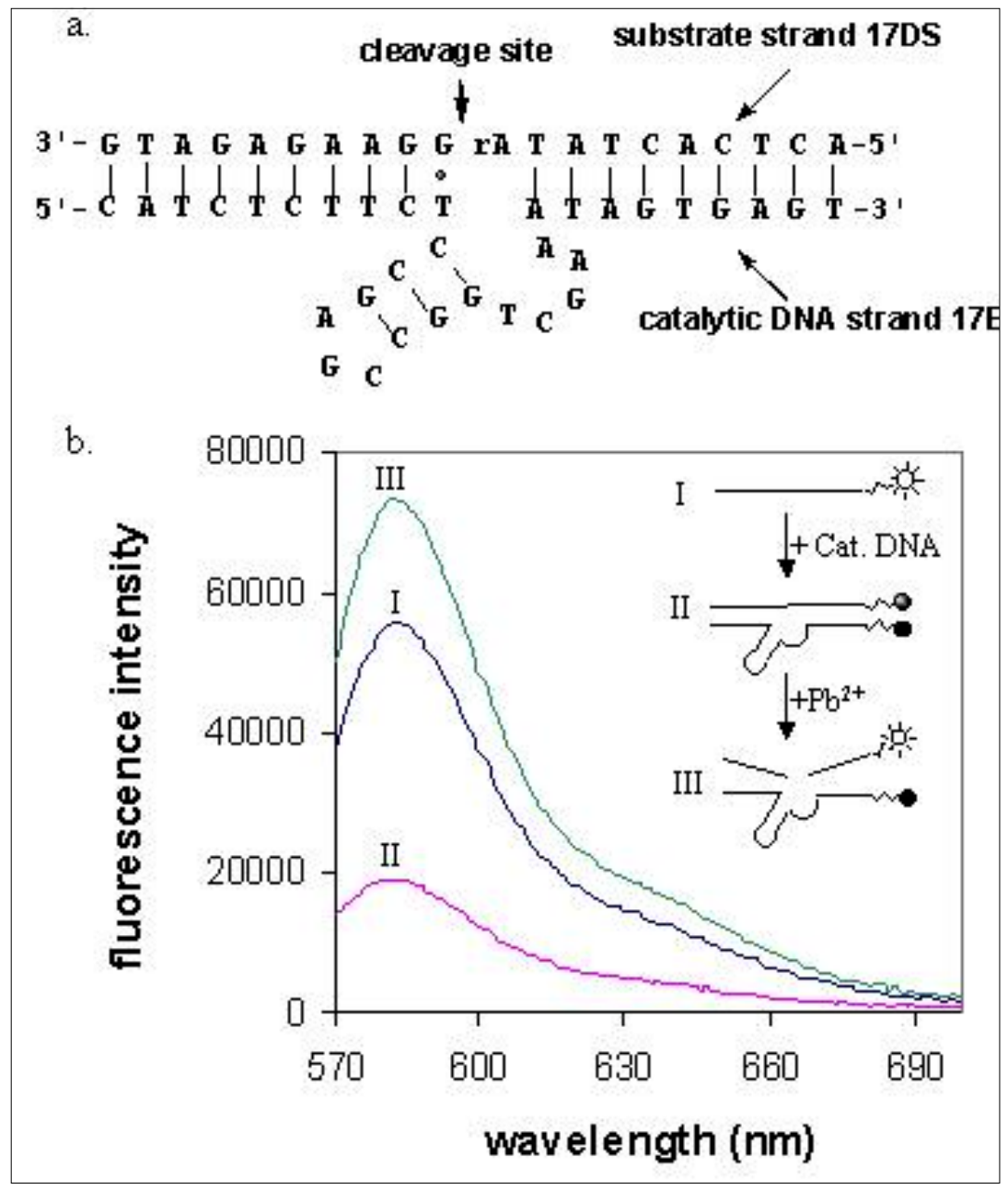

Figure 1. (A) Structure of the Pb-sensing catalytic DNA and (B) fluorosensing mechanism and results.

This system represents a new class of metal ion sensors and is the first example of using the powerful tools of combinatorial molecular biology to identify a cation-specific catalytic DNA for sensing of metal ions. It combines the high selectivity of catalytic DNA with the ultralow background and resulting high sensitivity of fluorescence detection, and it can be applied to quantitative detection of $\mathrm{Pb}^{2+}$ over a concentration range of three orders of magnitude. The sensitivity and selectivity of the system can be altered by using different fluorescence/ quencher pairs for different sensors.

The system is easily regenerated by washing away the cleaved products and adding new substrate DNA to the catalytic DNA strand - a fact that will be exploited to regenerate active material in the proposed sensor (Swearingen et al. 2005). Finally, catalytic DNA specific for other metal 
ions and with various detection ranges can be isolated by varying the catalytic DNA selection conditions in the combinatorial search, making this approach extensible to other metal ions.

\subsubsection{Metal ion sensors}

Metal ions play important roles in biological systems. Beneficial metal ions such as $\mathrm{Ca}^{2+}, \mathrm{Fe}^{3+}$ and $\mathrm{Mg}^{2+}$ are minerals required to maintain normal functions, while toxic metal ions such as $\mathrm{Pb}^{2+}, \mathrm{Hg}^{2+}$ and $\mathrm{Cr}^{6+}$ can have a number of adverse health effects. Current methods for metal ion determinations, such as atomic absorption spectrometry (Parsons and Slavin 1993), inductively coupled plasma mass spectrometry (Aggawal, Kinter, and Herold 1994), and anodic stripping voltammetry (J agner, Renman, and Wang 1994) often require sophisticated equipment or sample treatment, which makes them unsuitable for field monitoring due to size, power requirements, and fragility. Simple and inexpensive methods that permit real-time, on-site sampling of metal ions would constitute a critical enabling advance in the field.

Fluorosensors based on fluorescently-labeled organic chelators, proteins, or peptides have emerged as powerful tools toward achieving the above goals (Tsien 1993; Czarnik 1995; Oehme and Wolfbeis 1997). While remarkable progress has been made in developing fluorosensors for metal ions such as $\mathrm{Ca}^{2+}$ and $\mathrm{Zn}^{2+}$, designing and synthesizing sensitive and selective metal ion fluorosensors remains a significant challenge. Perhaps the biggest challenge in fluorosensor research is the design and synthesis of a sensor capable of specific and strong metal-binding. Since our knowledge about the construction of metal-binding sites is limited, searching for sensors in a combinatorial way can drastically reduce the research effort required to identify effective fluorescently-active chelating agents. In this regard, in vitro selection of DNA/RNA from a library of $10^{14}-10^{15}$ random DNA/ RNA sequences offers considerable opportunity (Breaker and J oyce 1994; Breaker 1997). Compared with combinatorial searches of chemoand peptidyl-sensors, in vitro selection of DNA/ RNA is capable of sampling a larger pool of sequences, amplifying the desired sequences by the polymerase chain reaction (PCR), and introducing mutations to improve performance by mutagenic PCR. For example, the in vitro selection method has been used to obtain DNA/ RNA aptamers (Potyrailo et al. 1998; J haveri et al. 2000) and aptazymes (Robertson and Ellington 1999; Koizumi et al. 1999) that are responsive to small organic molecules. Similarly, 
catalytic DNA/ RNAs that are highly specific for $\mathrm{Pb}^{2+}$ (Breaker and J oyce 1994; Pan and Uhlenbeck 1992), $\mathrm{Ca}^{2+}$ (Cuenod and Szostak 1995) and $\mathrm{Zn}^{2+}$ (Li, Kwon and Lu 2000; Santoro et al. 2000) have been obtained. These results set the stage for the utilization of catalytic DNAs with hydrolytic cleavage activity for detection of metal ions.

\subsubsection{Microfabricated analysis systems}

A trend throughout the last two decades has been towards miniaturized analytical methods to achieve unique capabilities as well as better performance specifications. The development of microelectro-mechanical systems (MEMS) and the related concept of miniaturizing a total analytical system, $\mu$-TAS, started with the work of Terry et al. (1979), who fabricated a complete gas chromatography system on a silicon wafer. Manz and coworkers reached another milestone with liquid phase separations (Manz, Graber and Widmer 1990; Manz et al. 1992), followed rapidly by pioneering work by Harrison and Manz (Harrison et al. 1993), Ramsay (J acobson et al. 1994), and Mathies (Woolley and Mathies 1994). Since then, there has been an exponential growth in this field, including advances in fabrication methods from silicon (Christel et al. 1998), optically transparent materials (Ruano et al. 1999; Backhouse et al. 2000), and exciting advances in polymer substrates (Folch et al. 1999; McDonald et al. 2000). Besides analytical microfluidics, a tremendous effort has gone into the development of mixers, valves, interconnects, filters, and other elements required for a complete microfluidics system (Koch et al. 1999). Recent reviews describe the important milestones in this research area (Kricka 1998).

\subsubsection{Fluidic control with molecular gates}

The Bohn Research Group has developed a new approach to fluidic control, in which a "molecular gate" is constructed from a thin (typically $5 \mu \mathrm{m}$ ) polymeric membrane perforated with a number $\left(\sim 10^{8} \mathrm{~cm}^{-2}\right)$ of long, narrow (typical aspect ratios of 25-250) channels (Kemery, Steehler, and Bohn 1998). These structures exhibit unique and tunable electrokinetic flow properties because the product of the channel diameter, a, and the inverse Debye length, $\kappa$, is $\sim 1$. When $\kappa$ a $<1$, the electric double layer extends throughout the pore (Saksena and Zydeney 1995; Basu \& Sharma 1997; Kim and Stevens 1997), and the mobile counterions filling the channel determine transport. At the other limit, $\kappa$ a $>1$, the electrical double layer is mostly collapsed, and normal ion migration effects dominate. 
Thus, flow can be controlled by (a) direction of the applied bias, (b) sign and surface density of the immobile charge on the wall, (i.e., $\mathrm{pH}$ and chemical derivatization), and (c) the magnitude of $\kappa$. Because $\kappa$ is controlled by solution ionic strength, control of flow in these nanometer channels is exceptionally versatile. These nanoscale, porous materials with well-defined cross-sectional geometry are ideally suited to making intelligent interconnects between microfluidic elements. This occurs because the interconnect itself can be made integral to the intelligent movement of biomolecules, and it is simple to integrate and interface with existing microfluidic technologies. Combining catalytic DNA with molecular gates, to achieve a nanofluidic intelligent processor (NIP), made it possible to build a sensitive, selective $\mathrm{Pb}^{2+}$ sensor (Chang et al. 2005), as shown in Figure 2.

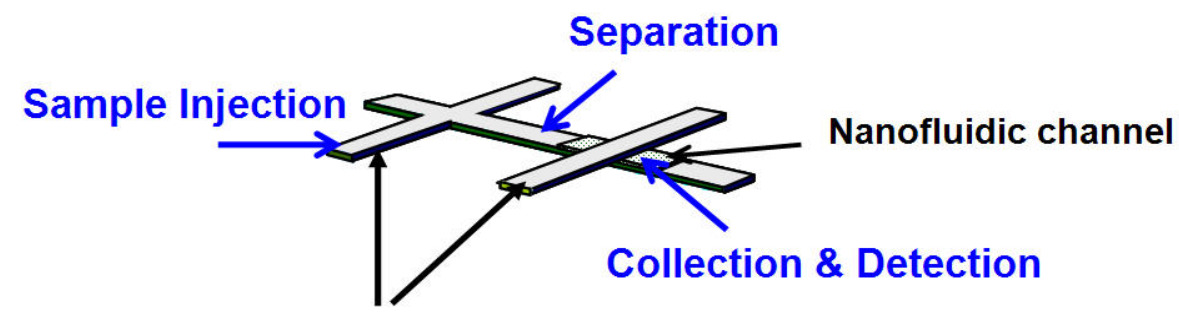

PDMS microfluidic channels

$50 \mu \mathrm{m}^{2}$ channel dimensions

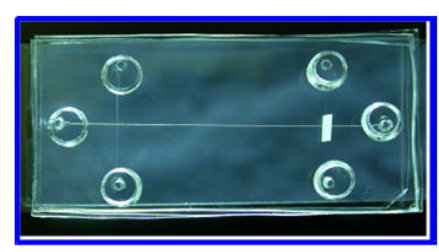

PDMS device on microscope slide

Figure 2. Structure of the microfluidic device for heavy metal detection.

\subsubsection{Miniaturized microfluidic lead sensor}

As proof of principle for this SERDP project, our previous SEED proposal developed and demonstrated a miniaturized lead sensor by combining a lead-specific DNAzyme with a microfabricated device containing a network of microfluidic channels that are fluidically coupled via nanocapillary array interconnects. The DNAzyme construct (Figure 1) was derivatized with fluorophore (quencher) at the $5^{\prime}\left(3^{\prime}\right)$ end of the substrate and enzyme strand, respectively, to form the molecular beacon that is used as the recognition element. The nanocapillary array membrane interconnect is used to manipulate fluid flows and deliver the small volume sample to the beacon in a spatially confined detection window where the DNAzyme is interrogated using laser-induced fluorescence (LIF) detection. 
We had already studied the function of the lead-specific DNAzyme in a previous report. The substrate cleavage reaction was monitored using fluorescence spectroscopy $\left(\lambda_{\mathrm{ex}}=560 \mathrm{~nm}, \lambda_{\mathrm{em}}=580 \mathrm{~nm}\right)$ that illustrated its excellent sensitivity, dynamic range (quantifiable detection in the range of 10 $\mathrm{nM}<\left[\mathrm{Pb}^{2+}\right]<10 \mu \mathrm{M}$ ), and selectivity (at least an 80-fold selectivity enhancement over other divalent metals) for $\mathrm{Pb}^{2+}$ ( $\mathrm{Li}$ and $\mathrm{Lu} 2000$ ). As shown in Figure 3, the resultant fluorescent signal in response to the presence of lead is significantly greater than other common divalent cationic species.

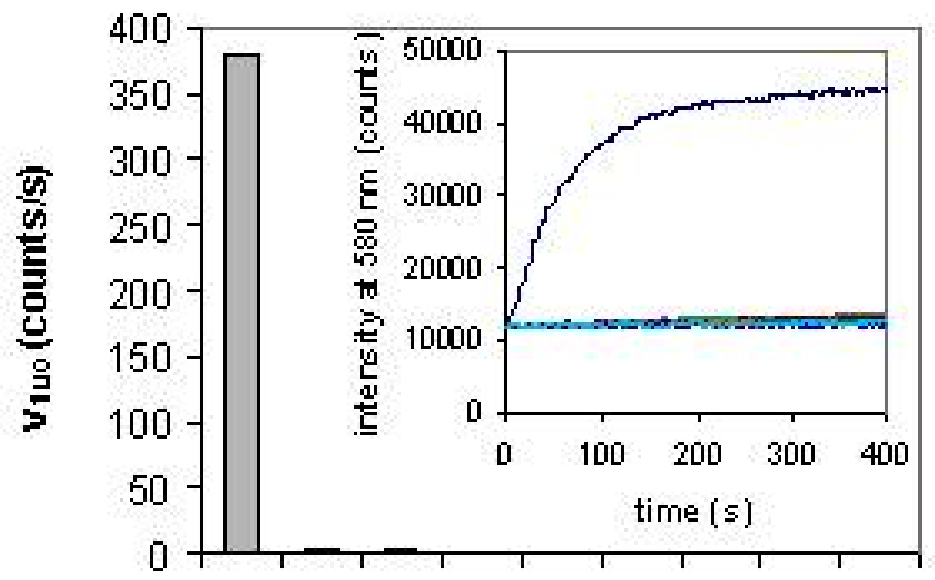

Pb Co Zn Mn Ni Cd Cu Mg Ca

Figure 3. DNAzyme selectivity compared to common divalent cationic species.

We fabricated a simple two-channel device (Figure 4) with a source channel for introduction of the sample and a receiving channel containing the hybridized DNA, separated by the nanocapillary array interface (NAI). The fluid flow is controlled by electrical-bias pathways for injection of the sample from the source channel across the NAI into the receiving channel. 


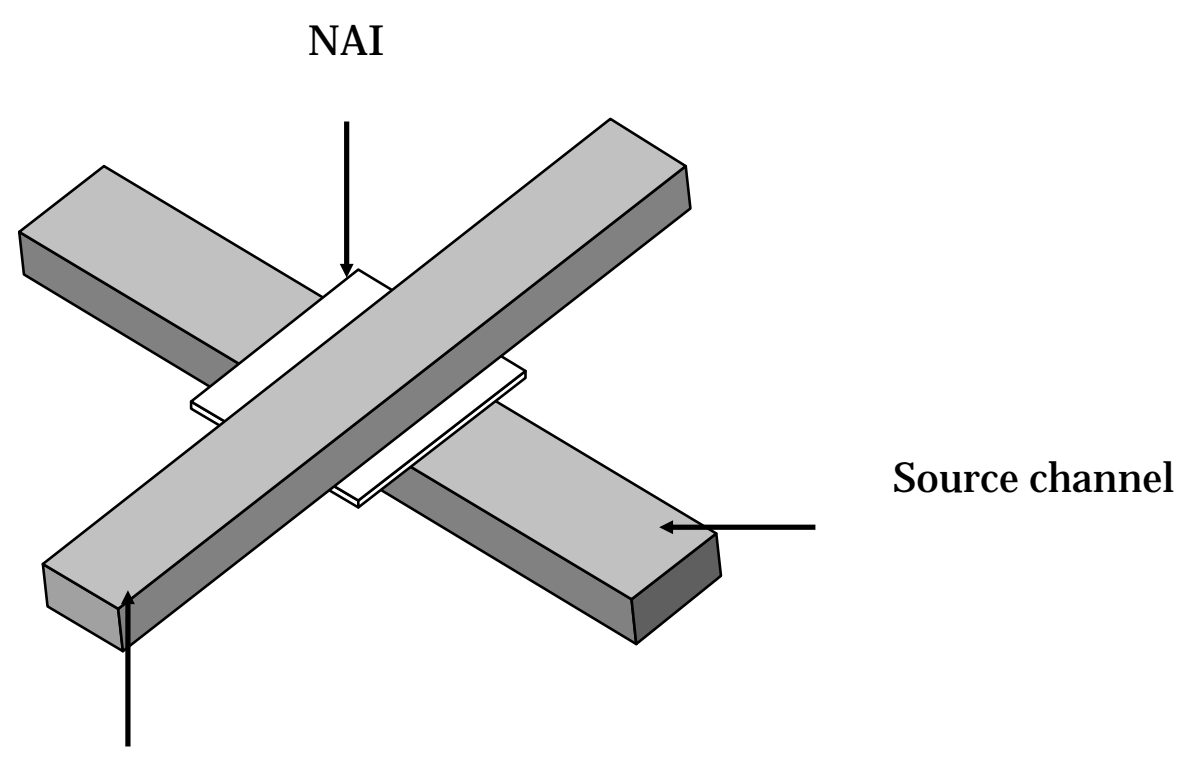

Receiving channel

Figure 4. Schematic of a three-dimensional NAl gateable microfluidic device. Both crossed-microfluidic channels are identical with dimensions of $50-\mu \mathrm{m}$ width, 30- $\mu \mathrm{m}$ depth, and 14-mm length.

The sequential images in Figure 5A- Figure 5F capture the fluorescence from repeated injections of $\mathrm{Pb}^{2+}$ at the intersection of the cross-channels. Figure 5A shows the background fluorescence of the two-channel system. The dotted white lines indicate the position of the horizontal channel and fluorescence from the vertical channel is barely observable. In the on state, $\mathrm{Pb}^{2+-}$ containing solution is electrostatically driven from the horizontal channel across the NAI to the vertical channel containing the DNAzyme (Figure 5B- Figure 5C).

As soon as the on-state bias is applied (Figure 5B), the $\mathrm{Pb}^{2+}$ plug starts to cleave the DNAzyme in the receiving channel, resulting in a significant fluorescence increase. The off state switches the positive bias on the source channel to ground, stopping the injection of $\mathrm{Pb}^{2+}$ into the receiving channel and flushing the cleaved DNA strand to the upper reservoir (Figure 5D) until all cleaved DNA is removed from the viewing image (Figure 5E). Repeated injections of $\mathrm{Pb}^{2+}$ solution (Figure 5F) showed that this microfluidic chip can be used repeatedly. 

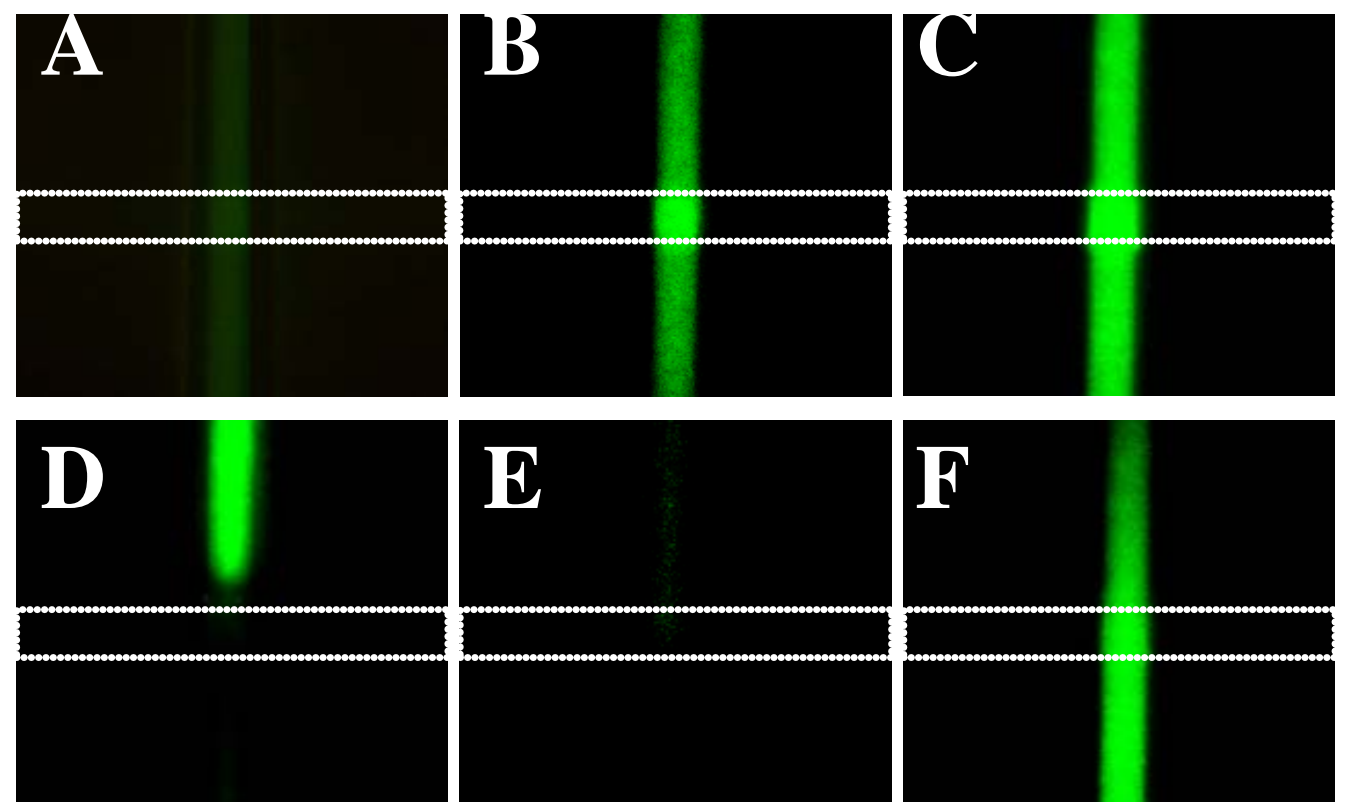

Figure 5. Temporal sequence of fluorescence images at the intersection of the crossed microchannels. Source channel (horizontal) was filled with $1 \mu \mathrm{M} \mathrm{Pb}^{2+}$ in BGE ( $25 \mathrm{mM}$ lactic acid, $25 \mathrm{mM} \mathrm{HEPES}, 50 \mathrm{mM} \mathrm{NaCl}$ ) and receiving channel (vertical) was filled with hybridized DNAzyme in BGE. Dashed lines indicate the position of the horizontal source channel. In the off-state (A), all reservoirs were floated while the on-state (B), causes injection of $\mathrm{Pb}^{2+}$ solution. Lead solution is transferred from the source channel, across the NAl toward grounded reservoir. The reaction with DNAzyme produced fluorescence from cleaved DNA in the receiving channel (C). During on state bias, the DNA cleavage reaction reached equilibrium and a constant fluorescent signal is maintained (captured image is $\sim 40 \mathrm{~s}$ after on-state bias is applied). $\mathrm{Pb}^{2+}$ plug continued to move to the ground electrode and cleaved DNA moved toward the positive bias. Image (D) is $\sim 1 \mathrm{~s}$ after off state bias is applied and (E) is $\sim 40 \mathrm{~s}$ after off state bias is applied. Cleaved DNA moved toward the positive bias and the receiving channel was flushed with bulk hybridized DNAzyme solution. Repetitive $\mathrm{Pb}^{2+}$ plug injection after switching back to the on-state bias is shown in (F).

Calibration of this DNA biosensor-coupled microfluidic system was accomplished by measuring fluorescence intensity (DNAzyme substrate strand cleavage efficiency) as a function of $\mathrm{Pb}^{2+}$ concentration in the range of $100 \mathrm{nM}<\left[\mathrm{Pb}^{2+}\right]<200 \mu \mathrm{M}$. A calibration plot of fluorescence enhancement vs. lead concentration is shown in Figure 6. At each measurement, electrical bias was cycled four times between on and off states. Each datum in the plot represents the average fluorescence enhancement of these four trials as a function of $\mathrm{Pb}^{2+}$ concentration and the error bars represent $\pm \sigma$. The expression of best fit for the plot is described as $\ln (\operatorname{Imax}-\mathrm{I})=$ $\square 0.0436\left[\mathrm{~Pb}^{2+}\right]+4.2072$, where Imax and I are maximum fluorescence enhancement (\%) and fluorescence enhancement (\%) at the lead concentration, respectively. The microfluidic system response has a linear corre- 
lation using the above expression over the $100 \mathrm{nM}$ to $200 \mu \mathrm{M}$ concentration range with a correlation coefficient $\left(\mathrm{r}^{2}\right)$ of 0.98 . This range likely encompasses $\mathrm{Pb}^{2+}$ concentration levels for most environmental samples.

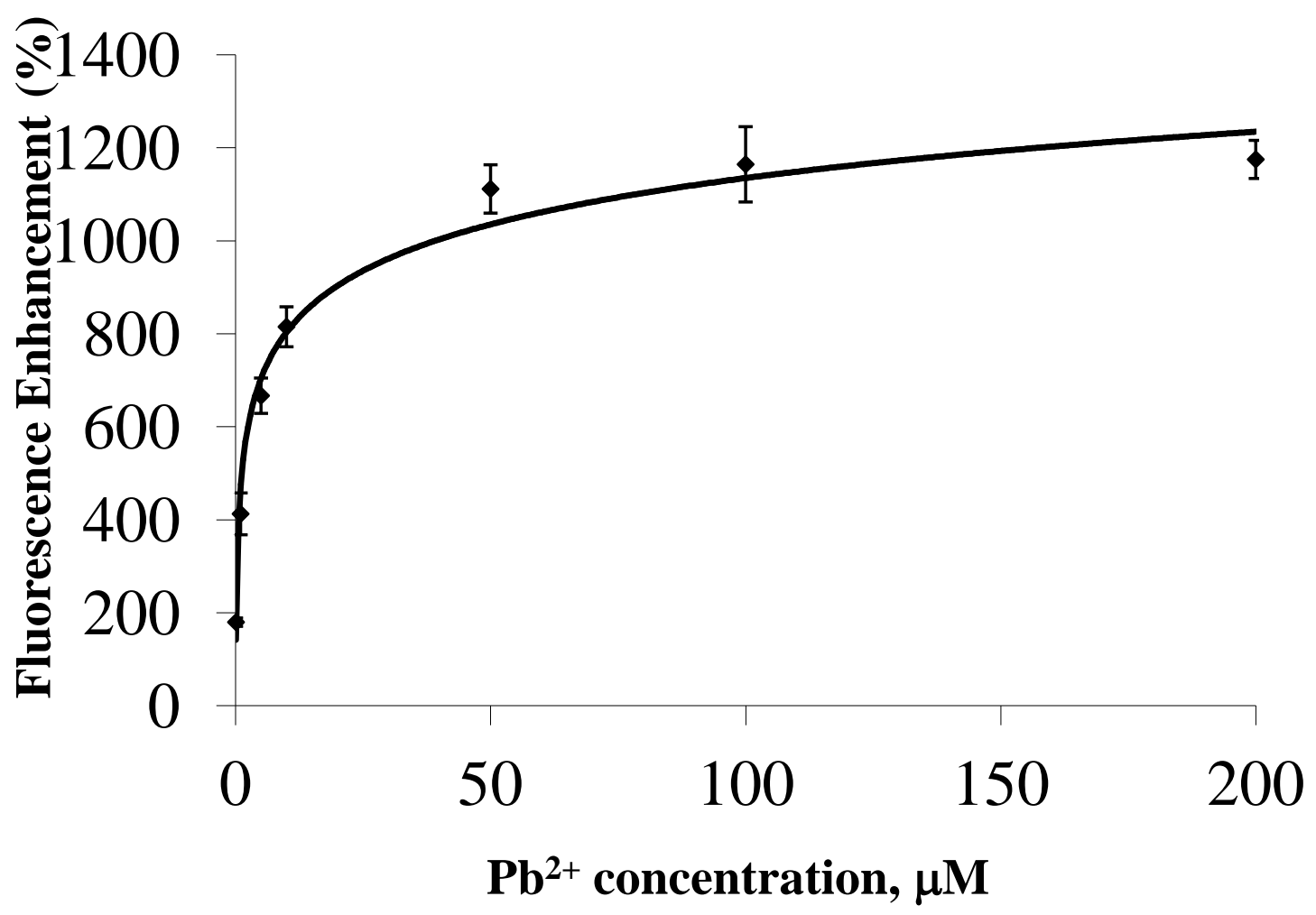

Figure 6. Plot of fluorescence enhancement as a function of lead ion concentration from 0.1, $1,5,10,50,100$, and $200 \mu \mathrm{M}$. Error bars represent $\pm 1 \sigma(n=4)$.

Repetitive detection of $100 \mathrm{nM} \mathrm{Pb} 2+$ is illustrated in Figure 7. During repetitive injection sequences, signals were reproducible with a coefficient of variation of 3.5\% $(n=5)$ and the baseline consistently returned to a constant level. The detection limit was evaluated by repetitive injection of 50 $\mathrm{nM} \mathrm{Pb}^{2+}$ standard solution. From the baseline noise during the off state and the fluorescence intensity of $50 \mathrm{nM} \mathrm{Pb}^{2+}$ during the on state, the detection limit (signal-to-noise ratio of 3:1) was determined to be $11 \mathrm{nM}(2.2$ $\mathrm{ppb}$ ), which is lower than the $72 \mathrm{nM}$ ( $15 \mathrm{ppb}$ ) action level in drinking water recommended by the USEPA. These results demonstrate that the combination of electrokinetically actuated measurement cycles on a microfluidic device and a $\mathrm{Pb}^{2+}$-selective DNAzyme produce a device sensitive enough to monitor lead in drinking water or ground water. 


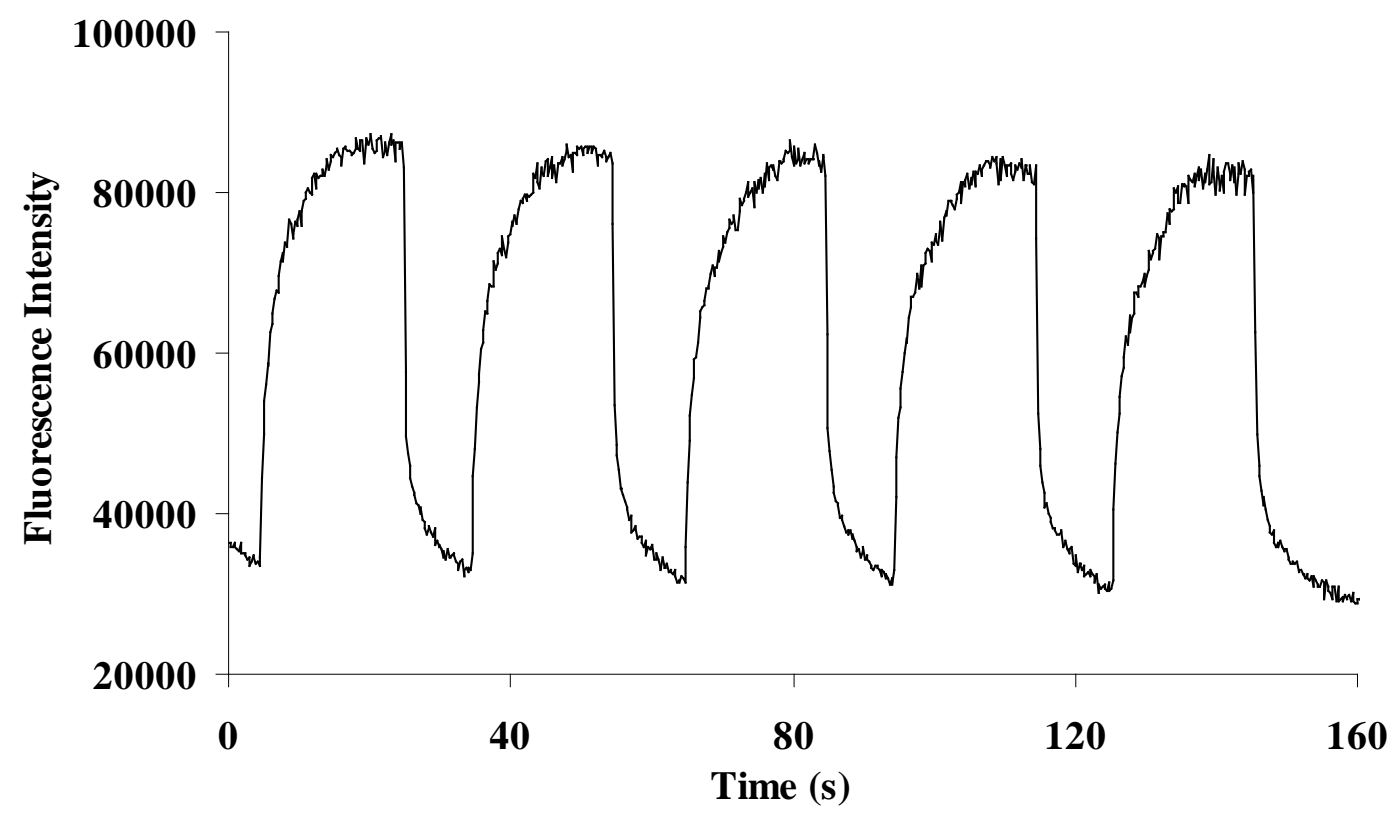

Figure 7. Typical fluorescence signals during repetitive $\mathrm{Pb}^{2+}$ injection (100 nM in BGE). Detection was performed at the intersection of crossed channels. On state was maintained for $20 \mathrm{~s}$ and switched to off state for $10 \mathrm{~s}$, repetitively.

We challenged this microfluidic DNAzyme sensor against a complex matrix, determination of $\mathrm{Pb}^{2+}$ content in an electroplating sludge standard reference material. The electroplating sludge sample was purchased from Resource Technology Corporation (Laramie, WY) and prepared for analysis by USEPA Method 3050B. The sludge sample was thoroughly mixed, dried and ground immediately before use. For the digestion, $2.5 \mathrm{~mL}$ of concentrated $\mathrm{HNO} 3$ and $10 \mathrm{~mL}$ of concentrated $\mathrm{HCl}$ were added to $0.1 \mathrm{~g}$ sample and refluxed for $15 \mathrm{~min}$ on a hot plate. The digestate was filtered through Whatman No. 41 filter paper, and the filtrate was collected in a volumetric flask. The filter paper and the residue were both washed with 5 $\mathrm{mL}$ of hot $\mathrm{HCl}$. These washings were collected in the same flask. The filter paper and residue were removed and placed back in the reflux beaker. Concentrated $\mathrm{HCl}(5 \mathrm{~mL})$ was added and heated at $95 \pm 5^{\circ} \mathrm{C}$ until the filter paper dissolved. The residue was filtered and the filtrate was collected in the same flask. The cover and sides of the reflux beaker were washed with $\mathrm{HCl}$ and this solution was also added to the flask. A control sample was prepared by following the entire sample preparation procedure without sludge. The sample was diluted 1:1 using concentrated ammonium hydroxide followed by a 1000-fold dilution with background electrolyte before injecting on the microchip. The lead concentration was determined based on the actual weight of the dried sludge sample and the final dilution vol- 
umes. The certified metal contents in this material by inductively coupled plasma - mass spectrometry (ICP-MS) are shown in Table 1.

Table 1. Certified metal content in electroplating sludge reference material.

\begin{tabular}{|c|c|c|c|c|c|}
\hline Element & Concn. ${ }^{*} \mathrm{Mg} / \mathrm{Kg}$ & Element & Concn. $\mathrm{Mg} / \mathrm{Kg}$ & Element & Concn, $\mathrm{Mg} / \mathrm{Kg}$ \\
\hline $\mathrm{Al}$ & $692.5 \pm 82.5$ & $\mathrm{Ba}$ & $173.3 \pm 23.5$ & $\mathrm{Ca}$ & $562.7 \pm 33.0$ \\
\hline $\mathrm{Cr}$ & $79.5 \pm 14.1$ & $\mathrm{Cu}$ & $63,169.3 \pm 2410.0$ & $\mathrm{Fe}$ & $2,698.7 \pm 814.5$ \\
\hline $\mathrm{Pb}$ & $119,344.0 \pm 27453.0$ & Mg & $(80.0)$ & $\mathrm{Mn}$ & $17.5 \pm 2.1$ \\
\hline $\mathrm{Hg}$ & (1.4) & $\mathrm{Ni}$ & $193.6 \pm 15.0$ & $\mathrm{Ag}$ & $56.4 \pm 6.3$ \\
\hline $\mathrm{Na}$ & $(1,576.2)$ & $\mathrm{Zn}$ & $182.6 \pm 41.0$ & & \\
\hline \multicolumn{6}{|c|}{$\begin{array}{l}\text { * Certified and noncertified values from vendor, values in parentheses are not certified; certified values are determined c } \\
\text { a dry weight basis; uncertainties are one standard deviation of the measurement; the uncertainty is obtained from 95\% } \\
\text { confidence intervals. }\end{array}$} \\
\hline
\end{tabular}

For this assay, the standard addition method was used to account for matrix effects. In this electroplating sludge sample, it was also observed that copper (at a two-fold higher molar concentration) partially quenched the fluorescence of cleaved DNA, resulting in a systematic error in the quantitative detection of $\mathrm{Pb}^{2+}$. Since the solubility constant of $\mathrm{Pb}(\mathrm{OH})_{2}(\mathrm{Ksp}=$ $\left.2.5 \times 10^{-16}\right)$ is three orders of magnitude larger than that of $\mathrm{Cu}(\mathrm{OH})_{2}(\mathrm{Ksp}=$ $\left.1.6 \times 10^{-19}\right)$, it was possible to effect quantitative removal of copper in the sludge digestate as a copper hydroxide precipitate at the electrolyte $\mathrm{pH}$ of $\geq 8$. We confirmed that copper ion was removed to an undetectable level using the laboratory $\mathrm{CE}$ instrument. The lead ion, on the other hand, gave a quantitative recovery at $\mathrm{pH} 8$ and the enzymatic DNA reaction was even more efficient than at $\mathrm{pH} 7$, showing faster reaction times in the microfluidic device. Other metal ions did not interfere in the quantitative determination of lead.

Figure 8 shows the standard addition curve for the determination of lead in the sludge sample. The sludge digestate was prepared as described earlier but four aliquots were spiked with a $50 \mathrm{mM} \mathrm{Pb}^{2+}$ standard solution to make final added concentrations of 7, 12, 52, and $102 \mu \mathrm{M} \mathrm{Pb2}{ }^{+}$producing a five-point calibration including the nonspiked sludge sample. The experiments were conducted in the same manner as the calibration using the 
lead standard in buffer solution. The calibration plot had a correlation coefficient of 0.9993. The concentration of lead in the standard electroplating sludge reference material was determined to be $\left[\mathrm{Pb}^{2+}\right]=125,200 \pm$ $3,756 \mathrm{mg} / \mathrm{kg}$, a value within $4.9 \%$ of the certified value of $119,344 \mathrm{mg} / \mathrm{kg}$, indicating the potential for excellent accuracy of this microfluidic / DNAzyme system for $\mathrm{Pb}^{2+}$ determination.

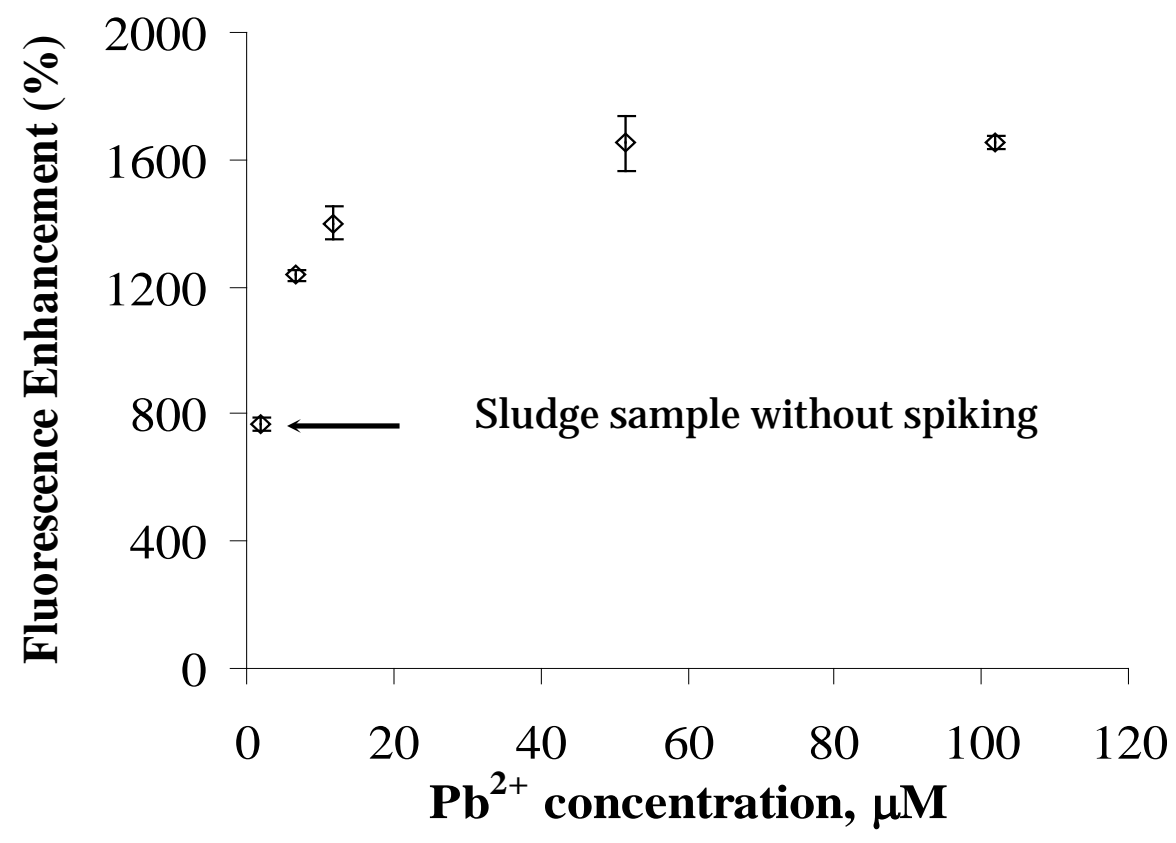

Figure 8. Plot of fluorescence enhancement as a function of lead ion concentration spiked in the electroplating sludge sample for standard addition calibration. Error bars represent $\pm 1 \sigma(n=4)$.

This work represented the initial steps toward creation of a robust field sensor for lead in ground water or drinking water. This research is detailed in Chang et al. 2005. 


\section{Materials and Methods}

Over the course of this project, the numerous tasks required copious supplies. While we made every effort to report all materials and equipment used, it should be noted that the critical items for this work were the DNA sequences, the molecular gate membranes, and the polymer assembly materials. The methods for each part of the project will be described in the next section, where the results are discussed.

\subsection{General chemicals and materials}

Lactic acid and ammonium hydroxide were obtained from Fisher Scientific (Fair Lawn, NJ ). HEPES (N-[2-hydroxyethyl]piperazine-N'-[2ethanesulfonic acid]), sodium chloride and sodium hydroxide were purchased from Aldrich (Milwaukee, WI). Prepolymer and curing agent (Sylgard 184, Dow Corning Corp. Midland, MI) and polycarbonate nuclear track-etched (PCTE) membranes with a hydrophilic wetting layer of poly(vinylpyrrolidine) (Osmonics, Minnetonka, MN) were used in the PDMS (poly(dimethylsiloxane)) chip. These PCTE membranes are $10 \mu \mathrm{m}$ thick with $200 \mathrm{~nm}$ diameter pores at a pore density of $3 \times 10^{8}$ pores $/ \mathrm{cm}^{2}$. The lead stock solutions ( $1000 \mathrm{mg} / \mathrm{L}$ ) were purchased from Fisher Scientific as an atomic absorption standard solution in $2 \% \mathrm{HNO}_{3}$. Working solutions of lower concentration were prepared by serial dilution of the stock solution with a background electrolyte (BGE). The BGE (25 mM lactate, 25 $\mathrm{mM}$ HEPES, and $50 \mathrm{mM} \mathrm{NaCl}$ ) was prepared by dissolving lactic acid, HEPES and NaCl in deionized water (18.2 M , Milli-Q UV-plus system, Millipore, Bedford, MA). The $\mathrm{pH}$ of the electrolyte was adjusted to 7 with ammonium hydroxide. Calibration of the chip sensor was performed using seven different lead concentrations. All reagents were analytical grade or higher.

\subsection{Preparation of DNAzyme}

The fluorescently labeled oligonucleotides were purchased from Integrated DNA Technology Inc. (IDT of Coralville, IA). The design of lead DNAzymes is described in detail by Liu and Lu (2003). Briefly, 3' end Dabcyl (4-(4'-dimethylaminophenylazo)benzoic acid)-labeled enzyme strand, termed 17E-Dy, and 5' end FAM (6-carboxyfluorescein)) and 3' end Dabcyl-labeled cleavable DNA substrate, termed 17DS-FD, were chosen 
(Figure 9). The DNAzyme enzyme-substrate complex was prepared with $500 \mathrm{nM}$ of both 17E-Dy and 17DS-FD for LIF measurements and $2.5 \mu \mathrm{M}$ of both enzyme and substrate for fluorescence microscopy studies. A sample of enzyme and substrate was heated at $90{ }^{\circ} \mathrm{C}$ for $2 \mathrm{~min}$ and slowly cooled to $5^{\circ} \mathrm{C}$ for $2 \mathrm{hr}$ to anneal the strands together and create the complex. Note that the FAM is quenched both intermolecularly by the enzyme Dabcyl and intramolecularly by the substrate Dabcyl.

Fluorescence quenched

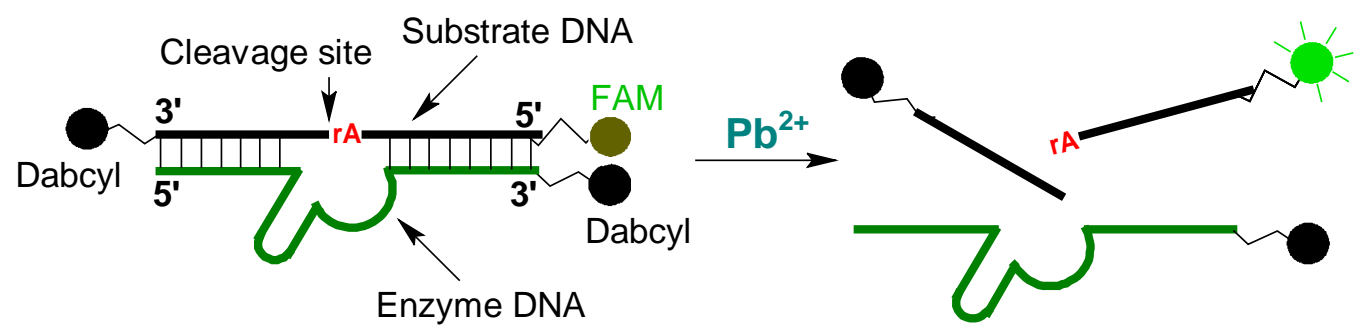

Figure 9. The sensor is composed of a dual-labeled cleavable substrate DNA whose 5'- and 3'end is labeled with a fluorophore (FAM) and a quencher (Dabcyl), respectively, and an enzyme strand whose 3'-end is labeled with a Dabcyl. Initially, the fluorescence of FAM is quenched because of the close proximity of the Dabcyl. In the presence of $\mathrm{Pb}^{2+}$, the substrate DNA is cleaved, resulting in the release of fragments and a concomitant increase in fluorescence.

The required DNA sequences and the sensing mechanism for the leadspecific construct are shown in Figure 1 (Section 1.1). In the pictured version, the substrate DNA is fluorescently labeled at the $5^{\prime}$ end, while the enzyme strand possesses a quencher at the 3 ' end. The labeling is arbitrary, however, and depending on the desired behavior, these modifications can be reversed so that the enzyme strand carries the fluorescent tag. The DNA enzyme strand, 17Ea, and the DNA/ RNA chimeric substrate strand, 17Sa, with modifications were purchased from IDT and used after HPLC purification. The oligonucleotide sequences are shown in Table 2, where the DNA labels have been selected to describe the modifications. Extra bases (TTTTTAAACGTA) have been added to the 5' end of the enzyme strand to extend the active region away from a surface. Streptavidin and FITC labeled streptavidin were purchased from Sigma-Aldrich (St. Louis, MO) and used without additional purification. Planar PMMA substrates $(1 \mathrm{~mm}$ thickness) were purchased from Goodfellow (Oakdale, PA) for immobilization experiments outside the microfluidic device. 
Table 2. DNA labels and sequences.

\begin{tabular}{|l|l|}
\hline DNA labels & Sequence \\
\hline Bio-5T-17Ea(7)-FI & 5'-/5Bio/TTT TTA AAC GTA CAT CTC TTC TCC GAG CCG GTC GAA ATA GTG AGT/36-FAM/-3' \\
\hline Fl-anti17E(7) & 5'-/56FAM/ACT CAC TAT TTC GAC CGG CTC GGA GAA GAG ATG TCT CTT T-3' \\
\hline $\begin{array}{l}\text { Bio-5T-17Ea(7)- } \\
\text { Alexa }\end{array}$ & 5'-/5Bio/TTT TTA AAC GTA CAT CTC TTC TCC GAG CCG GTC GAA ATA GTG AGT/36-Alexa/-3' \\
\hline (7)17Sa-IB & 5'-/5IAbFQ/ACT CAC TAT rAGG AAG AGA TGT ACG TTT-3' \\
\hline
\end{tabular}

\subsection{Microfluidic device}

\subsubsection{PDMS devices}

Details of the channel layout and fabrication of multilevel microfluidicnanofluidic hybrid architectures have been provided previously (Kuo et al. 2003). A three-dimensional transport device was depicted schematically in Figure 4. Two identical channels (50 $\mu \mathrm{m}$ wide, $30 \mu \mathrm{m}$ deep, and $14 \mathrm{~mm}$ long) were orthogonally oriented on a PDMS microchip and separated at the intersection by a nanocapillary array interconnect (NAI) made from track-etched polycarbonate (PCTE). The PCTE membrane possesses 200 nm diameter cylindrical pores. Platinum wires ( $250 \mu$ m diameter, Goodfellow Corp., Berwyn, PA), mounted into reservoirs at the distal ends of the microchannels, were used to apply bias voltages. An 8-relay system, designed to switch electrical contacts between Pt electrodes and high voltage power supplies (Bertan High Voltage, Hicksville, NY) for different configurations and magnitudes of microfluidic manipulation, was computercontrolled via a multifunction data acquisition card (DAQ) (National Instruments Corp., Austin, TX) and Labview software (National Instruments Corp.).

\subsubsection{PMMA devices}

A microfluidic/ nanofluidic hybrid device with nanopore diameter of 200 nm was fabricated using the approach described previously (Flachsbart et al. 2006). Figure 10 shows the microfluidic device consists of three PMMA layers (each $20 \mu \mathrm{m}$ thick), one nuclear track-etched polycarbonate (PCTE) NCAM (6-10 $\mu \mathrm{m}$ thick; GE Osmonics, Minnetonka, MN) sandwiched between two microfluidic-channel-containing PMMA layers, and a polycarbonate cap layer ( $1.5 \mathrm{~mm}$ thick) with drilled reservoirs ( $4 \mathrm{~mm}$ diameter). The separation channel with a length of $2.80 \mathrm{~cm}$ spatially bisects the 1.30 $\mathrm{cm}$ long detection channels. The rectangular channels are $100 \mu \mathrm{m}$ in width and $20 \mu \mathrm{m}$ in depth. Fluidic communication between separation and de- 
tection channels is achieved via the NCAM which projects a $100 \times 100 \mu \mathrm{m}^{2}$ active area at the cross-section of the microfluidic channels in the upper and lower layers.

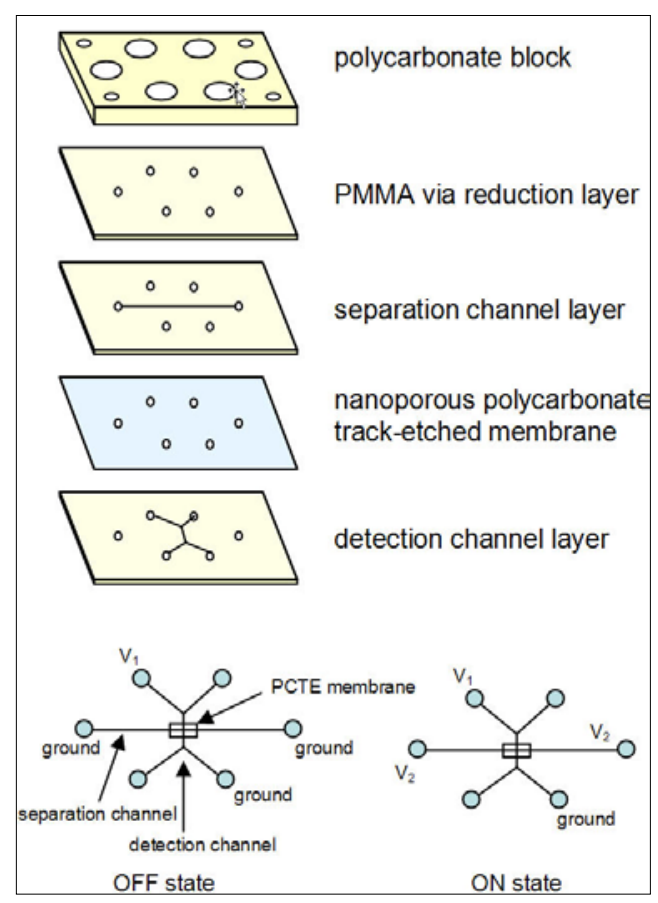

Figure 10. Schematic diagram showing the various layers in the PMMA microfluidicnanofluidic device and the potential program used to control fluid flow between the channels.

(Top) Exploded schematic diagram showing the constituent layers individually. (Bottom) Diagram showing terminals and voltage programs used to realize the OFF and ON states. The central rectangle represents the NCAM (PCTE membrane) that controls fluidic communication between microfluidic layers.

\subsubsection{DNAzyme Spot Device}

One simple method we investigated was to form an enclosed microfluidic chip using a PMMA substrate and a PDMS slab that contains the microchannel structure and sample ports as shown in Figure 11. 


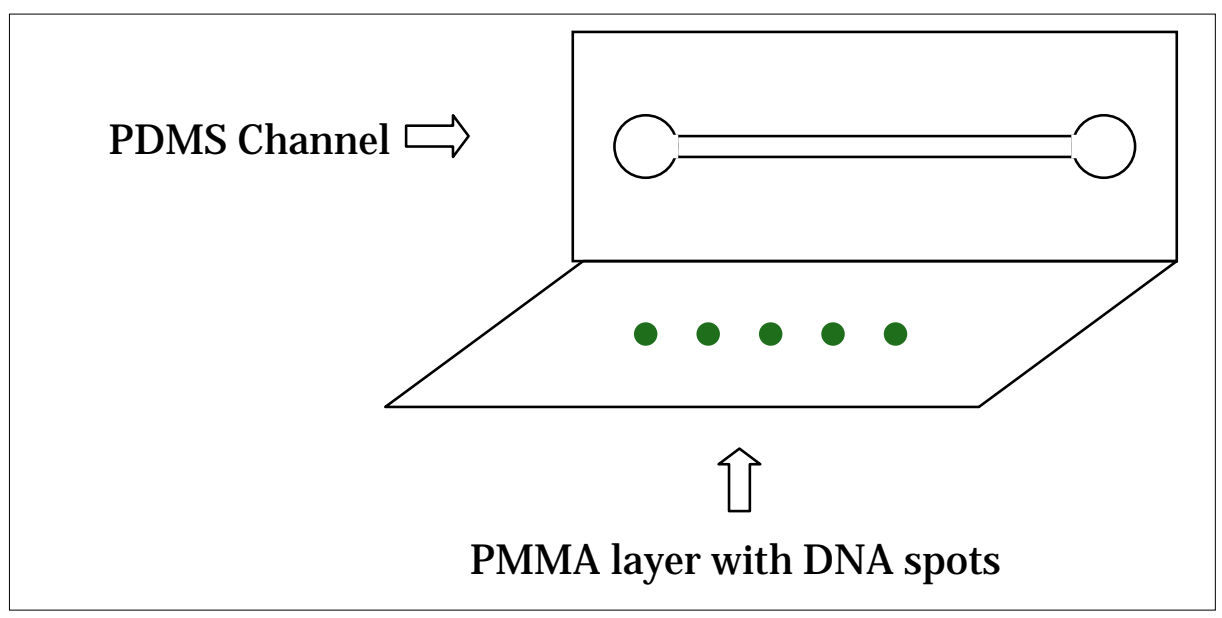

Figure 11. Design for fabrication of microfluidic chip for lead sensing.

Standard rapid prototyping and soft lithography processes were used to fabricate a PDMS layer with a single, embedded channel and distal reservoirs. A 10:1 mixture of poly (dimethylsiloxane) prepolymer and curing agent (Sylgard 184, Dow Corning) was used and cured at room temperature for at least $24 \mathrm{~h}$ to form a PDMS layer. The sealing between the PDMS and PMMA layers was achieved by oxygen plasma treatment of the PDMS surface for $20 \mathrm{~s}$ at $55 \mathrm{~W}$ with $\mathrm{O}_{2}$ pressure of ca. 500 mTorr. The channel is $100 \mu \mathrm{m}$ wide, $20 \mu \mathrm{m}$ deep, and $20 \mathrm{~mm}$ long. A reversible sealing was achieved by putting the PDMS layer and the modified PMMA under vacuum for at least $24 \mathrm{~h}$. The channel bisects each DNAzyme spot so that the enzyme beneath the PDMS remains unhybridized and can act as an internal standard, while substrate DNA flowing within the channel can hybridize a $100 \mu \mathrm{m}$ wide stripe through each spot.

\subsection{DNAzyme immobilization}

A PMMA substrate was cleaned with isopropanol and water, and then dried in nitrogen. Biotinylated DNAzyme labeled with fluorescein, Bio-5T17Ea(7)-Fl and the control non-biotinylated DNA labeled with fluorescein, Fl-anti17E(7), were dissolved in 1X MicroSpotting solution Plus (Telechem International Inc., Sunnyvale, CA) to make $20 \mu$ M solutions. Streptavidin $(1 \mathrm{mg} / \mathrm{mL}$ ) was spotted on the surface and allowed to dry for 15- $20 \mathrm{~min}$, followed by spotting of the DNAzyme solution. The slide was left overnight in a chamber at $<30 \%$ relative humidity and then treated with superstreptavidin blocking buffer (20 $\mu \mathrm{L}$ ) (Telechem) under a coverslip for $1 \mathrm{~h}$, washed sequentially with Wash Buffers A and B for 5 min each, immersed in Wash Buffer C for 1s (Telechem), then dried with nitrogen. 
For immobilization of DNAzyme in a PMMA microfluidic device, streptavidin solution $(1 \mathrm{mg} / \mathrm{mL})$ was electrokinetically driven through the detection channel, left overnight, rinsed, and then followed by flowing biotinylated DNAzyme labeled with Alexa 488, Bio-5T-17Ea(7)-Alexa, dissolved in 1X MicroSpotting Solution Plus. The device was left overnight in a chamber at $<30 \%$ relative humidity, then the channel was treated with superstreptavidin blocking buffer, washed with Wash Buffers A, B and C and then filled with $50 \mathrm{mM}$ tris acetate buffer (pH 7.2) +50 mM NaCl.

We also had success immobilizing biotinylated DNAzyme onto superstreptavidin glass (SSG; Telechem) by following similar spotting instructions as given above. In this way, we could use either PMMA or glass as one of the constituent device layers.

\subsection{Hybridization and detection of lead}

The quencher-labeled substrate DNA, (7)17Sa-IB, was dissolved in hybridization buffer $(50 \mathrm{mM}$ tris acetate $(\mathrm{pH} 7.2)+1 \mathrm{M} \mathrm{NaCl})$ to make a $5 \mu \mathrm{M}$ solution. Hybridization on the planar PMMA substrate (Figure 11) was carried out by placing quencher-labeled substrate DNA onto the DNAzyme spots, followed by addition of excess hybridization buffer under a coverslip, and leaving overnight in a humidified chamber in order to prevent drying. The slide was then kept at $4{ }^{\circ} \mathrm{C}$ for $30 \mathrm{~min}$ and warmed to room temperature for $15 \mathrm{~min}$, followed by washing and drying.

Prior to the lead reaction, the PMMA substrate was soaked in $50 \mathrm{mM}$ tris acetate buffer ( $\mathrm{pH} 7.2)+50 \mathrm{mM} \mathrm{NaCl}$ for $5 \mathrm{~min}$ in order to remove physically adsorbed or unhybridized substrate DNA. After a reaction with $\mathrm{Pb}^{2+}$, the substrate was washed again with $50 \mathrm{mM}$ tris acetate buffer $(\mathrm{pH} 7.2)+$ $50 \mathrm{mM} \mathrm{NaCl}$ for $5 \mathrm{~min}$, rinsed with water, and dried.

Hybridization in a microchannel (Figure 10) was carried out by filling the DNAzyme immobilized PMMA channel with quencher-labeled substrate DNA and leaving overnight at $25^{\circ} \mathrm{C}$ in a humidified chamber. The device was then kept at $4{ }^{\circ} \mathrm{C}$ for 30 min and warmed to room temperature for 15 min, followed by washing with Wash Buffers $\mathrm{A}, \mathrm{B}$ and $\mathrm{C}$. $\mathrm{Pb}^{2+}$ was introduced into this prepared detection channel via electrokinetic flow. After the reaction with $\mathrm{Pb}^{2+}$, the channel was washed again with $50 \mathrm{mM}$ tris acetate buffer $(\mathrm{pH} 7.2)+50 \mathrm{mM} \mathrm{NaCl}$ for $5 \mathrm{~min}$. In all cases, the channel was filled with $50 \mathrm{mM}$ tris acetate buffer $(\mathrm{pH} 7.2)+50 \mathrm{mM} \mathrm{NaCl}$ before fluorescence imaging. 


\subsection{Electrokinetic flow}

$\mathrm{Pb}^{2+}$ containing solution was electrokinetically injected into the detection channel (or receiving channel) from the separation channel (or source channel) through the NCAM. All solutions were filtered through $0.45-\mu \mathrm{m}$ filters obtained from Nalgene (Rochester, NY, USA). The separation channel was filled with $10 \mu \mathrm{M}$ lead acetate in $25 \mathrm{mM}$ tris acetate (pH 7.2) + 25 $\mathrm{mM}$ lactic acid $+50 \mathrm{mM} \mathrm{NaCl}$ and the detection channel containing immobilized DNAzyme was filled with the buffer alone $(25 \mathrm{mM}$ tris acetate buffer ( $\mathrm{pH} 7.2)+25 \mathrm{mM}$ lactic acid $+50 \mathrm{mM} \mathrm{NaCl}$ ). This buffer composition was found to be optimum for electrophoresis as well as DNAzyme activity (Chang et al. 2005). The channels were filled by adding the respective solution to one of the reservoirs and pressure filling the channel using a syringe. Platinum (Pt) wires were used as electrodes to establish contact with the buffer in the reservoirs. A linear current-voltage relationship in each of the channels indicated electrical continuity and the absence of air bubbles in the channels. The device was also observed under a Leica fluorescence microscope (Model DMIRE2, Leica Microsystems Wetzlar $\mathrm{GmbH}$, Germany) to ensure complete filling of the channels and absence of air bubbles. Potentials were applied using a power supply, and potential control and EOF current measurements were achieved using Labview (National Instruments) software. The potentials used in each experiment are shown in later sections. After each experiment, the system was switched to the "off" state and left for 5 min. During this time, the injection of $\mathrm{Pb}^{2+}$ through the membrane stops, and residual $\mathrm{Pb}^{2+}$ in the detection channel was flushed out by the flowing buffer.

\subsection{Regeneration}

Regeneration of the sensing construct in channels or on spots followed the identical procedures given above for hybridization. Specifically, on spots, 5 $\mu \mathrm{M}$ solutions of quencher labeled substrate DNA in $50 \mathrm{mM}$ tris acetate plus $1 \mathrm{M} \mathrm{NaCl}$ were placed on the enzyme DNA spots overnight in a humidified chamber, then kept at $4{ }^{\circ} \mathrm{C}$ for $30 \mathrm{~min}$, then room temperature for $15 \mathrm{~min}$, washed, and dried. In channels, the detection channel was filled with $5 \mu \mathrm{M}$ substrate DNA in hybridization buffer and left overnight at $25^{\circ} \mathrm{C}$ in a humidified chamber. The device was then kept at $4{ }^{\circ} \mathrm{C}$ for 30 min and subsequently warmed to room temperature for $15 \mathrm{~min}$, followed by washing with Wash Buffers A, B, and C. 


\subsection{Fluorescence microscopy}

Fluorescence microscopy was used for signal acquisition by using an inverted Olympus epi-illumination microscope (Melville, NY). The CCD camera (J avelin Ultrichip Hi Res, Torrance, CA) output was recorded with a videocassette recorder and a computer-controlled video capture device (ATI Technologies, Markham, Ontario, Canada). Fluorescence was excited with $488 \mathrm{~nm}$ radiation from an $\mathrm{Ar}^{+}$ion laser (Innova 300, Coherent Inc., Santa Clara, CA) which is very close to the FAM $492 \mathrm{~nm}$ excitation maximum. The laser light was passed through a set of irises and a neutral density filter (Newport, Irvine, CA) before reaching a dichroic mirror (505DCLP, Chroma Technology Corp., Brattleboro, VT). The excitation light was focused by a $10 \mathrm{X}$ objective for a $50 \mu \mathrm{m}$ diameter area of interrogation. Fluorescence signals were collected by the same lens and dichroic mirror assembly and optically filtered through a $100 \mu \mathrm{m}$ pinhole and bandpass filter (HQ525/50m, Chroma Technology Corp.) that permits passage of the $518 \mathrm{~nm}$ FAM emission maximum before being detected by a photomultiplier tube (PMT) (HC124, Hamamatsu Corp., Bridgewater, NJ). Control of the PMT data collection was achieved through computer with a Labview program and data acquisition card (DAQ, National Instruments Corp.). All fluorescence signals were collected at the intersection of the crossed channels, (i.e., just below the nanocapillary array interconnect membrane).

Fluorescence images were acquired by using a fluorescence microscope (Zeiss Axiovert 200M, Thornwood, NY), equipped with a Hg lamp band filter (X-Cite, Mississauga, Ontario). The images were obtained using a 10X objective and recorded on a desktop computer with Axiovision software. The fluorescence signal intensity relative to the background was quantified using pixel counts obtained directly from specific regions in the images. The intensity values were obtained by taking the average pixel count from two different regions of the channel on either side of the point of intersection. 


\section{Results and Discussion}

\subsection{Microfluidic device design}

The field of microfluidics is often viewed as the next-generation technology for rapid DNA sequencing, high-throughput drug screening, and ultrasensitive chemical analysis. There is a need to develop microfluidic and nanofluidic devices and chips consisting of an integrated optical sensor which can measure and record, directly and without external intervention, the unique dielectric response of material flowing through the device. Additionally, a need exists for an integrated chip that is capable of easily identifying analytes in different fluids, including aerosols, solvents and buffers of varying ionic concentration. In a particular configuration, the devices detect or measure changes in conductance of a fluid, molecule, particle or cell as it passes through the device. This section describes the work done to create the injection and detection system for chip devices.

\subsection{Design overview}

As shown in Figure 10 in Section 3, the fabrication of the PMMA-based microfluidic system uses a multi-layered design. A polycarbonate nuclear track-etched (PCTE) membrane of pore size $200 \mathrm{~nm}$ is sandwiched between the PMMA microfluidic layers and acts as a fluidic gate via its array of nanometer diameter pores (the nanocapillary array). The fluidic gate can be "opened" or "closed" by applying appropriate bias voltages to control the transport of analytes between crossed microfluidic channels. Analytes injected into a separation channel thus can be "captured" by opening the gate and transferring the analytes into a second, vertically displaced, microfluidic channel. Since the PMMA surface is negatively charged, the direction of EOF of the buffer is from the positive potential to ground (i.e., anode to cathode) in all the microchannels, which is also the electromigration direction of $\mathrm{Pb}^{2+}$. When the separation channel is grounded ("off" state), there is no injection of $\mathrm{Pb}^{2+}$ across the NCAM 22 . When a positive potential is applied to the separation channel as shown in "on" state diagram (Figure 10), the positively charged $\mathrm{Pb}^{2+}$ ions move from the separation channel across the membrane to the detection channel towards the ground and, in the process, react with the immobilized DNAzyme in this channel. To inject $\mathrm{Pb}^{2+}$, large positive voltages $\left(\mathrm{V}_{2}\right)$ were applied to the two reservoirs of the separation channel and one of the res- 
ervoirs of the cross channel $\left(\mathrm{V}_{1}\right)$, while the other reservoir of the cross channel was grounded. The bias program used (i.e., $V_{2}>V_{1} / 2$ ), ensures rapid injection of $\mathrm{Pb}^{2+}$ between the microchannels because the electrophoretic transport of $\mathrm{Pb}^{2+}$ is in the same direction as $\mathrm{EOF}$, and the apparent transport velocity is equal to the vector sum of EOF and electrophoresis.

\subsubsection{Molecular gate and electroosmotic flow}

The molecular gate device structure was replicated in a PMMA construct (Flachsbart et al. 2006). Figure 12 shows three representative devices with different NCAMs (nanocapillary array membranes) incorporated within the PMMA hybrid nano-microfluidic construct, using the assembly process shown in Figure 10. The separation channel in the middle of the device is currently $28 \mathrm{~mm}$ long, but could be lengthened if needed via serpentine pattern.

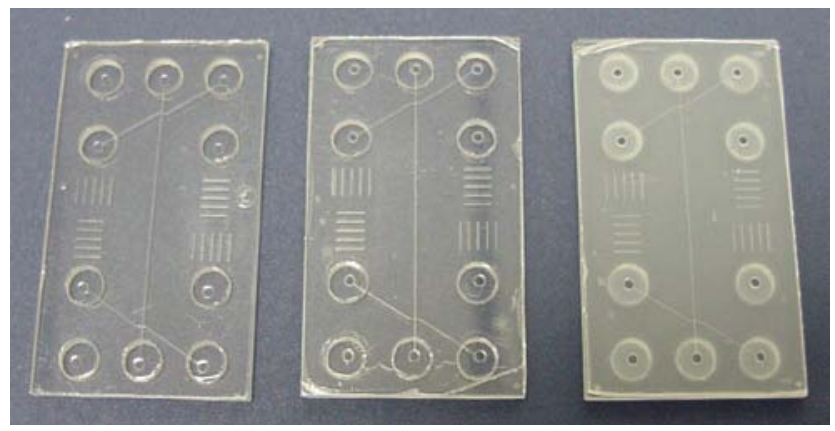

Figure 12. Three molecular gate devices with 10,50, and $220 \mathrm{~nm}$ diameter pore NCAMs (from left to right, respectively). The NCAM extends across the entire device and is positioned between the cross channels and over the separation channel.

Electrical characterization consisted of measuring microfluidic channel resistivity and determining the electroosmotic flow (EOF) coefficients along the channels at different $\mathrm{pH}$ values. Linear I-V plots $\left(\mathrm{R}^{2}>0.995\right)$ using $10 \mathrm{mM}$ phosphate buffer (PB) ( $\mathrm{pH}$ 7.4) were obtained yielding a mean of $26.7 \pm 0.4 \mathrm{M} \Omega / \mathrm{cm}$ along the longer microfluidic channel $(\Delta \mathrm{l}=$ $2.80 \mathrm{~cm})$ and $37.6 \pm 0.4 \mathrm{M} \Omega / \mathrm{cm}$ along the shorter cross-channels $(\Delta \mathrm{l}=$ $1.23 \mathrm{~cm}$ ). Measurements were stable and reproducible for 7 days. No measurable leakage current was observed through the chip itself, indicating no discernable fluid leaks between levels. The EOF mobilities versus solution $\mathrm{pH}$, given in Table 3 , were measured with the current monitoring method using $5 \mathrm{mM}$ and $10 \mathrm{mM}$ phosphate buffer (PB) solutions at $100 \mathrm{~V}$. These EOF values are within a factor of two of published EOF values for PMMA microfluidic channels, which are noted to vary with processing method (Chen et al. 2006). Transport through the nanofluidic intercon- 
nects (across the NCAM membrane) was demonstrated by filling a cross channel with $1 \mu \mathrm{M}$ of green fluorescent protein (GFP) and electrokinetically transporting analyte plugs (with $600 \mathrm{~V}$ pulses, 2 seconds in duration) into the middle separation channel. A 488-nm Ar+ laser was focused on the receiving channel away from the intersection to produce the laser induced fluorescence (LIF) graph shown in Figure 13. Plug injections were confined and reproducible.

Table 3. Electroosmotic coefficients $\left\{\mathrm{cm}^{2} / \mathrm{V} \cdot \mathrm{s}\right\}$ for phosphate buffer solution versus $\mathrm{pH}$ measured using the current monitoring method.

\begin{tabular}{|l|l|l|}
\hline pH & Cross Channel Ave. & Separation Channel \\
\hline 8.8 & $2.7 \pm 0.6 \times 10^{-4}$ & $2.2 \pm 0.3 \times 10^{-4}$ \\
\hline 7.3 & $2.8 \pm 0.5 \times 10^{-4}$ & $3.5 \pm 0.7 \times 10^{-4}$ \\
\hline 4.4 & $2.5 \pm 0.9 \times 10^{-4}$ & $2.2 \pm 0.3 \times 10^{-4}$ \\
\hline
\end{tabular}

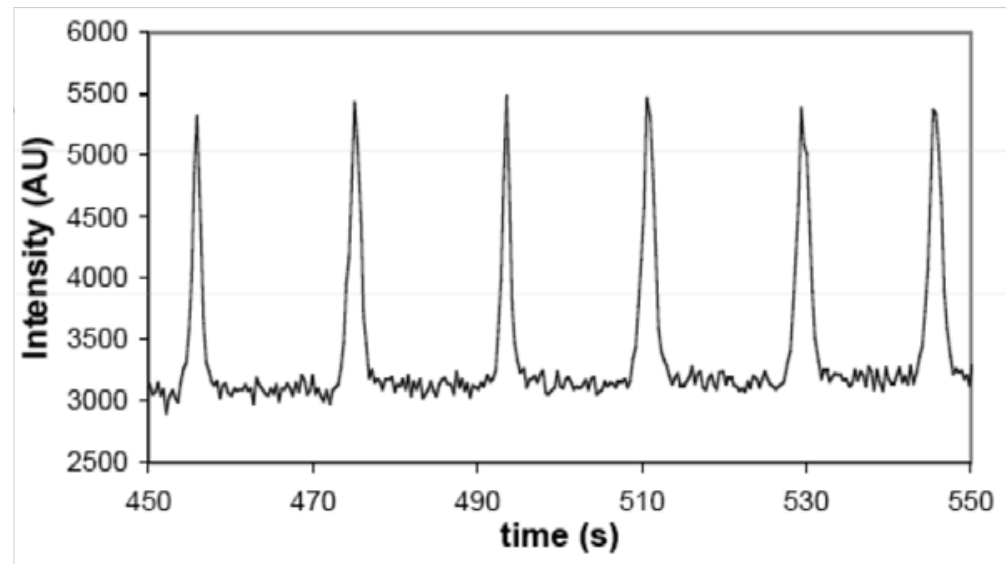

Figure 13. Laser-induced fluorescence (LIF) detection of $1 \mu \mathrm{M}$ GFP in $10 \mathrm{mM}$ phosphate buffer injected for $2 \mathrm{~s}$ at $600 \mathrm{~V}$ from one microfluidic channel, across a NCAM, and into a second microfluidic channel containing the buffer solution only.

Double-membrane devices were fabricated as shown in Figure 14. These devices consisted of three microfluidic channels separated in the vertical direction (Z axis) by two NCAM layers. 


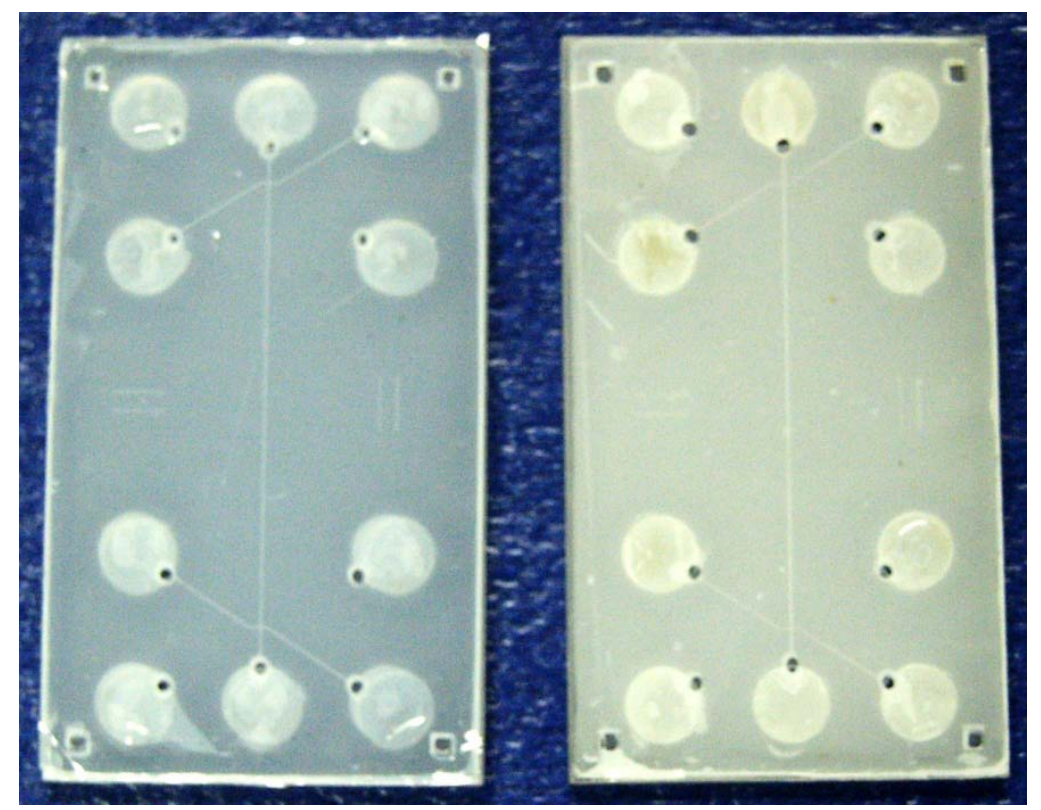

Figure 14. Double membrane samples with three microfluidic layers separated in the vertical direction by two NCAM layers. The device on the left has two $50 \mathrm{~nm}$ NCAMs and the device on the right has two $100 \mathrm{~nm}$ NCAMs. The separation channel is located between the NCAM layers with an injection and a collection channel at both ends.

A product of this research is the new device design shown in Figure 14. It enables conductivity detection of an electrophoretically separated sample of cationic metal species. The approach would be to electrokinetically inject the analyte from the cross channel labeled 4-7 in Figure 15, into the separation channel labeled 1-6. Application of an alternating current (AC) signal between the cross channels 3-10 and 2-9 could be monitored with a lock-in amplifier to detect slight changes in the resistivity of the separation channel region that they overlap (region shown in Figure 15 inset). As the injected analyte is electrophoretically separated and each metallic band passes between the intersecting cross channels at the other end, the bands can be detected by changes in resistivity between the cross channels. At the appropriate time interval for the lead band to arrive at this intersection, the voltage bias could be switched to pull this band into either cross channel, through the respective NCAM. Although earlier work demonstrated overlap between electrophoretically separated copper and lead bands in PDMS based devices, PMMA channels have a more stable surface chemistry and more reproducible EOF and are unlikely to have this problem. PMMA will be more similar to glass, where there is little difficulty in separating $\mathrm{Pb}$ from $\mathrm{Cu}$ as shown in Figure 16. This device is a novel approach to detecting metal species in solution by using conductivity without using a DNAzyme. 


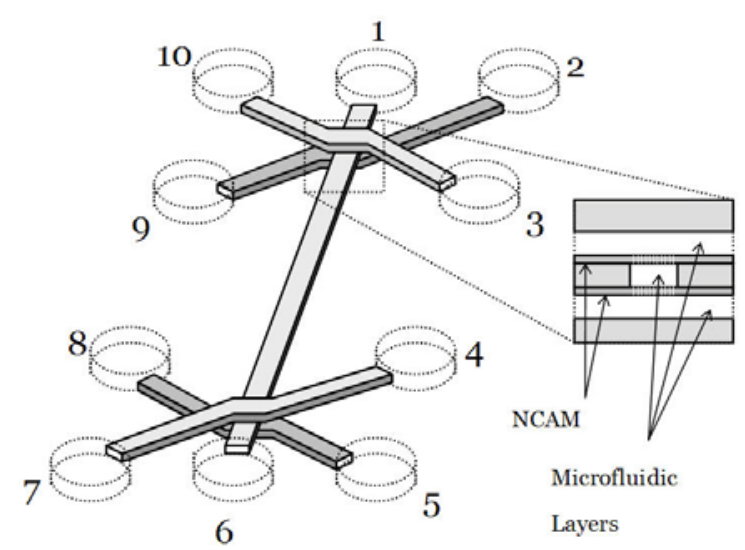

Figure 15. Relief schematic of a device showing the routing scheme of the microfluidic channels relative to each other. On the right is a cross-section of the intersection region where nanofluidic interconnects are formed between the microfluidic layers.

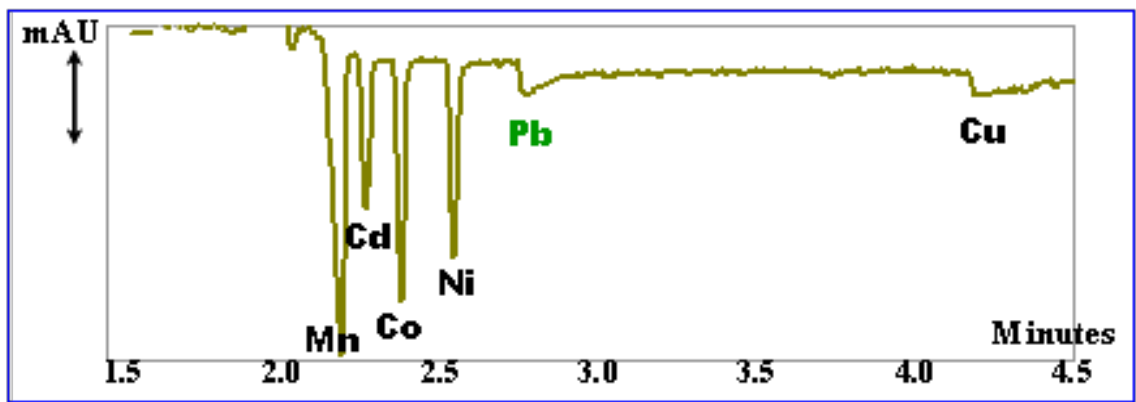

Figure 16. Glass capillary electrophoresis data demonstrating that distinct separation between the lead and copper bands is possible.

\subsubsection{Design problems}

Preliminary testing indicated that some design and fabrication changes needed to be made to the original design (Figure 14 and Figure 15). A further product of this research is the enhanced fabrication procedures defined in the following sections.

\subsubsection{Capacitor layer alignment}

The first design problem addressed was the one that showed measured capacitance was too low. The area of overlap was increased and methods to improve the alignment of the capacitive layers were worked on. The layer which contained the upper capacitor metal leads needed to be "flipped" before bonding to the device stack but this caused problems with metal layer cracking, wrinkling, and alignment. A new approach was developed to correct these problems. Individual layers were processed on carrier substrates, typically coverglass, and are released from these carriers when 
bonded to the device stack. This process was reproducible for the release of thick PMMA layers.

The new approach relied on the upper metal lead capacitor layer to be released by use of a thin (i.e., $1 \mu \mathrm{m}$ thickness) PMMA layer, which surprisingly occurred only partially or incompletely. These release conditions were drastically different than with the thicker layers. Attempts were made to use alternative substrates in place of coverglass with track etched polycarbonate closer to that of PMMA in order to facilitate the release of thin PMMA layers. Lastly, adhesion of the capacitor metal (Ni) to the PMMA layers has been significantly improved by applying a coating of Nickelex after Ni layer deposition, eliminating the bubbling and peeling of layers. However, the adhesion of Nickelex to PMMA was poor, and efforts focused on improving this adhesion.

For the new version, the device was modified to include two parallel plate capacitors in the middle of the separation channel as shown in the schematic and cross-sectional view shown in Figure 17.

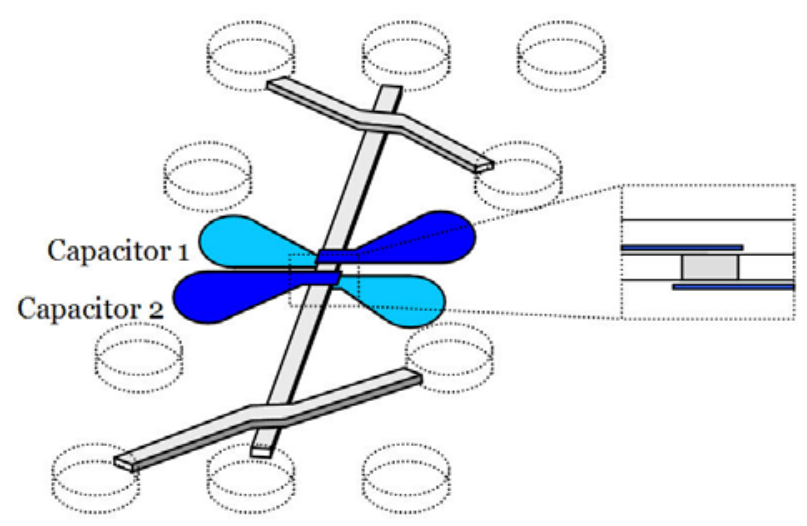

Figure 17. Schematic of the new microfluidic device design showing the two parallel plate capacitors located in the middle of the separation channel. The cross-sectional view shows the intended vertical overlap of one of the capacitive detectors.

The electronic interrogation method for this detector arrangement is shown in Figure 18 where a modified Wheatstone bridge was employed. Capacitors 1 and 2 refer to the two capacitors located on-chip. Resistors 1 and 2 were chosen to achieve a convenient resonant (or cutoff) frequency for measurements. The resonant (or cutoff) frequency for the equivalent 
low pass filter circuit was $f_{3 \mathrm{~dB}}=\frac{1}{2 \pi R C}$; at this frequency, small changes in the two capacitors will produce the greatest change in $\mathrm{V}_{\text {out }}$.

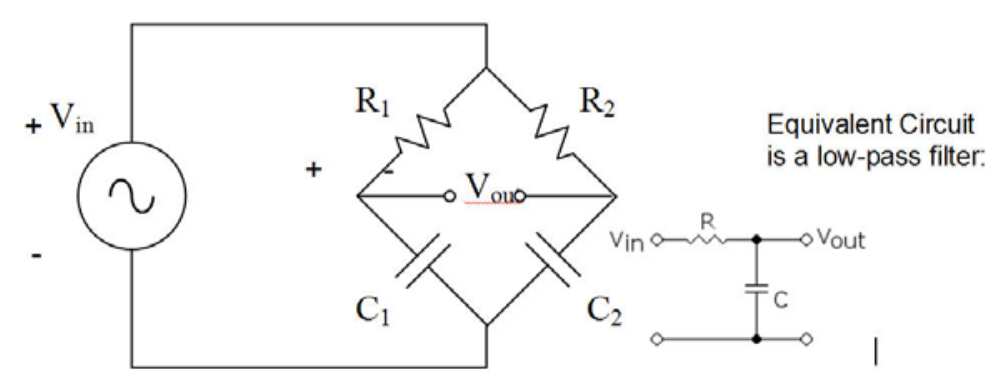

Figure 18. Left, electrical schematic used for detection, and on the right, its equivalent circuit, a low-pass filter. The modified Wheatstone bridge has the greatest $V_{\text {out }}$ sensitivity at the cutoff frequency of the equivalent circuit.

Following the realization of the design shown in Figure 17, two primary fabrication issues remained: (a) the layer-to-layer misalignment, caused by alignment shifting during bonding, and (b) the loss of integrity of the dielectric layer, necessary for sensitive capacitive interrogation of the channel region. Research eventually produced radically new fabrication processes and materials including a new adhesive and a new layer material.

\subsubsection{Layer-to-layer misalignment}

For optimal device capacitive detection, alignment between layers and the channel is critical. Previously, alignment often shifted during bonding of the layers. This alignment shifting was demonstrated to be related to coefficient of thermal expansion (CTE) mismatch between the temporary carrier of the layer and the device itself. This process is illustrated in Figure 19. To reduce and eliminate this problem, three research initiatives were pursued.

\subsection{1.1 Initiative 1: New adhesive developed}

To reduce shifting during bonding, a new adhesive was developed that would fix the position of the layers relative to each other at a lower temperature. This required the curing method to be modified. For thermally cured adhesives (such as previously used) to be stable at room temperature (i.e., have a long pot life) there needs to be a significant temperature difference between room temperature and the adhesive's cure tempera- 
ture. In general, the lower the cure temperature, the less stable the adhesive is at room temperature.

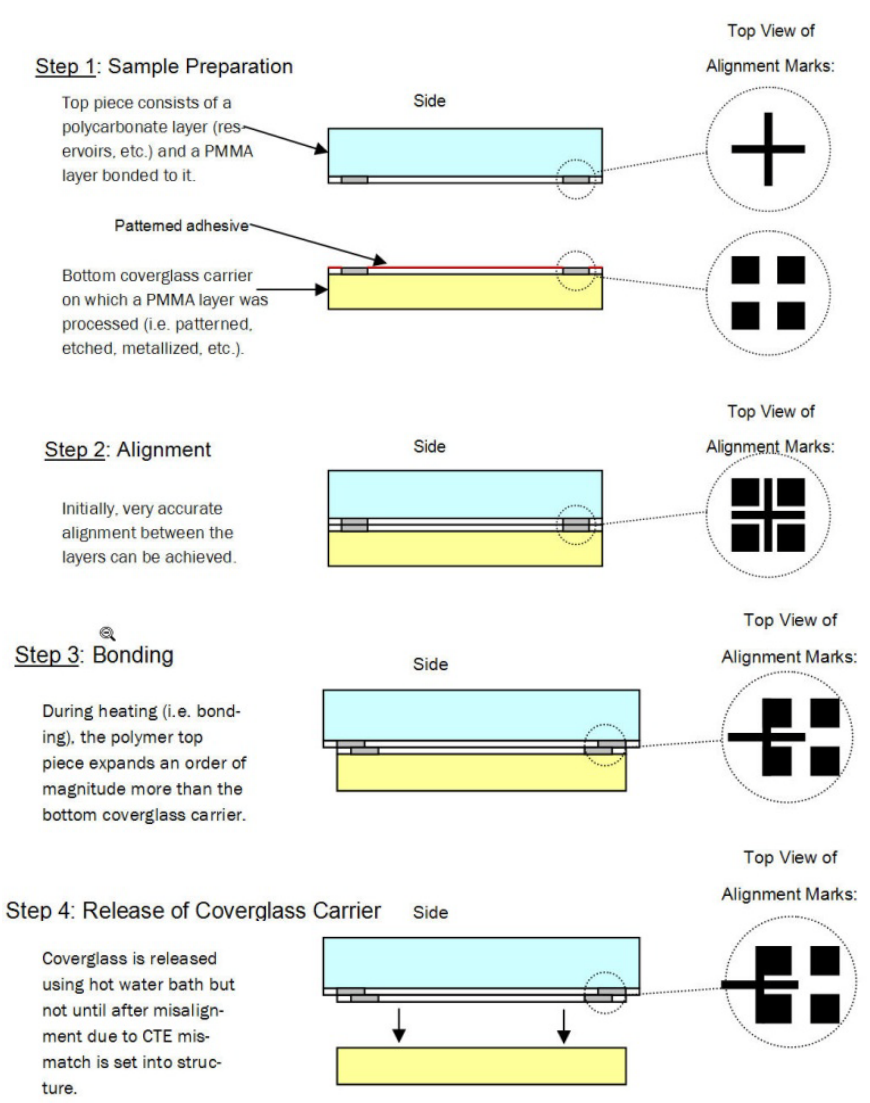

Figure 19. The four-step process to bond a second layer (already processed) to a device with a first layer. The second PMMA layer is affixed to a coverglass carrier for processing, and since the coverglass is much thicker than the PMMA (200 $\mu \mathrm{m}$ to $20 \mu \mathrm{m}$ ), it expands and contracts at the same CTE as the coverglass, which leads to layer misalignment.

Our previous adhesive was a thermally cured adhesive based on a Bisphenol-A epoxy, and its cure temperature could not be lowered significantly below $140{ }^{\circ} \mathrm{C}$. Ultraviolet (UV) curable adhesives, with and without the addition of a thermal curing component, were investigated. A new lower-temperature UV/ thermal curable adhesive was developed that lowered bond temperatures from $140{ }^{\circ} \mathrm{C}$ to fixing the layers relative to one another at $60{ }^{\circ} \mathrm{C}$. This translated to an almost $70 \%$ reduction in CTE stress during bonding while nearly eliminating the shifting during bonding.

This significantly lower bond temperature also was beneficial for the incorporation of the metal electrodes within the polymer device since CTE mismatches between the metal and the polymer also tended to result in stress fractures in the metal layer. Lowering the bond temperature helped 
reduce stress fractures in this metal layer and improve the overall integrity of the metal capacitive layer. The goal of reducing all the processing temperatures started with improving the critical bonding process.

It took three years to develop the previous adhesive, while the improved adhesive was developed in less than one year. This is a significant achievement considering that developing an adhesive for the fabrication of microfluidic devices requires that the adhesive be (a) UV and optically transparent, (b) contact printable with the ability to resolve $<100 \mu \mathrm{m}$ features, and (c) chemically and thermally stable. The new adhesive is based upon the SU8 epoxy group, and thus has improved chemical and thermal stability over the previous adhesive, as well as a surprising improvement in its ability to resolve features to $<60 \mu \mathrm{m}$.

\subsubsection{Initiative 2: Change in polymer layer}

The second initiative for reducing the detrimental effects of CTE was to change the polymer of the layers themselves to something that could be processed at a lower temperature. Previously, the polymer layers were made from PMMA, a material that requires a $180-200{ }^{\circ} \mathrm{C}$ temperature cure for reproducible thermal and chemical stability. This resulted in significant thermal stresses incorporated in the layers, due to the different CTE values of the polymer and the coverglass temporary substrate carrier on which it was produced.

It was decided to experiment with different polymers that could be cured at lower temperatures and the polymers that incorporated both a thermal and a UV cure mechanism showed the most promise for lowering processing temperatures. The new layer material was based on the SU8 epoxy group of polymers, but two common problems resulted from its use. First, SU8 shrinks during curing leaving cracks along channels. This can be minimized using stress relief openings spread throughout the layer or by the addition of flexibilizers to the formula. Because the incorporation of stress relief openings throughout the layer would create a serious design limitation on the various devices that could be made, we experimented with flexibilizers.

The second problem experienced with SU8 as a layer material is the nonuniformity of the coating. We experimented with 6 different flexibilizers as well as 12 different surfactants. The resulting formulation produced microfluidic channel layers with channel cracks reduced by a factor of $\sim 3$, and 
uniformity was improved by a factor of $\sim 4$. Figure 20 illustrates the two primary issues hindering the use of SU8 as a structural layer and how chemical additions have improved this.

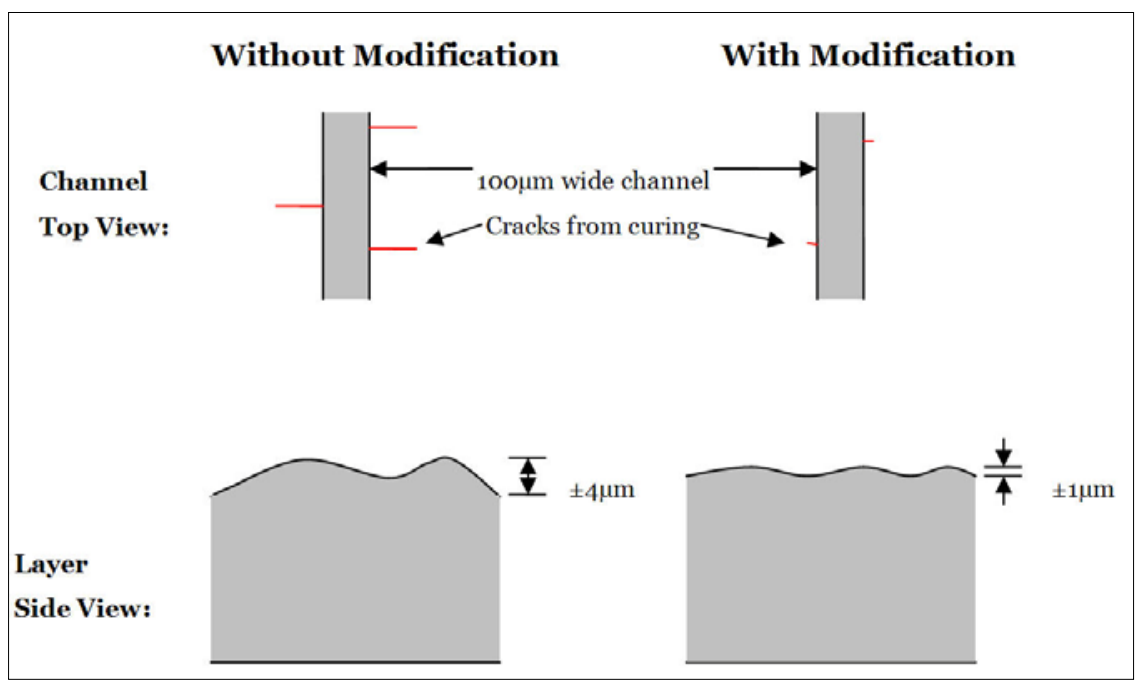

Figure 20. Modification of the SU8 formulation used to make individual microfluidic layers reduces both the number and size of the cracks emanating from the sides of the microfluidic channels by approximately a factor of 3 , and reduces the thickness variability of the layer by approximately a factor of 4 .

Switching the device construction material from PMMA to SU8 also enabled three important improvements:

1. Better dielectric coating (SU8 should have better electrical isolation ability than PMMA) to improve the sensor detection ability and reliability.

2. Lower device fabrication temperatures $\left(200^{\circ} \mathrm{C}\right.$ to $\left.90{ }^{\circ} \mathrm{C}\right)$ to enable the incorporation of metal electrode layers within a polymer stack without producing cracks in the metal. (Cracks formed in the metal layer due to high temperature processing and these are the leading cause of metal electrode failure.)

3. Better chemical inertness and stability of SU8 over PMMA.

\subsubsection{Initiative 3: Change in substrate carrier}

The third research initiative involved changing the temporary substrate carrier onto which each layer is fabricated before that layer is transferred to the device. The requirements of this material were to have (a) excellent solvent, acid, and base resistance to handle all the processing steps involved in the fabrication of each layer; (b) UV and optical transparency so that the layer could be aligned precisely and UV curing could be initiated 
through both sides; and (c) thermal stability at the maximum processing temperature $\left(\sim 200^{\circ} \mathrm{C}\right)$. Besides glass and glass compounds, there are very few materials that satisfied these criteria, but if the maximum processing temperature could be lowered, then a number of polymer epoxy based materials could be used. Because the first two initiatives were successful, new substrate materials are currently being investigated with the goal of matching the CTE of the device almost exactly (i.e., $\sim 55 \mathrm{ppm} /{ }^{\circ} \mathrm{C}$ ).

The problem encountered with using an all-polymer temporary substrate carrier is that the lower rigidity of the polymer, as compared to glass, requires the polymer layer to be thicker, but since polymers generally have lower thermal conductivity, as compared to glass, they also tend to bow when heated. All polymer carrier substrates have the benefit of producing unstressed layers on them, but the thickness uniformity of the layer suffers significantly as illustrated in Figure 21.

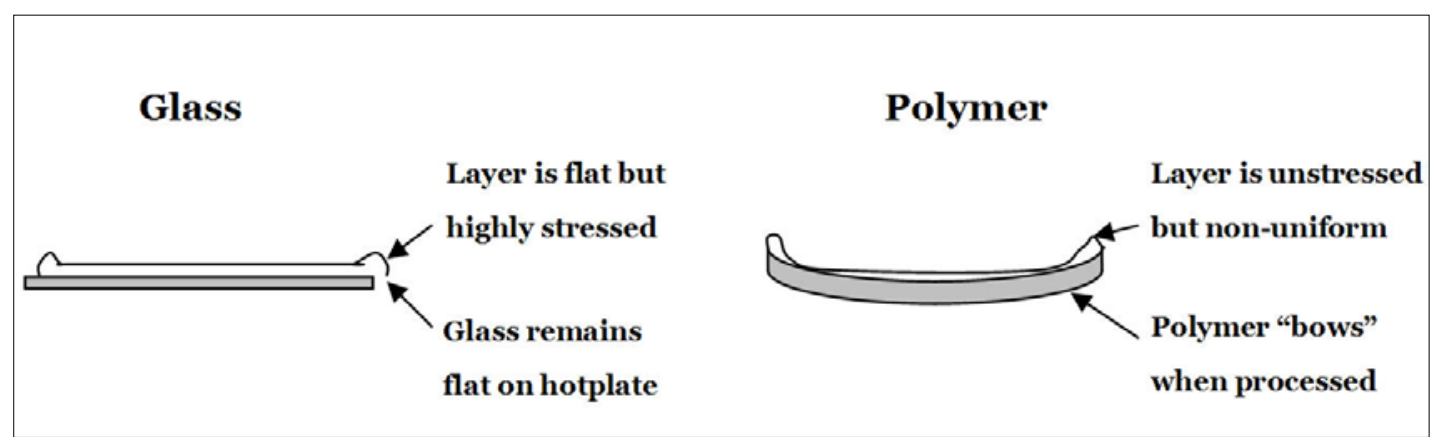

Figure 21. Choice of the temporary carrier substrate material has the tradeoff between forming a flat layer, although highly stressed on glass, or an unstressed layer but highly nonuniform on a polymer substrate. Because the polymer substrate has a lower rigidity than glass, it needed to be thicker, and since it was both thicker and had a lower thermal conductivity, it bowed during hotplate processing.

Composite polymer materials were then investigated to increase both the rigidity and thermal conductivity of the polymer temporary carrier substrate in an effort to eliminate bowing during the hotplate heating of the substrate. Adding glass particles to the polymer formulation seems promising. $\mathrm{As}_{\mathrm{SiO}_{2}}$ nanoparticles are added to some epoxy formulations, CTE is lowered, rigidity is increased, and thermal conductivity is increased (Shi, Zhou, and Edwards 2003; Zhou and Shi 2004). Complete results for this initiative are beyond the scope of this research. The addition of $\mathrm{SiO}_{2}$ nanoparticles appeared to help, but problems with keeping the $\mathrm{SiO}_{2}$ nanoparticles uniformly dispersed during the curing of the temporary substrate carriers are still problematic. Adding $\mathrm{SiO}_{2}$ nanoparticles to the layer material was also tried, but coating uniformity was problematic because it appeared 
that different surfactants, wetting agents, and de-foaming agents could be required.

The most promising approach by the end of this project appeared to be using a polymer with a CTE higher than the device (e.g., $\sim 70 \mathrm{ppm} /{ }^{\circ} \mathrm{C}$ ) and adding $\mathrm{SiO}_{2}$ nanoparticles to a weight percentage of $5 \%-15 \%$ such that the CTE of the substrate is matched to $\sim 55 \mathrm{ppm} /{ }^{\circ} \mathrm{C}$. Then almost all thermal stress will have been eliminated from the fabrication process and layers can be fabricated that are entirely crack-free and bonding will be completely unshifted.

Our final carrier construct was born out of the composite approach and consisted of using multiple materials to accomplish different functions, as illustrated in Figure 22. First, a rigid glass base ensured the carrier substrate would remain flat and heat relatively evenly. Second, a thin PDMS layer provided the stress-accommodating layer in the middle. Third, a thin SU8 layer was permanently attached to the PDMS layer to ensure that subsequent SU8 device layers would have a base onto which they would be CTE-matched.

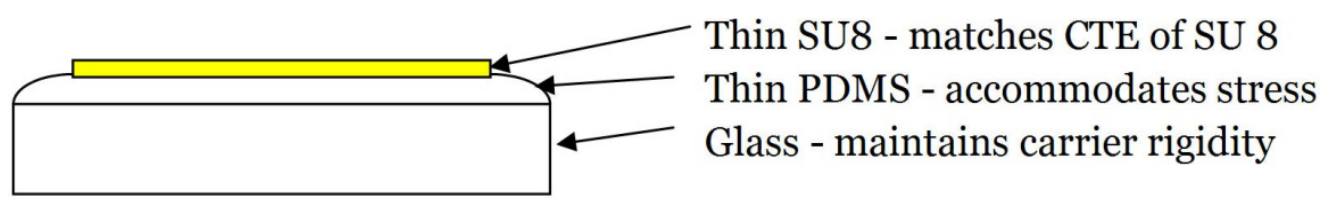

Figure 22: Current temporary substrate carrier construct consisting of a bottom rigid glass layer followed by a flexible stress accommodating PDMS layer and, on top, a rigid SU8 layer for CTE matching to SU8 device layers.

The carrier could then be used repeatedly to make stress-free SU8 layers as shown in Figure 23. First, a release layer was coated onto the carrier and cured, and then an SU8 layer was applied and patterned. Because SU8 has relatively low process temperatures, wrinkling can occur along the outer release layer but the SU8 base layer in the middle remains free of wrinkles and cracks, as evident by the microscope images of Figure 24. 


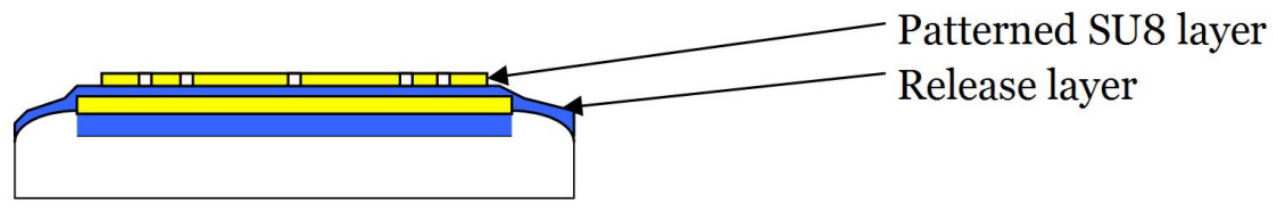

Figure 23. Current carrier substrate used to pattern a crack free SU8 layer where a release layer is coated first and cured, followed by the SU8 layer which is processed into a device layer.
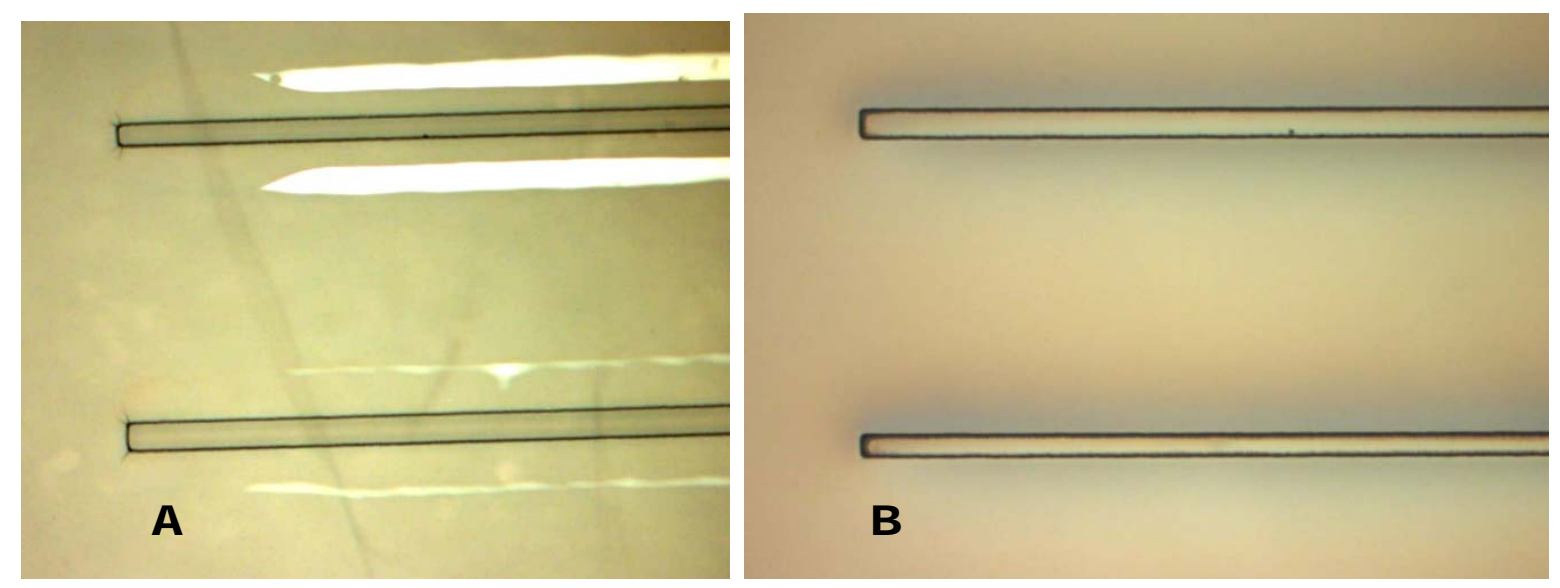

Figure 24. Microscope images of (A) cracks forming when SU8 is processed directly onto glass and $(B)$ a crack-free layer of SU8 when it is processed on our latest carrier substrate.

\subsubsection{Initiative 4: Improvement in bonding process for SU8}

SU8 is an inert material. This property results in excellent chemical stability but makes strong bonding between layers difficult to obtain. Experiments were undertaken to achieve SU8 surface epoxy bonds thus creating reactive end groups on the surface to improve adhesion. Approaches were used that included various RIE (Reactive Ion Etching) treatments and chemical treatments. Currently, the best approach appears to be a photografting technique using acrylic acid to modify the SU8 surface. This approach resulted in significantly stronger adhesive bonds between SU8 layers in experimental tests. In conclusion, the new temporary carrier substrates and devices made using the new adhesive process on these carriers have been fabricated, an example of which is shown in Figure 25. Challenges still remain concerning optimizing recipes and eliminating trapped bubbles during the bonding process, but critical problems regarding the addition of metal electrodes within polymer devices appear to have been resolved. Device testing is the next step. 


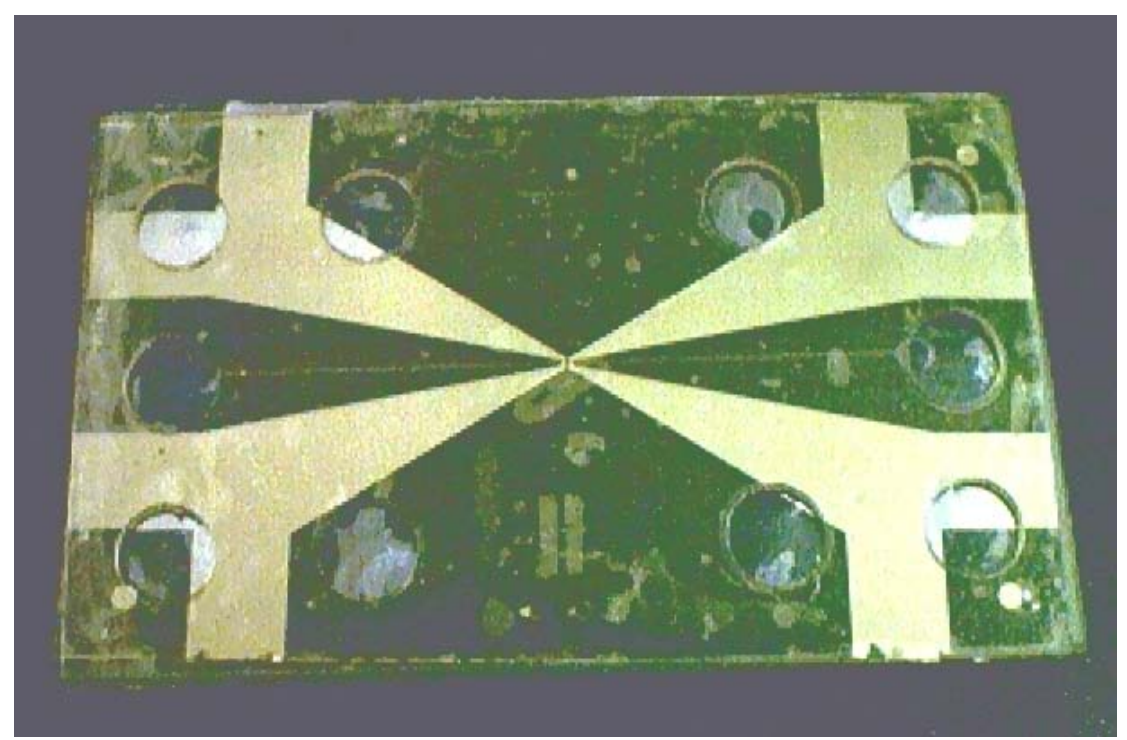

Figure 25. Example of a device made using SU8 and showing intact metal electrode layers.

\subsubsection{Initiative 5: Dielectric layer integrity}

For the capacitive electrodes to properly interrogate the microfluidic channel region, the integrity of the dielectric layer separating the metal electrode from the electrolyte in the channel must be maintained. In previous tests, the metal capacitor electrode is not sufficiently isolated from the channel, and a short is formed between the two. It is believed that very small "pinholes" (too small to see with a microscope) are formed in the polymer dielectric layers during the fabrication process which leads to an electrical short between the capacitor electrode and the microfluidic channel. An investigation of pinhole formation seems to correlate the size and depth of the pinholes to (1) thermal stress in the layer and (2) degree of crosslinking of the polymer. Thus, there are fewer problems with pinholes in polymers that exhibit higher crosslinking and in polymers that have been processed at lower temperatures. Figure 26 illustrates these principles. 


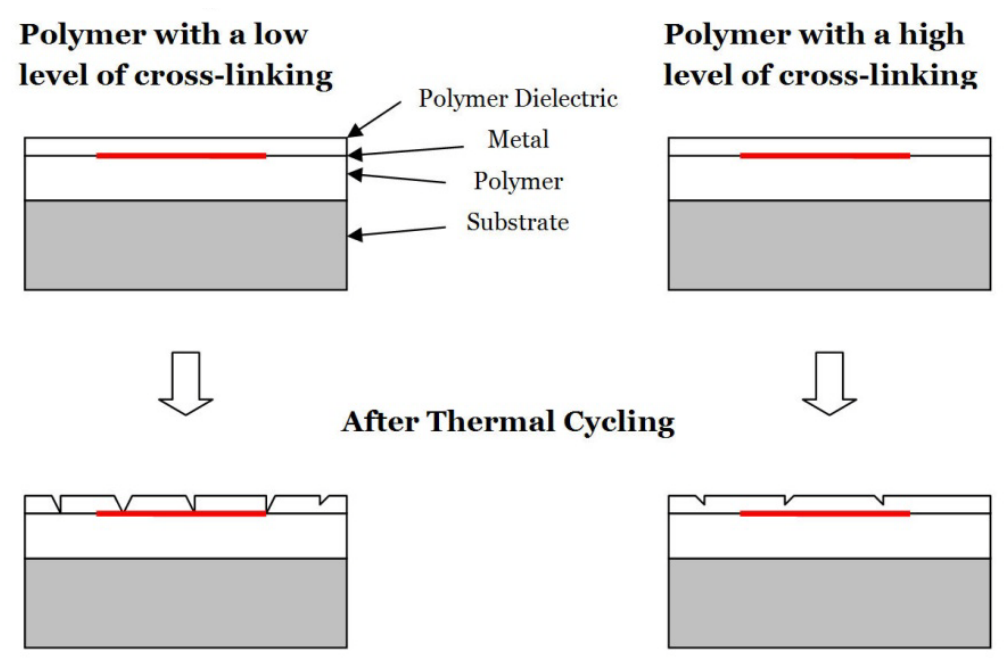

Figure 26. Polymers with a higher level of cross-linking appeared to have fewer pinholes, or pinholes of shorter depth; greater thermal stress on lower cross-linked polymers also seems to increase pinhole depth and density.

The research initiatives that reduced both the maximum temperature used during fabrication and implemented polymer materials with a higher density of crosslinking have allowed polymer dielectric layers with greater integrity. This should enable thinner polymer dielectric layers in the future and thus make the capacitive interrogation of the channel region that much more sensitive.

Radically new fabrication processes and materials have been developed as a product of this research. At the beginning of this project, it was not foreseen that to fabricate the intended device it would require (a) a new adhesive, (b) a new layer material, or (c) a new dielectric understanding and development.

\subsubsection{Increased device failure rate: cause and effect}

We used the established adhesive contact printing procedure to fabricate the hybrid nanofluidic / microfluidic devices in the clean-room. Our multilayer hybrid polymethyl methacrylate (PMMA) / polycarbonate membrane devices had a marked decrease in operating performance before failure. For example, a fabricated device was stable for two days before it failed on the third day. After drying the device over the weekend, it appeared to be functional; however, the separation was not reproducible. Then the device failed permanently a short time after it was operated for amino acids separation using $20 \mathrm{mM}$ Borate buffer, $\mathrm{pH}$ 9.3. When the device failed, the migration times became random, and data from the device was meaningless. 
Basically, the devices delaminated after one or two days of operation, although we used the same procedure as previously used. Significant tests were performed to investigation fabrication parameters including bonding temperature, bonding pressure, adhesive thickness, and curing duration. However, all the devices fabricated with different conditions had poor durability in separation applications. Finally, we noticed that the adhesive components of solid epoxy ( 24 months of shelf time) and its hardener (12 months of shelf time) had expired. Unfortunately, Dow Chemical Co. no longer stocked those materials. To solve this problem, we increased the percentage of hardener in the adhesive composition to compensate for the reduced active hardener in our older mixture. This new device worked better. However, after about $20 \mathrm{~h}$ of operation, the device failed. The intersection between the separation channel and the injection channel started to delaminate. The delaminated width was $\sim 50-60 \mu \mathrm{m}$ on both sides of the two channels. However, the far end from the injection point of the separation channel seemed to be fine. Considering that the previous devices had delaminated along the entire channel, increasing the hardener amount was an improvement in terms of device durability.

\subsection{Separation and detection of amino acids}

This aspect of the project describes the interfacing of two sequential injections to create multi-dimensional separations before detection. This will enable more complex samples to be fractionated before being introduced to the DNAzymes.

To develop an integrated microfluidic device for separating, sensing, and quantifying a complex analytical sample, we conducted research to demonstrate the sequential manipulation on tiny volumes of solution using a three-dimensional hybrid nano/ microfluidic device. Specifically, a multi-layered microfluidic separation system was fabricated using a PMMA substrate. Then, a PCTE membrane was sandwiched between the two PMMA microfluidic layers and acted as a fluidic gate via its array of nanometer diameter pores (the nanocapillary array).

The fluidic gate could be "opened" or "closed" by applying appropriate bias voltages to control the transport of analytes between crossed microfluidic channels. Analytes injected into a separation channel thus could be "captured" by opening the gate and transferring the analytes into a second, vertically displaced, microfluidic channel. 
A mixture of fluorescein isothiocyanate (FITC) labeled amino acids was analyzed to demonstrate application of this integrated microfluidic/ nanofluidic PMMA separation system. Two such amino acids-D-/ Laspartate and D-/ L-serine - are amino acids that may have different functions depending on the particular chiral form in the brain. Thus, separation of these two amino acids served as a model system to characterize overall system performance.

The amino acids separated during the first stage separation were collected by passing them through the nanocapillary array and, in this case, the coeluting enantiomeric pair was then separated using a chiral, second-stage electrophoretic separation. In our system, analytes were detected using laser induced fluorescence (LIF).

Figure 27 gives the design and dimensions of the PMMA microfluidic device. Figure 28 describes the separation scheme. Figure 29 represents the experimental setup, in which split laser beams focus at two detection spots, LIF1 and LIF2, and fluorescence signal was detected using two PMT detectors.

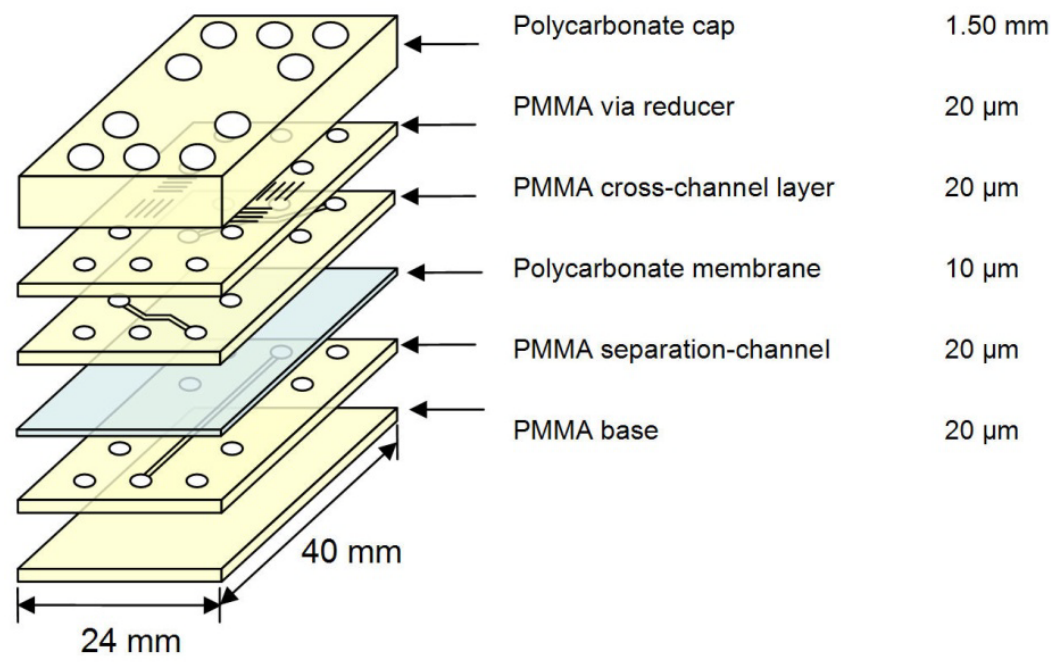

Figure 27. A schematic drawing of a PMMA multi-layer microfluidic device with associated thickness of each layer of the device. 


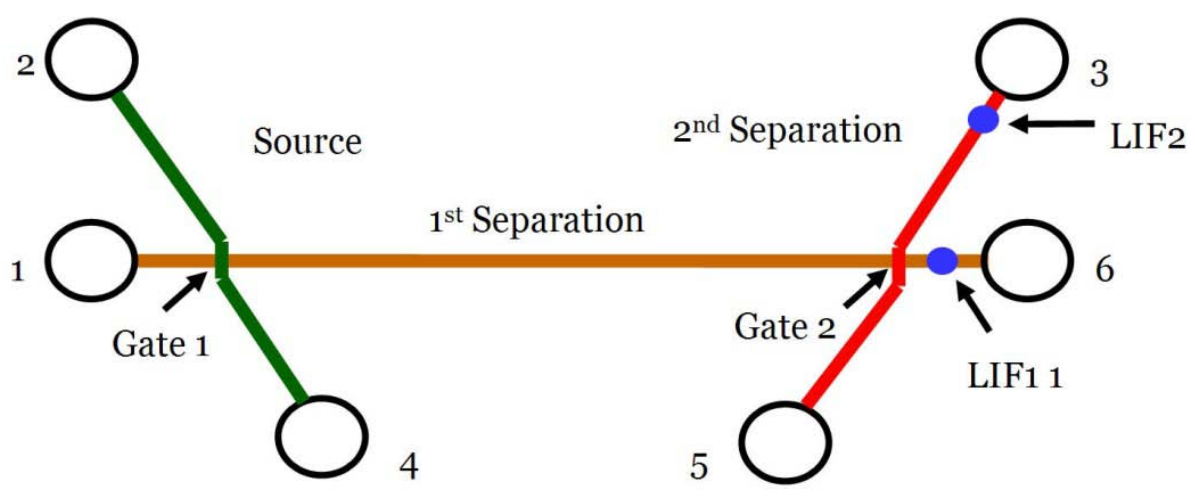

Gate: $220 \mathrm{~nm}$ nanocapillary array

Figure 28. Separation scheme.

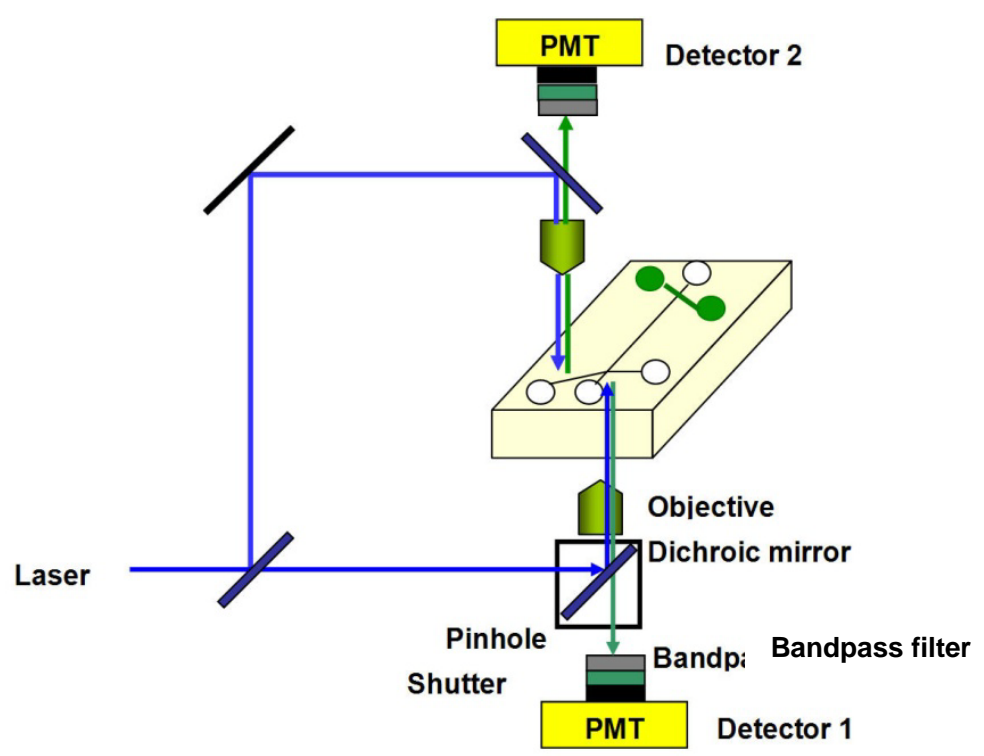

Figure 29. Conceptual sketch of the experimental setup.

Manipulation of analytes through nanoscale fluidic molecular gates is achieved by external control of applied potential bias. Voltage configuration at different steps is outlined in Figure 30. 


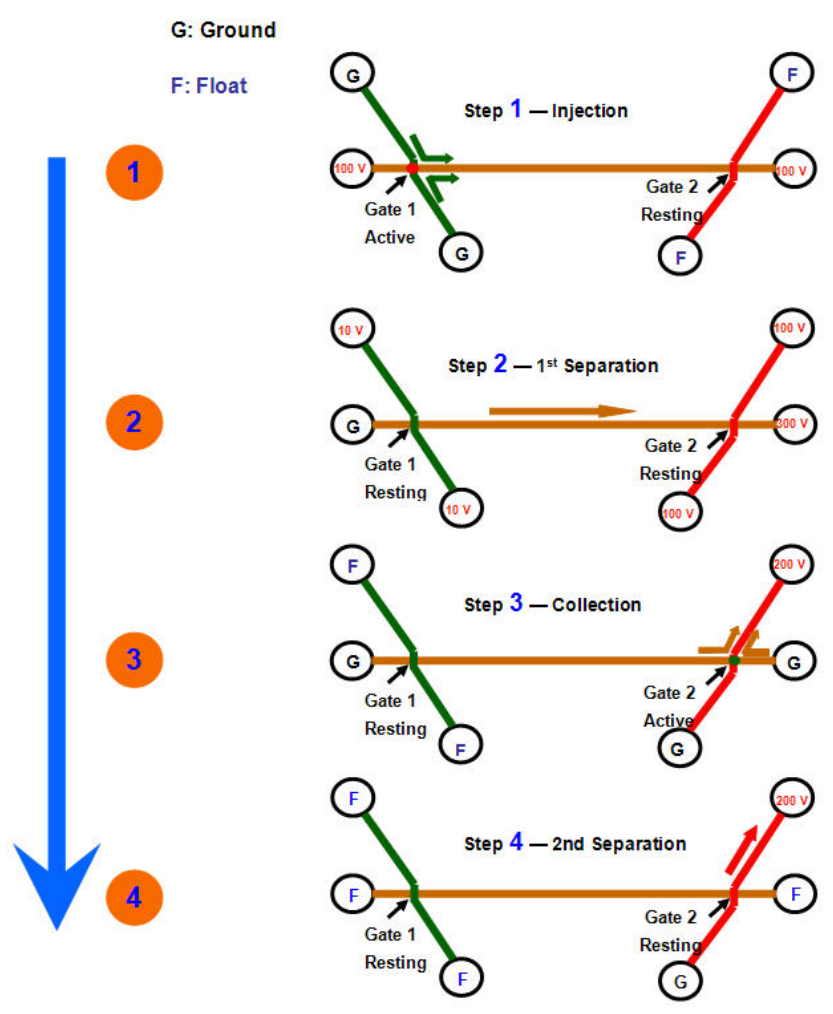

Figure 30. Voltage configuration.

Reproducible injections and separations were obtained for FITC labeled D-/ L-aspartate and D-/ L-serine. According to the migration time of different molecules, analytes of interest can be collected by adjusting the starting collection time. Both figures below show three repeated injections of aspartate and serine (Figure 31 and Figure 32). Collection of analytes was disabled during the first two injections in both experiments. Figure 31 shows the collection of the aspartate peak at gate 2 for the third injection, which was confirmed by the disappearance of this peak in the detection spot LIF 2 (red trace). Similar results with selective collection of the serine peak for the third injection are shown in Figure 32. In both experiments, the collection time was set for $5 \mathrm{~s}$. 


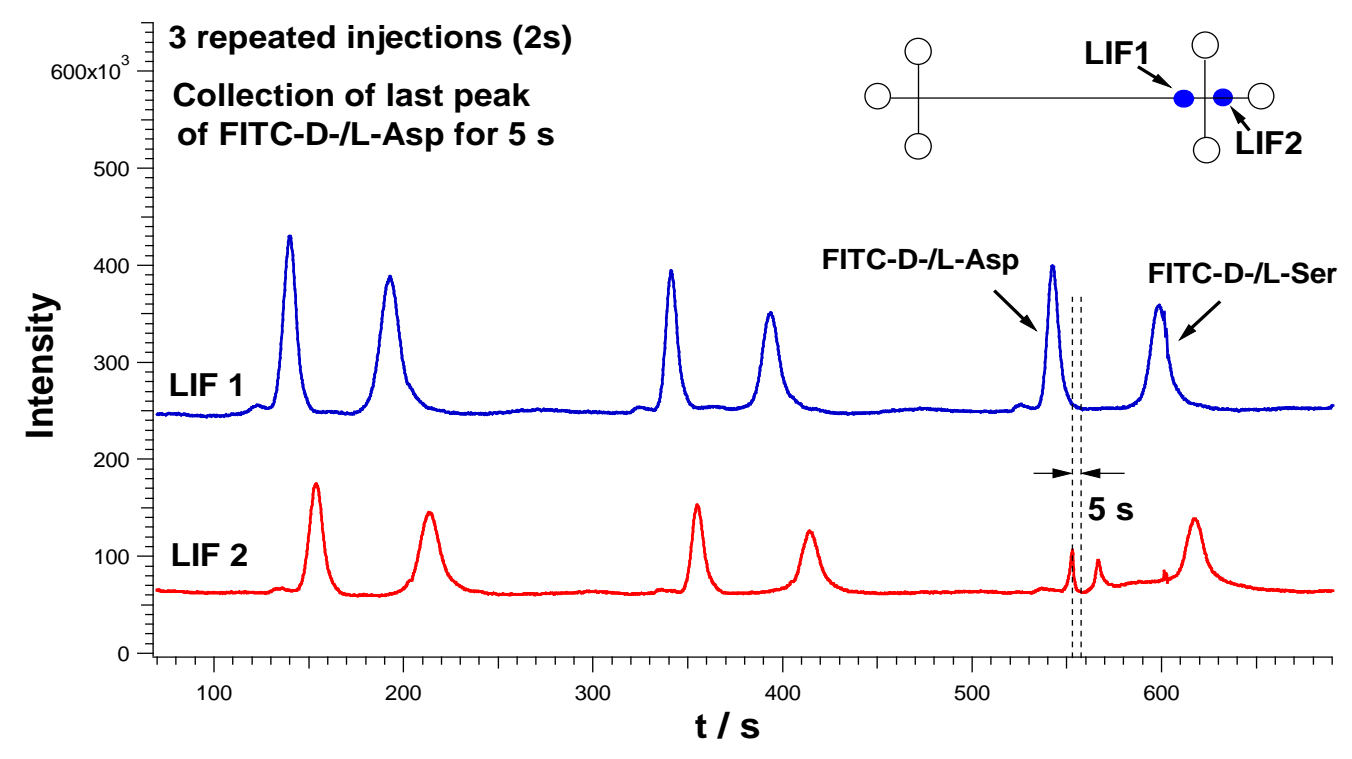

Figure 31. Collection of FITC-labeled aspartate for the third injection.

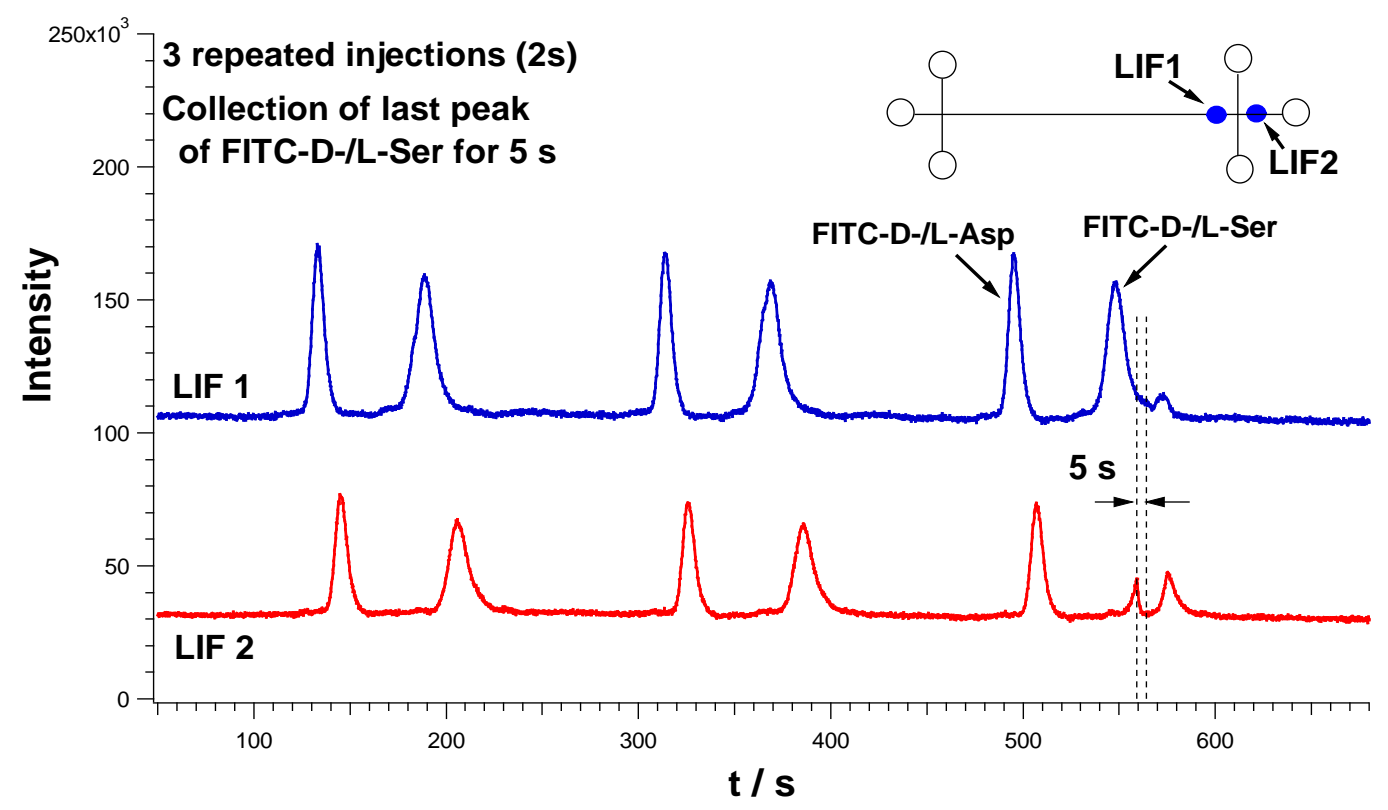

Figure 32. Collection of FITC-labeled serine for the third injection. 


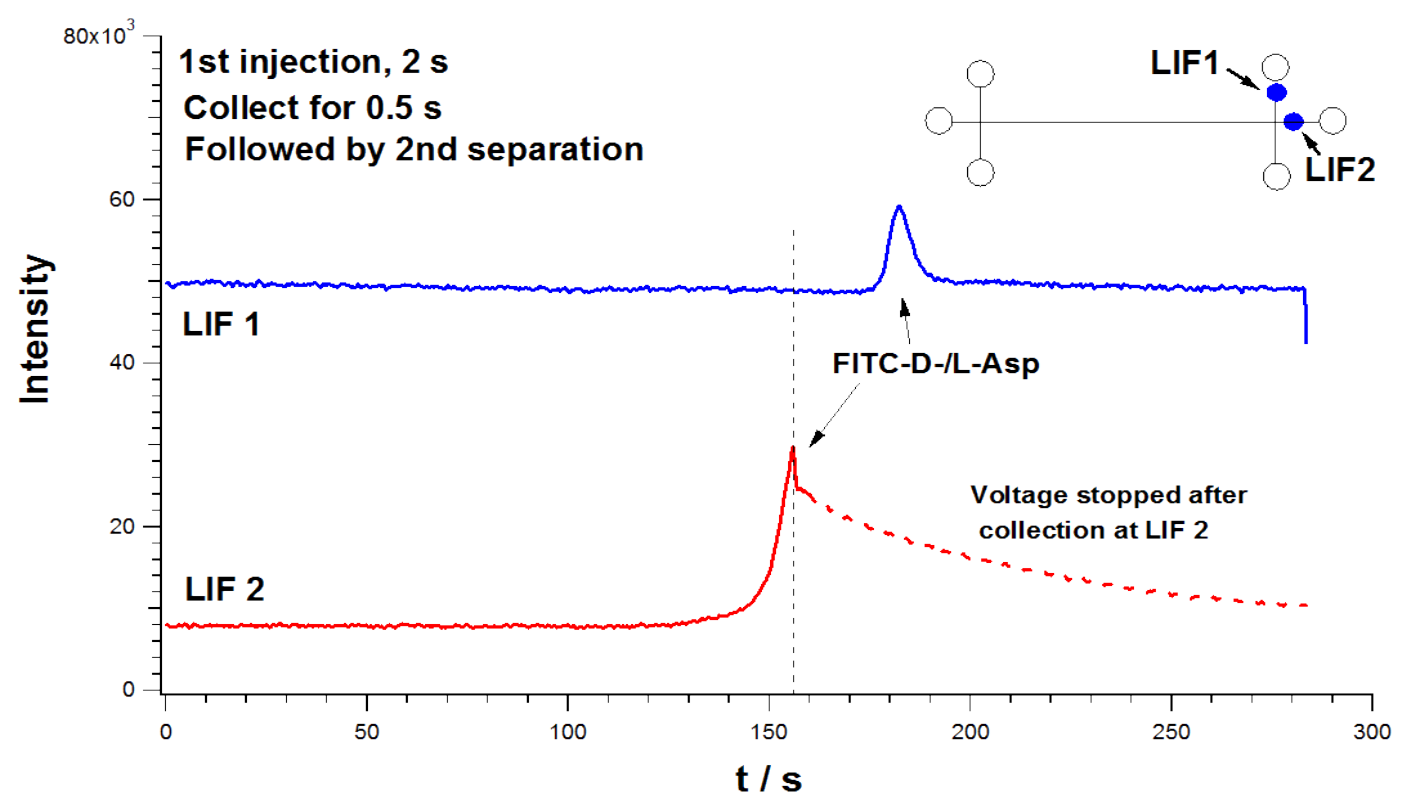

Figure 33. Demonstration of collected peak of aspartate.

When one of the detection spots was set at the second vertical channel, collection of analytes of interest was clearly demonstrated (Figure 33). The collection time was $0.5 \mathrm{~s}$ for aspartate in this example. When we noted the presence of aspartate at LIF2 (red trace), we injected the stream into the detection channel, whereupon a small peak of aspartate (blue trace) was observed. This illustrates the ability to selectively move fluid flow from one channel to another by merely altering the voltages applied at the reservoirs.

Injection, separation, and collection of analytes were achieved successfully using our three-dimensional PMMA microfluidic device. The next step was to perform chiral separation in the second vertical channel after collection of analytes. Integration of multiple fluidic operations into a single microfluidic device allowed more complex manipulations to aid in solving complex analytical problems. The second-stage operation is not limited to a separation as it can be used for mixing, sensing, reacting, or analyte concentration.

In the demonstration below, the exemplary analytes were fluorescently tagged chiral amino acids (Table 4), to demonstrate the ability to perform difficult multidimensional separations. 
Table 4. Analytes used for testing.

\begin{tabular}{|l|l|l|l|l|l|l|}
\hline \multirow{2}{*}{ Name } & \multirow{2}{*}{$\begin{array}{l}\text { Abbrev. / } \\
\text { MW }\end{array}$} & $\begin{array}{l}\text { Carboxylic } \\
\text { acid }\end{array}$ & Amine & pl & $\begin{array}{l}\text { Functional } \\
\text { Group, pKa }\end{array}$ & $\begin{array}{l}\text { Sep. } \\
\text { Order }\end{array}$ \\
\hline Aspartic Acid D & Asp / 133.1 & 2.1 & 9.82 & 2.8 & $\begin{array}{l}\text { carboxylic } \\
\text { acid 3.86 }\end{array}$ & 1 \\
\hline Glycine G & Gly / 75.07 & 2.35 & 9.78 & 6.0 & none & 2 \\
\hline Serine S & Ser / 105.09 & 2.21 & 9.15 & 5.7 & hydroxyl & 3 \\
\hline Asparagine N & Asn / 132.12 & 2.02 & 8.8 & 5.4 & amide & 4 \\
\hline *Phenylalanine F & Phe / 165.19 & 2.58 & 9.24 & 5.5 & aromatic & 5 \\
\hline
\end{tabular}

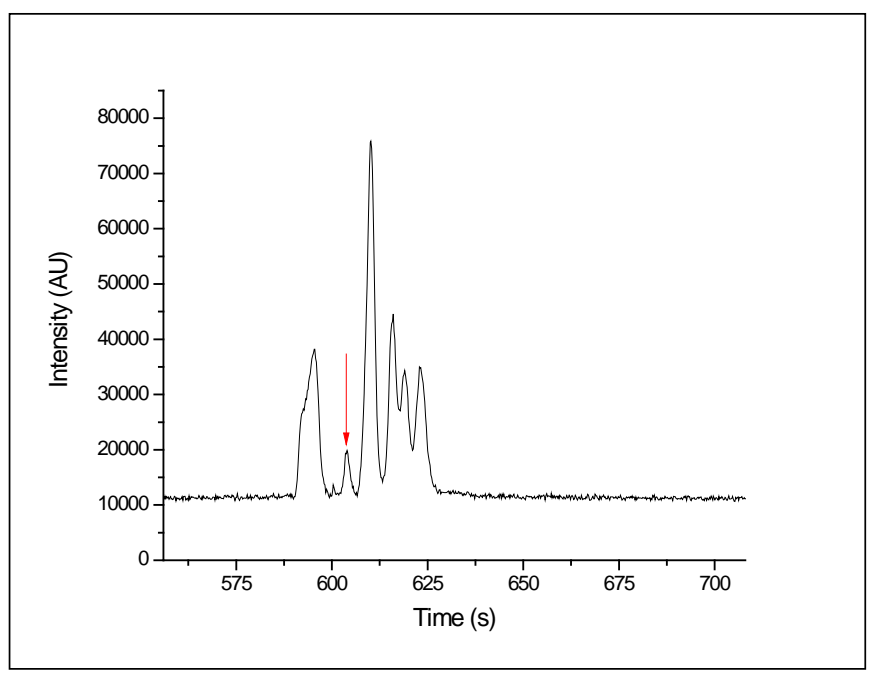

Figure 34. Separation of 5 amino acids in $20 \mathrm{mM}$ borate buffer $\mathrm{pH}$ 9.3. The electropherogram was from a series of multiple injections. The maximum peak intensity of the first peak was observed in $39 \mathrm{~s}$ after the second injection. The peak marked with the arrow on the electropherogram is an unknown impurity. The separation orders of the analyte peaks are assigned as shown in Table 4.

This newer device performed differently by showing significantly smaller injection volumes than previous devices although analyte migration times were roughly similar (or faster). In order to increase the injected amount of the analyte, we added $30 \mu \mathrm{M}$ cetyl trimethylammonium bromide 
(CTAB) in the $20 \mathrm{mM}$ borate buffer ( $\mathrm{pH}$ 9.3). Adding CTAB increased the amount of analyte injection; however, the 5 analyte peaks did not separate. The buffer was changed to $20 \mathrm{mM}$ borate buffer ( $\mathrm{pH}$ 9.3) without CTAB for improved separation performance. Now the analyte peaks were separated but the injected amount was less, compromising signal-to-noise ratio as well as chromatographic resolution (Figure 35). With our chiral separation buffer ( $20 \mathrm{mM}$ borate $\mathrm{pH}$ 9.3; $18 \mathrm{mM} \beta$-cyclodextrin [ $\beta$-CD]; $6 \mathrm{mM}$ sodium taurocholate [STC]), no analyte peaks were observed (ideally, 8 peaks should be separated and detected). After $30 \mu \mathrm{M} \mathrm{CTAB}$ was added to the buffer, and the analyte injection peak was observed at the cross section of the source and the separation channels. However, extended operation of the device under these conditions caused delamination. We solved this problem by using the new adhesives described in this report.

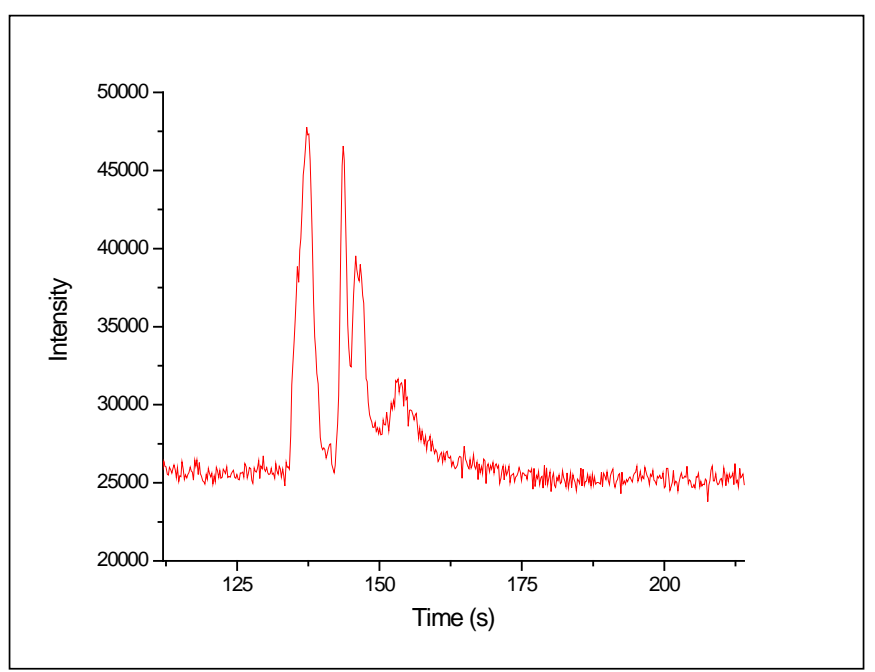

Figure 35. Electropherogram collected with the new test device. The electropherogram was from a series of multiple injections. The first peak showed maximum intensity after $25 \mathrm{~s}$.

\subsubsection{Chiral separations}

This section describes our progress toward true multidimensional separations. The goal was to take a selected band from a separation, inject it into a distinct microfluidic layer with a different electrolyte, separate the single band into its components, and detect them. Thus, the peak capacity and chance of false identification is greatly decreased, especially when the electrophoretic separations in the two layers use different separation mechanisms such as free zone electrophoresis and micellar electrokinetic chromatography. 
Using solutions defined as (A) $50 \mathrm{mM}$ borate buffer $\mathrm{pH}$ 9.3; (B) $6 \mathrm{mM}$ sodium taurocholate and $18 \mathrm{mM} \beta$-cyclodextrin in (A); and (C) $1 \mathrm{mM} \mathrm{CTAB}$ in DI water, two additional solutions were made (D) A:MeOH =4:1(v/v); and (E) B:MeOH =4:1 (v/v). With the device configuration as shown in Figure 36, the first stage separation used a running buffer of (C):(D) = 25:75 (v/v) (final - $25 \mu \mathrm{M}$ CTAB in solution), while the second stage separation used a chiral buffer of $(\mathrm{C}):(\mathrm{E})=25: 75$ (v/v) (final - $25 \mu \mathrm{M}$ CTAB in solution). The analytes injected are $1 \mathrm{mM}$ labeled "D/ L-Asp" and "D/ LSer." Data from Figure 36 show the fluorescent signals from the separations of these labeled amino acids. The bottom trace (left) is the separation of the Asp from the Ser in the first dimension. We then selectively extracted the Asp for injection into the second-stage chiral separation which leads to the upper trace. The two enantiomers of Asp are clearly separated to near baseline resolution. This demonstrates precise control of fluid flows and separation parameters that will allow enhancement of selectivity for the final device.
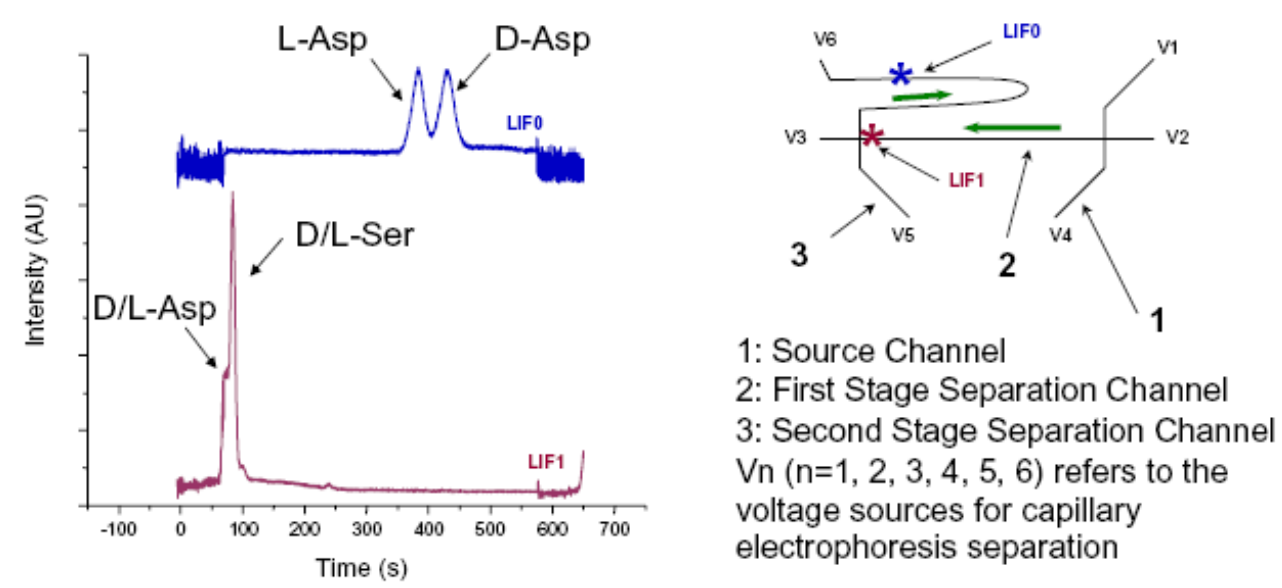

Figure 36. Multi-dimensional separation on a PMMA hybrid microfluidic/nanofluidic device. LIF1 is the first stage separation data and LIFO is the second stage separation data.

\subsection{Microfluidic devices with Au-coated NCAMs}

Work thus far had focused on understanding fluid transport in devices with Au-coated NCAMs. The principal goal of this portion of the project was to evaluate the possibility of using Au-coated NCAMs within a microfluidic device to effect DNAzyme immobilization and subsequent sensing of $\mathrm{Pb}^{2+}$.

Incorporation of Au structures into microfluidic devices raises several issues for fluid handling. Typically, fluids are electrokinetically driven both 
in microfluidic channels and across the nanopores. Employed field strengths are on the order of $10-100 \mathrm{~V} \mathrm{~cm}^{-1}$ for microchannels and 100 $1000 \mathrm{~V} \mathrm{~cm}^{-1}$ for nanopore membranes. However, the low resistivity of $\mathrm{Au}$ means that it can sustain only small relative end-to-end potential differences under the applied voltage conditions. However, given the large microchannel voltages typically employed, it is not difficult to achieve conditions where the end-to-end potential difference exceeds the width of the ideally polarized region in the aqueous electrolyte, meaning that the potential gradient established can lead to various redox processes along the length coordinate of the Au region, as illustrated in Figure 37. These, in turn, lead to anodic and cathodic processes that are balanced (i.e., no net charging of Au occurs).

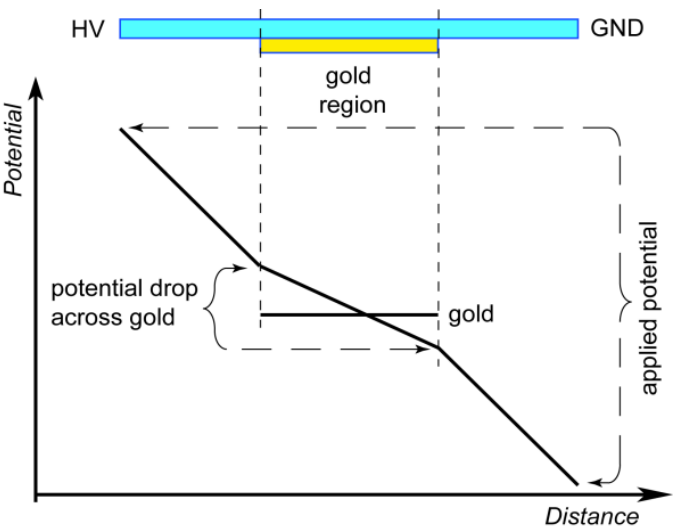

Figure 37. Schematic diagram of potential distribution along a microfluidic channel in contact with a gold membrane. The illustration at the top of this figure represents one microfluidic channel and the Au-NCAM in cross-section. The electric field in the microfluidic channel, $\mathrm{dV} / \mathrm{dx}$, is smaller above the Au membrane due to the high conductance through the Au.

The potential drop across the Au region was determined by a combination of the ohmic resistance of the microfluidic channel and faradaic processes occurring at the gold-solvent interface. Since the potential difference that causes electrochemical solvent (water) breakdown is the largest accessible potential difference, this greatly reduces the electric field strength accessible for liquid transport directly above the $\mathrm{Au}$. In order to reduce the effect of the Au, microfluidic devices with NCAMs coated only at the intersection of microfluidic channels were fabricated. Fabrication details and a bright field micrograph of a channel intersection are shown in Figure 38. 


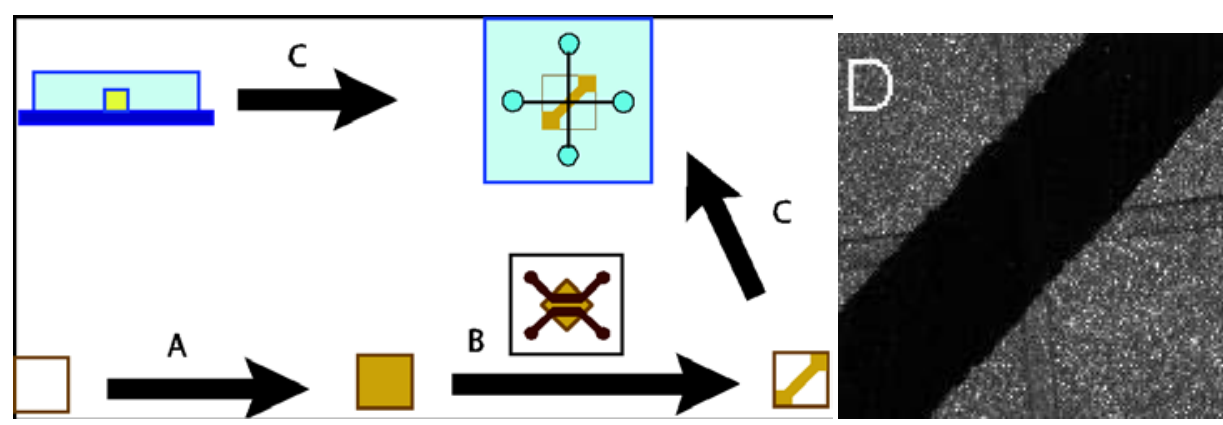

Figure 38. Fabrication details. A: electroless deposition; B: patterning of NCAM using $\mathrm{KI} / \mathrm{I}_{2}$ etchant; C: assembling PDMS device using rapid prototyping; D: brightfield micrograph of microfluidic device at intersection of microfluidic channels; the Au-coated region appears as a black region in the image. Width of channel and gold feature are 50 and $280 \mu \mathrm{m}$, respectively.

Microfluidic devices consisting of two channels created narrow gold features using $\mathrm{KI} / \mathrm{I}_{2}$ etching. The gap between the microfluidic channels constitutes the remaining gold feature on the NCAM. The width of the gap was $280 \mu \mathrm{m}$, the width of channel was 700 or $1500 \mu \mathrm{m}$. The device was fabricated using standard techniques with pattern height of $50 \mu \mathrm{m}$. Gold coated NCAM (PC, $400 \mathrm{~nm}$ pore diameter) was placed on a PDMS slab and a second PDMS piece with a channel pattern was placed on top of the NCAM. $\mathrm{KI} / \mathrm{I}_{2}$ etching solution was introduced in both channels and replaced every few minutes. The etching solution penetrates through pores and etches gold away from both sides of NCAM. The end of etching is easily observable by color changes in the channel: dark metallic to light yellow. The remaining gold (on either side of the narrow feature) is removed in an additional step and the narrow gold feature is protected by two pieces of PDMS.

The devices with wider etching channels $(1500 \mu \mathrm{m})$ were more convenient. The channel width is small enough that channel does not collapse, but wide enough to make the second etching step easy to perform. This method is much more practical than direct gold deposition in the microfluidic device. Gold deposition in microfluidics requires substantially more control (temperature, careful washing between different reagents, aligning of two channels, etc). The inherent hydrophobicity of PDMS prevents etching solution leakage between the two channels.

These devices are directly useful up to electric fields $\mathrm{E} \leq 80 \mathrm{~V} / \mathrm{cm}$; however, removing gold from the exposed surfaces of NCAMs (Au only in the 
nanochannels; no continuous Au film exposed to microchannel) significantly increased the accessible field strength above $200 \mathrm{~V} / \mathrm{cm}$.

The obvious failure mechanism of these devices is gas bubble nucleation. Physically, the two ends of the Au electrode sustain a driving potential for electron transfer, oxidation at one end, reduction at the other, across the solution-Au interface to sustain current in the microfluidic channel. Because gas generation has both thermodynamic (local potentials at the $\mathrm{Au}$ edges) and kinetic (nucleation rate vs. gas diffusion) components, we sought to understand these by comparing experimental observations to models. Gas (hydrogen and oxygen) evolution at the edges of the Au region was investigated in light of a published theoretical model. The underlying assumptions of the model are: (a) linear potential drop across the electrode, (b) current determined by the Butler-Volmer equation (electron transfer is kinetically limiting), and (c) anodic and cathodic currents are equal in magnitude (no charging of electrode occurs). Anodic and cathodic processes, and their standard potentials and kinetic parameters (transfer coefficient and exchange current) are known. However, within the constraints of the model, current and potential can be calculated along the spatial axis of the electrode. An example of a current density calculation is given in Figure 39, illustrating that, consistent with experimental observation, the redox processes are dominated by the electrode edges. Current density strongly varies with applied potential, but its distribution is essentially invariant. Anodic (cathodic) processes on gold occur on the side that faces the device cathode (anode). The theoretical potential difference to generate gas bubbles in the microfluidic channel is somewhat larger than experimentally observed ( 1.6 to $2.6 \mathrm{~V}$ ). Due to limitations of the model, however, the agreement between predicted and experimental is satisfactory.

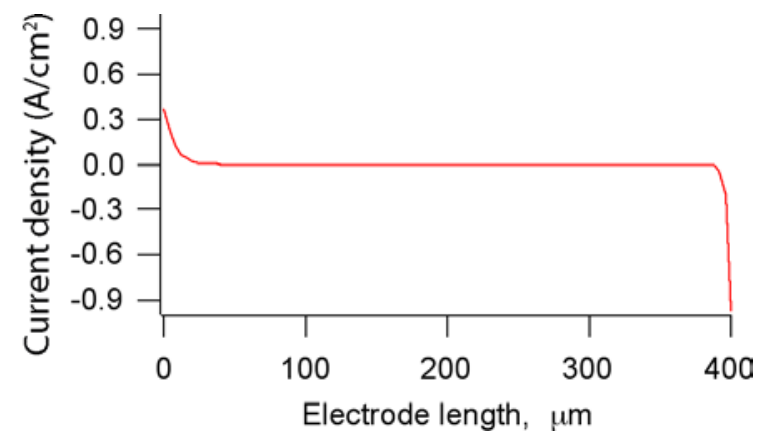

Figure 39. Current distribution on gold, $\Delta V \sim 3 \mathrm{~V}$. Cathodic current is represented as a positive current. 


\subsubsection{Reducing the effect of Au coating}

A typical fabricated device has an Au contact length on the order of 250 to $300 \mu \mathrm{m}$ which is a compromise between the ability to align gold features at the intersection of microfluidic channels and minimal adverse effects due to Au presence in a device. We have looked at 3 different Au-coated NCAM architectures (Figure 40): (1) NCAM coated with gold within nanopores and on sides exposed to the microchannels; (2) NCAMs coated only on one side; (3) NCAMs coated with gold island film (nanometersized gold regions that are not electrically connected). Microfluidic devices consisting of two channels with an Au-coated NCAM sandwiched in between two microfluidic channels were constructed as shown in Figure 38. Type (1) and (3) membranes were obtained by electroless deposition of $\mathrm{Au}$ and subsequent etch using $\mathrm{KI} / \mathrm{I}_{2}$. Continuous gold films (type 1) are obtained if deposition is performed for 1-5 h; and gold island films (type 3) are obtained if deposition times are kept between 15 and $30 \mathrm{~min}$. Gold vacuum evaporation was used to obtain NCAMs coated with Au only on one side (type 2 , width of gold feature $\sim 250 \mu \mathrm{m}$ ). The arrows show the ability to electrokinetically transport species through the nanochannels.
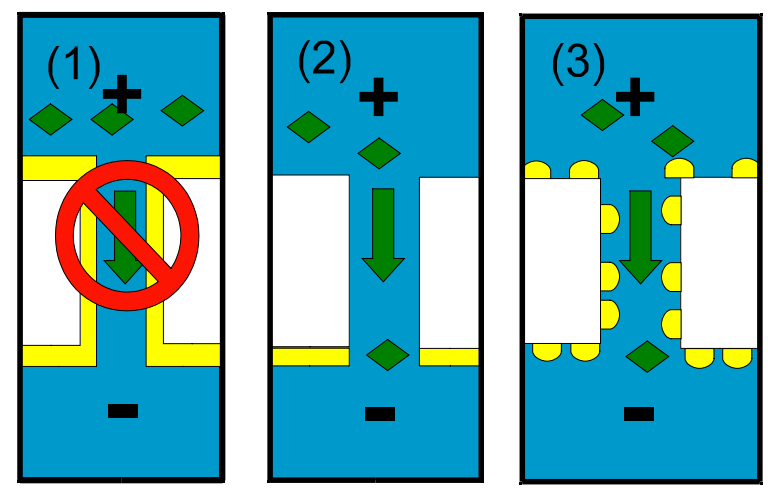

Figure 40. Au-coated NCAM architectures: (1) continuously coated; (2) coated on one side; (3) gold island film coated NCAMs. Mathematical signs indicate applied potential across the membrane, and arrows indicate the direction of electrokinetic transport.

The presence of Au on the NCAM complicates analyte injection across the NCAMs. Typically, NCAMs have Au coated on the NCAM faces exposed to microfluidic flow as well as in the nanopores. Injection of fluorescein across these NCAMs is suppressed. Simultaneously, transport of fluorescein away from the intersection of the microfluidic channels is observed. Only analyte directly within the cross-section of the microfluidic channels and, therefore, close to the entrance of a nanopore is transported across the NCAM to the trans microchannel. Examples of injections across NCAMs are illustrated on Figure 41. Considerably better injections are 
achieved when gold is not present on the large surfaces exposed to microchannel flow.

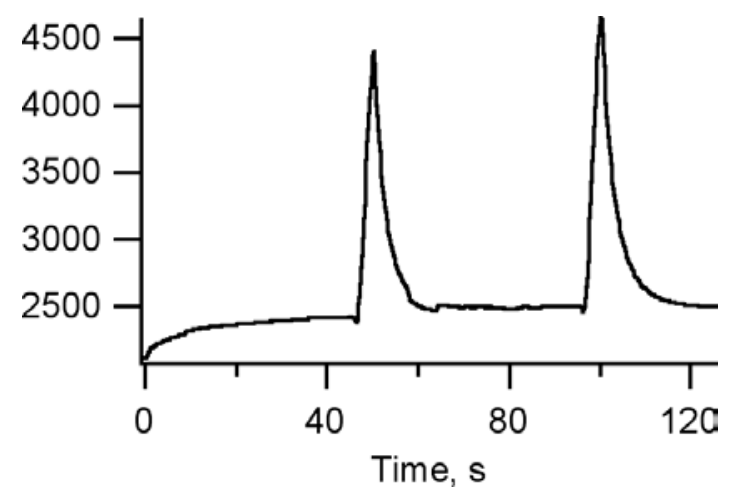

Figure 41. Injection across gold coated 400 nm NCAMs.

\subsubsection{Applications of NCAMs in affinity-based separations}

A research goal was to evaluate the potential and possibilities for placing recognition molecules within the attoliter volumes of the nanopores to approach unit probabilities for reactions and interactions.

One of the key questions that has to be answered to effectively deploy molecular recognition moieties (such as the $\mathrm{Pb}^{2+}$-sensitive DNAzyme molecular beacon construct) surrounds the changes in reactivity that attend immobilization of the reagent on the interior wall of a nanopore. We anticipated that ligand binding in nanometer-scale spaces would be determined by two countervailing tendencies - the increase in wall-collision frequency, $\Omega \mathrm{w}$, of prospective ligands (e.g., $\mathrm{Pb}^{2+}$ ) and the reduced volume of phase space explored due to hindered rotational diffusion, $D_{R}$, inside the pore. Our work addressed three specific technical objectives: (a) to develop robust and flexible fabrication strategies for the immobilization of molecular recognition motifs on the interior surfaces of individual nanocapillaries; (b) to create and implement assays for ligand binding which are sensitive to the intrapore binding events; and (c) to assess whether the ligand-affinity reagent complexes can be released with sufficient control to form the basis for high efficiency nanoscale affinity separations.

NCAMs consisting of thin $(5 \mu \mathrm{m}<\mathrm{d}<20 \mu \mathrm{m})$ membranes that contain arrays of nanometer-diameter $(10 \mathrm{~nm}<\mathrm{a}<500 \mathrm{~nm}$ ) pores are used in the SERDP device to interface vertically separated microchannels and allow the external control of analyte transport between microfluidic channels. In 
order to add functionality to these nanopores beyond simple fluid transport, we have incorporated an antibody-based molecular recognition element onto the pore surface that allows selective capture, purification, and release of specific analyte(s) from a mixture as our proof of principle. The pores are fabricated by electroless plating of gold onto the nanopores of an NCAM (Au-NCAM). An antibody is then immobilized within the AuNCAM via gold-thiol chemistry as a thiolated fragment-of-antibody (Fab), as shown in Figure 42. The successful immobilization of the resultant thiol-terminated HS-Fab' through this protocol was verified on planar gold by its biological activity and by fluorescence microscopy, scanning electron microscopy and atomic force microscopy.

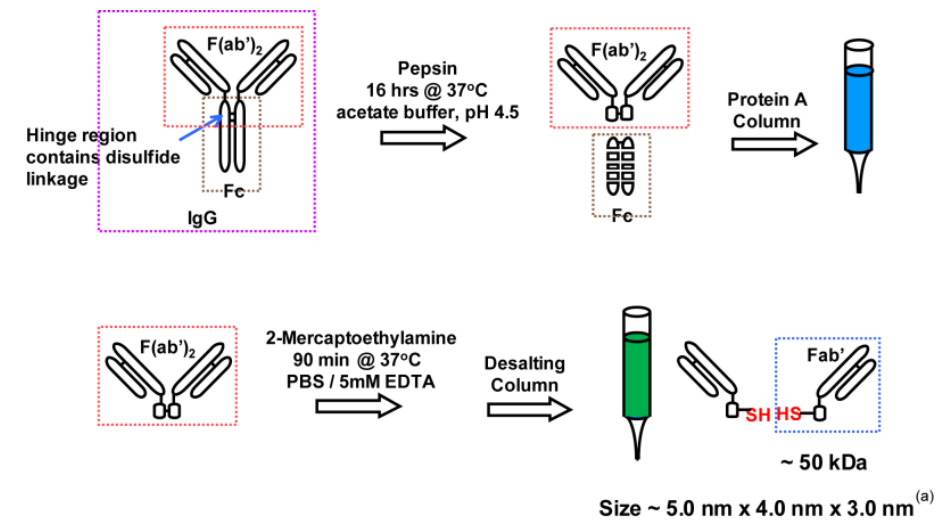

Figure 42. Immobilization of Fab'.

The next question we addressed focused on immobilizing the Fab' fragments on the interior of nanopores of Au-coated NCAMs (Fab'-Au-NCAM) prepared by electroless Au deposition. To do this, we performed a goldlabeled sandwich assay as shown in Figure 43. The presence of biochemically active Fab' is signaled by the binding of a Au-labeled IgG which is evident in the enhanced roughness of the nodules in the freeze-fracture SEM in panel (A) compared to those in (C) and (D). The importance of these experiments is that they illustrate (a) successful binding of Fab' fragments onto the Au-coated nanopore wall using Au-thiol chemistry, and (b) the bound Fab' fragments retain their biochemical function under these conditions. 
(A)

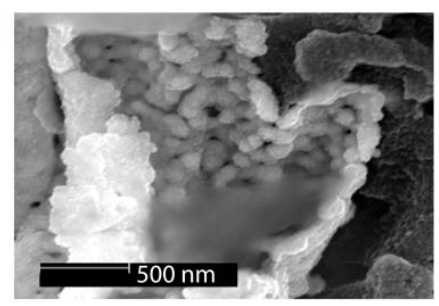

(C)

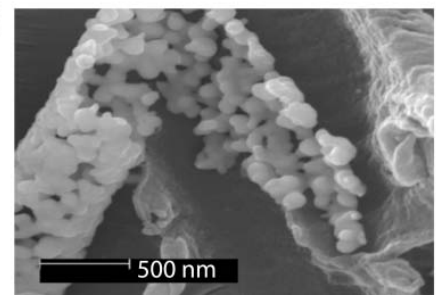

(B)

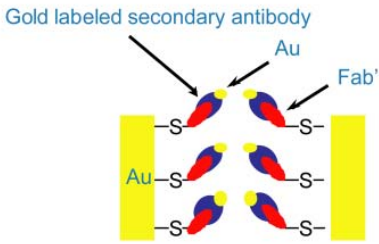

(D)

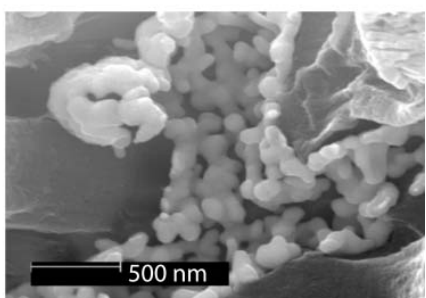

Figure 43. SEM micrograph of the inner pore surface of Au-lgG-Fab'-Au-NCAM and controls: (A) the surface of the pores with Au-IgG-Fab'-Au; (B) schematic representation of Au-lgG-Fab'Au-NCAM, the antibody-based molecular recognition gate for (A);

(C) cleaned Au-NCAM; and (D) control Au-NCAM.

Selective capture and release of human insulin was verified using matrixassisted laser desorption/ ionization time-of-flight mass spectrometry by examining the ability of suitably prepared Au-NCAM constructs to selectively capture and release a specific antigen, insulin, from a mixture also containing an acidic control peptide and a BSA blocker. Acidic peptide was selected as a control antigen because it produces a peak of similar intensity in the mass spectrum when analyzed from an equimolar mixture with insulin, thereby compensating for inherent differences in sensitivity among different analytes. Figure 44 (A) shows the spectrum of the standard mixture solution of insulin [(ii) $\mathrm{m} / \mathrm{z}$ 5809.1] and acidic peptide [(i) $\mathrm{m} / \mathrm{z}$ 2961.5], both at $5 \mu \mathrm{M}$. Next, a solution containing acidic peptide and insulin at the same concentration as the binding solution (500 $\mathrm{nM}$ of each analyte), but without BSA blocker, was prepared in the antigen releasing solution. The MALDI-TOF MS spectrum from a dried droplet of $1 \mu \mathrm{L}$ solution ( 0.5 pmole each on the sample spot) was acquired but did not show distinct $[\mathrm{M}+\mathrm{H}]^{+}$peaks of the analytes, viz. For Figure 44(B), the next onprobe releasate spectra were acquired by placing a piece of insulin-Fab'$\mathrm{Au}-\mathrm{NCAM}$ on the MALDI probe and then sequentially applying $1 \mu \mathrm{L}$ of release buffer and $1 \mu \mathrm{L}$ of MALDI matrix. Figure 44 (C), in contrast to Figure 44(B), depicts the on-probe releasate spectrum of insulin-Fab'-Au-NCAM and shows an intense $[\mathrm{M}+\mathrm{H}]{ }^{+}$peak of insulin at $\mathrm{m} / \mathrm{z} 5809.6$ [at (v)] along with the $[\mathrm{M}+2 \mathrm{H}]^{2+}$ peak of insulin at $\mathrm{m} / \mathrm{z} 2904.9$ [(iii)] and an unassigned peptide peak from BSA blocker at $\mathrm{m} / \mathrm{z}$ 3886.9. However, no peaks originating from acidic peptide were observed. Comparing the peak intensity of panel (B) and panel (C), it is clear that Fab'-Au-NCAM concentrates the 
analyte. To evaluate the performance of the selective capture and release ability of the antigen on Fab'-Au-NCAM, the relative intensity ratio of peak (v) to peak (iv), was calculated and averaged across multiple measurements ( 5 spectra for insulin-Fab'-Au- NCAM and 7 spectra for control AuNCAM). This measured a 23-fold increase in intensity compared to the control [ratio of (viii) to (vii); Figure 44 (D)].

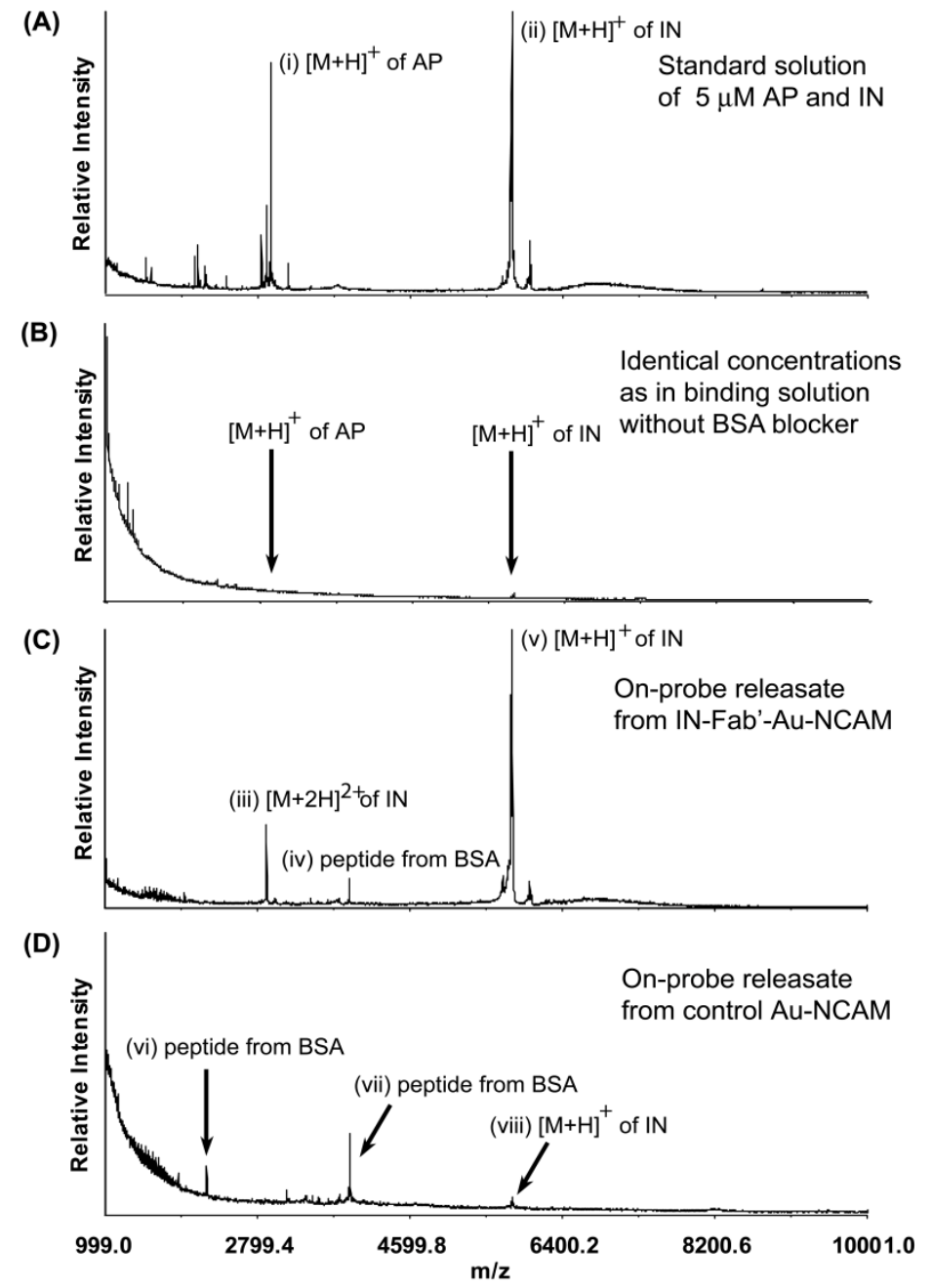

Figure 44. MALDI-TOF MS spectra of standard solution of $5 \mu \mathrm{M}$ Aplysia acidic peptide (control antigen: AP) and human insulin (antigen: IN), 5 pmole each on the probe (A); spectrum of 500

$\mathrm{nM}$ of AP and $500 \mathrm{nM}$ of IN solution prepared in release buffer without BSA blocker, 0.5 pmole each on the probe (B); on-probe release of captured peptide from Fab'-Au-NCAM (C); and on-probe release of captured peptide from control Au-NCAM (D). $m / z$ of peaks are (i) 2961.5, (ii) 5809.1, (iii) 2904.9, (iv) 3886.9, (v) 5809.6, (vi) 2912.3, (vii) 3886.1 and (viii) 5807.1. 


\subsubsection{Dual-function NCAMs}

This work pursued the incorporation of an Au nanocapillary array (NCAM) into a microfluidic device for dual purpose use, as both a microfluidic flow controller at cross channels and a highly sensitive plasmonic chemical sensor (see Figure 45). Enhanced subwavelength transmission has long been recognized for its potential as a sensing technique, and prior work in our group has demonstrated the use of a polymer NCAM as microfluidic flow controller. This project seeks to combine these two functions into dualmode metallic NCAM plasmonic sensors. Broadly divided, the objectives include:

(1) Optical characterization of the Au NCAM subwavelength transmission to identify spectral and spatial parameters important for chemical sensing.

(2) Fabrication of the freestanding array and integration into a 3-D microstructure, thereby exploring its capabilities as an electrokinetic flow controller.

(3) Plasmonic chemical sensing using the optical test bed to explore its detection capabilities under realistic analytical conditions.

(a)
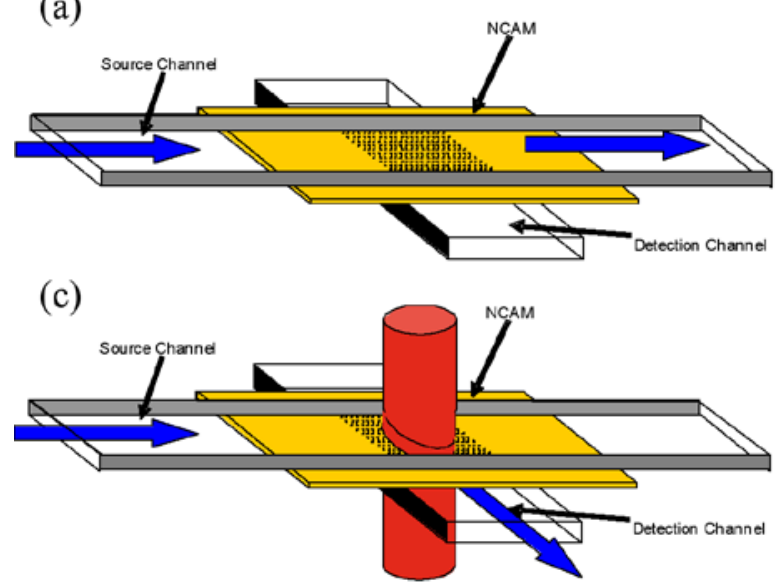

(b)

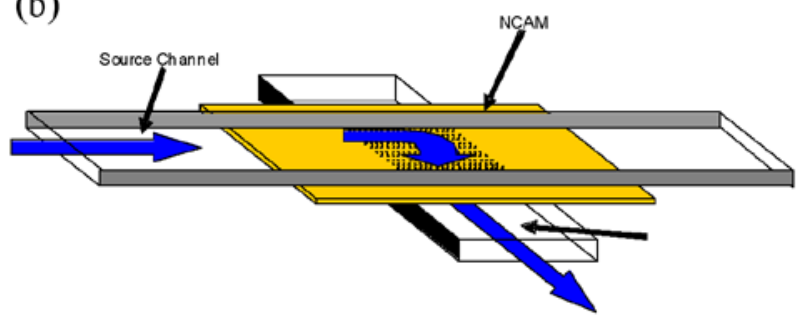

Figure 45. Multimodal concept for Au NCAM shown from top left, (a) and (b): microfluidic element in flow-past and flow-through configuration, respectively; (c) plasmonic chemical sensor.

Our work contributed to the first two objectives. The optical test bed depicted in Figure 46 allowed for spatial and spectral characterization of the subwavelength transmission. The tunable Ti:sapphire single band source enabled spectral information to be obtained at any wavelength and at 
much higher light levels than a white light source. Spatial information was obtained through the high numerical aperture condensing lens, measuring a wide range of $K$-space wave vectors in a single image. Additional work concerned the breakdown of incident angles into discrete experimental space and the combination of these experiments across the 700-1000 nm spectral range.

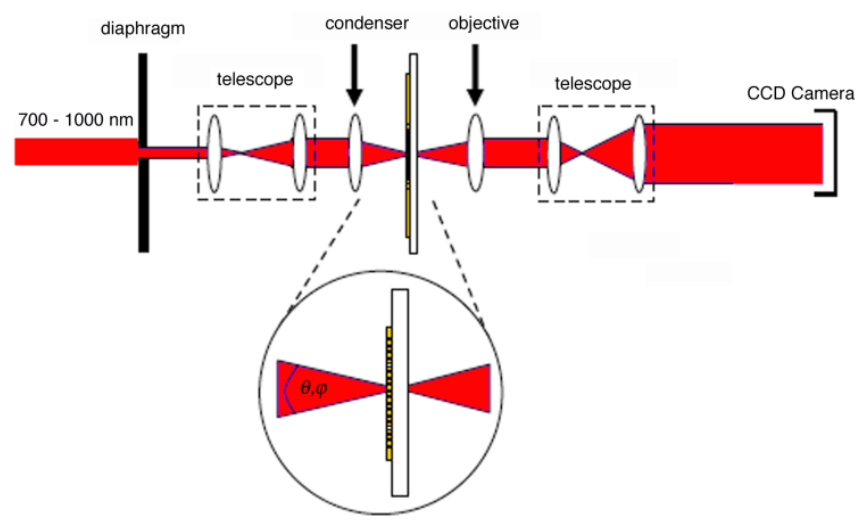

Figure 46. Optical test bed for characterization of subwavelength transmission. This setup enables characterization of both spatial $(\theta, \Phi)$ and spectral $(\lambda)$ information by tuning the Ti:sapphire single band source.

While working toward device fabrication, several Au NCAMs were prepared in a stepwise manner; the first step was thermal deposition onto a glass substrate followed by milling of the array at various aspect ratios using FIB (see Figure 47). These arrays were also used for optical characterization in the configuration shown in Figure 45. For the full 3-D structure, the NCAMs were milled on a thin polymer monolith rather than glass, and subsequently sandwiched between PDMS microchannels to create the 3-D structure. Ongoing experiments on deposition of Au onto thin polymer films showed good Au layer integrity down to a polymer thickness of 0.8 $\mu \mathrm{m}$.
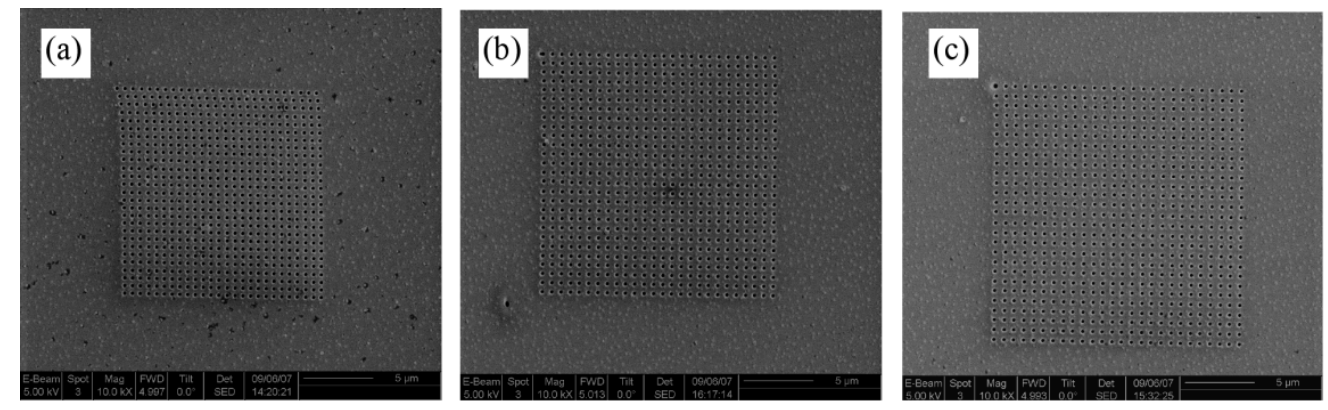

Figure 47. Au NCAMs milled in a $200 \mathrm{~nm}$ thick Au film on glass. In all cases, the pores are $230 \mathrm{~nm}$ in diameter and are milled in a $25 \times 25$ square array. Periodicity $a_{o}$ (distance centerto-center) is varied as follows: (a) $a_{o}=570 \mathrm{~nm}$, (b) $a_{o}=670 \mathrm{~nm}$, and (c) $a_{o}=710 \mathrm{~nm}$. 


\subsubsection{Floating injection in NCA-interconnected multilayer microfluidic} devices

We investigated fluidic manipulation techniques in NCA-interconnect multilayer microfluidic devices including a floating injection approach, which is capable of transporting reproducible sample plugs from one microfluidic channel to another through the NCA. This technique takes advantage of the electric field extension that takes place across the NCA when a voltage is applied across the injection channel or the separation channel while the other channel remain floated. A voltage is applied across the injection channel to electrokinetically pump analytes from the sample reservoir (S) to the sample waste reservoir (SW), c.f. (Figure 48). Analytes that are introduced through the NCA into the separation channel stay near the NCA region without electrokinetic expansion because the extension of the electrical field is reduced further away from the NCA. The current studies clarify the flow physics in NCA-interconnected microfluidics and describe strategies to achieve reproducible sample manipulation.

(a)
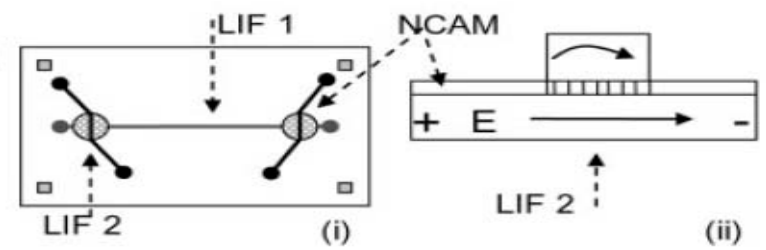

(b)
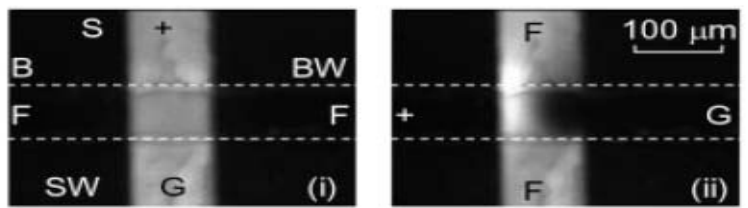

Figure 48. (a) Schematic diagram of the device (i) and the electric field extension across the NCA (ii); (b) images showing $\mathrm{FL}^{2-}$ - distribution during the floating injection and separation processes: (i) after floating injection from the sample (S/SW) microfluidic channel to the separation (B/BW) channel; and (ii) immediate application of separation potential (in the B/BW channel).

\subsubsection{Online sample concentration on NCA-interconnected, multilayer, microfluidic devices}

Selective ion transport in NCA (10 $\mathrm{nm}$ diameter) induces concentration polarization. We have demonstrated online sample concentration on a 3-D PMMA hybrid nano/ microfluidic device where two orthogonal electric fields, one across the NCA and the other across the microfluidic channel, induce concentration polarization as well as a concentrated analyte band. The concentration polarization caused by the electric field across the NCA 
is attributed to the extended Debye layer across the NCA. Figure 49 shows representative data acquired by (A) fluorescence microscopy, (B) laserinduced fluorescence (LIF) detection, and (C) analyte band concentration.
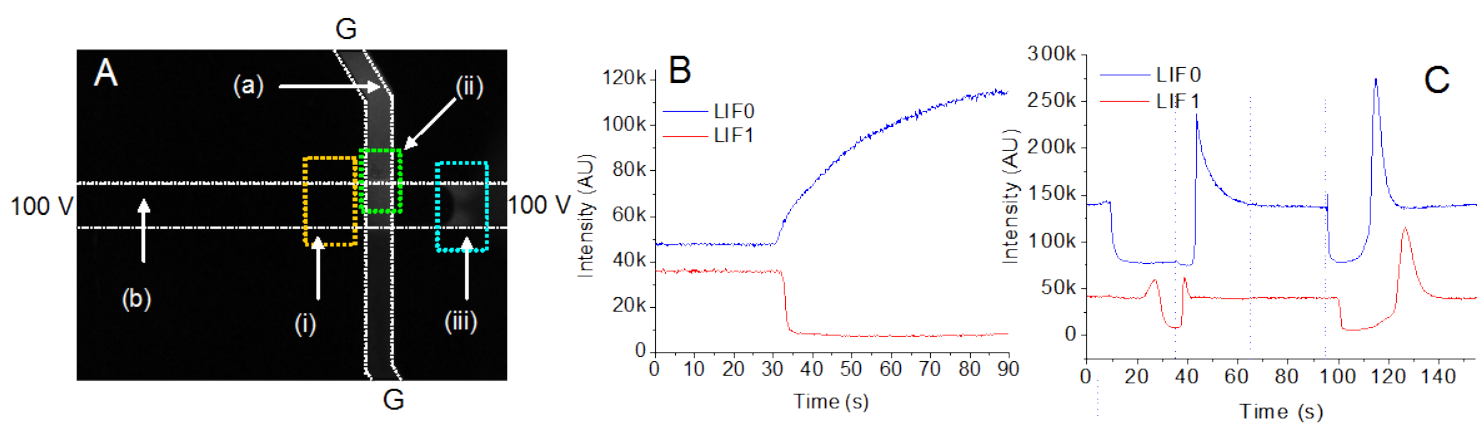

Figure 49. Concentration polarization and analyte band concentration demonstrated by using fluorescein ( $\left.\mathrm{FL}^{2-}\right)$ in $10 \mathrm{mM}$ phosphate buffer, $\mathrm{pH}$ 8.0. (A) is a fluorescence microscope image where (a) is a microfluidic channel above the NCA and (b) a microfluidic channel below the NCA; (i) shows ion depletion, (ii) shows ion accumulation and (iii) shows the focused analyte band. (B) and (C) are LIF data with different detection points. For (B), LIFO is focused at (ii) and LIF1 is focused at (i). For (C), LIFO and LIF1 are both focused along channel (b) to detect band movement (iii).

\subsubsection{Continuously coated NCAM}

NCAMs electrolessly coated over $1 \mathrm{~h}$ yield continuous gold films. Typically, after coating, pore size is reduced from $400 \mathrm{~nm}$ to between 200 and 250 $\mathrm{nm}$. Electrokinetic injection across such NCAMs was observed, but with very poor efficiency. Instead, the main effect of applied potential is manipulation of analyte concentration in the microfluidic channel. Probe molecules are depleted in the Au region and stacked near the edge of the Au region. The results of such a process are presented in a spatiotemporal fluorescence intensity plot in Figure 50. The spatial fluorescence intensity distributions in the source channel at given times are represented by slices parallel to the $x$ axis, and the time evolution of these slices is represented along the y axis. Alternatively, the temporal fluorescence behavior at a given point along the source channel is given by slices parallel to the y axis. Horizontal gridlines indicate a step in the applied potential sequence, with voltages indicated to the right of the spatiotemporal plot. The constant, low-intensity area between 180 and 360 pixels at all times is due to the low transmission of the gold coating at the intersection of microfluidic channels, as shown in the inset (upper/ middle right). The behavior of the system results from a complex interplay of all the driving forces present at any given time and can be interpreted starting from a few key assumptions: (1) Fluorescein exhibits a $\mathrm{pK}_{\mathrm{a} 2}=6.4$, so at the $\mathrm{pH}$ of the running 
buffer, it is negatively charged, and electrophoresis transports it toward the anode; (2) EOF is always from anode to cathode; (3) the left and right sides are asymmetric due to the consistent influence of pressure-driven flow from left to right; and (4) very little fluorescein is injected through the NCAM, so it must be accounted for within the source channel.
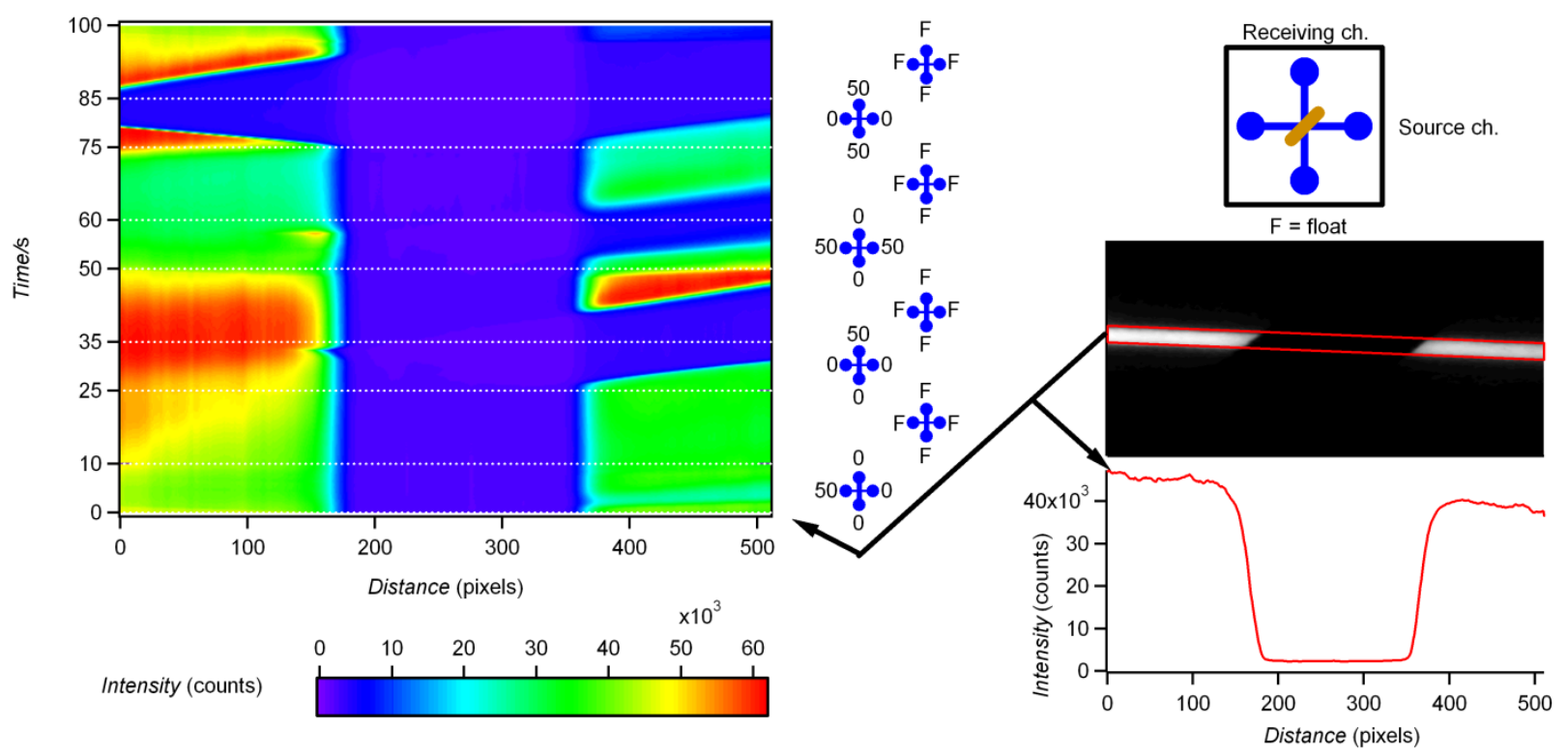

Figure 50. Fluorescein dynamics in a source channel. Attempted injection across $400 \mathrm{~nm}$ polycarbonate membrane coated with continuous gold film. There is no appreciable injection of fluorescein from the source channel into the receiving channel.

In order to understand these complex depletion and enrichment phenomena in more detail, fluid flow in the portion of the microchannel in contact with the Au-NCAM conductor was modeled via finite element simulations, the results of which are depicted in Figure 51. Panel (a) illustrates the potential distribution and velocity fields within the microchannel. Electric field strength in the vicinity of the gold segment is shown in panels (b) and (c) (magnified). The first thing to notice in Figure 51 is that the plug-flow velocity field is already being distorted near the edge of the Au-NCAM. The flow profile is semiparabolic above the Au patch due to the no-slip condition on the Au surface and the EOF condition at the top boundary. The potential applied along the channel causes a strong distortion of the electric field at the gold segment; its magnitude is reduced several-fold in comparison with the rest of the microchannel, and there is a significant increase in electric field strength at the very edge of the gold segment. This, in turn, affects the magnitude of electrophoresis in the vicinity of the gold edge. Specifically, an electrophoretic velocity component is introduced at the edge which opposes the electroosmotic/pressure-driven flow present in 
the rest of the channel. As a result, movement of charged particles is affected. In the case of fluorescein, EOF and electrophoretic flow are in opposite directions [indicated by a black arrow in Figure 51(c)], which is consistent with the inversion of the direction of transport that was observed in Figure 50. This leads to stacking of fluorescein at one edge of the $\mathrm{Au}-\mathrm{NCAM}$ and depletion at the other.
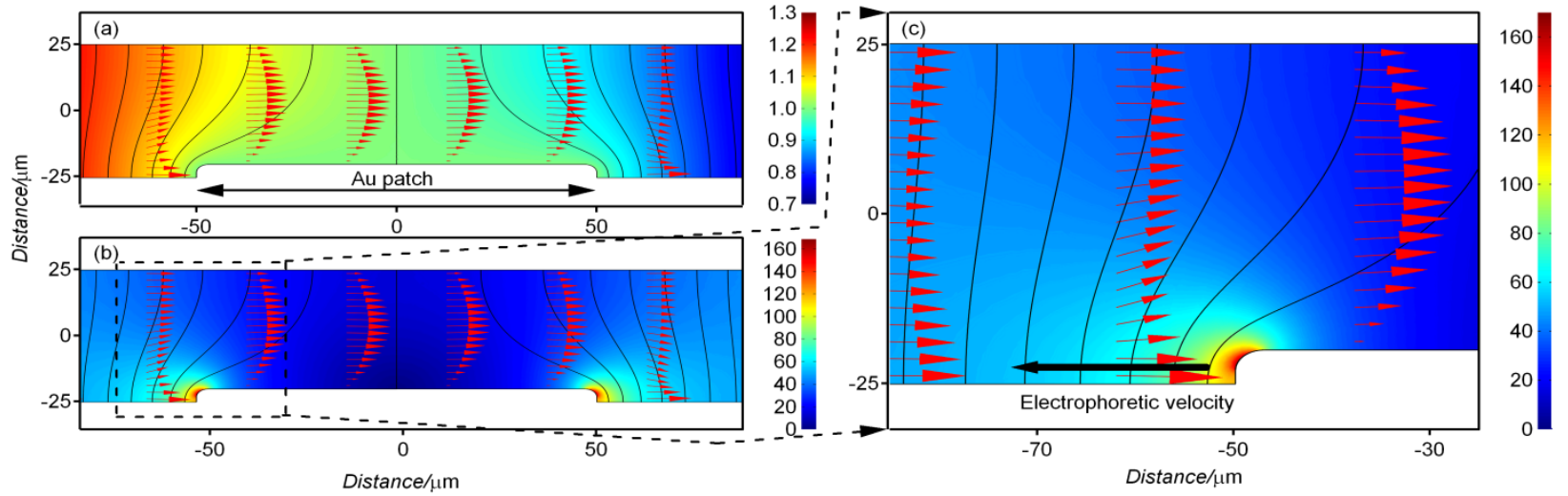

Figure 51. Finite element simulation of the electrokinetic flow near an Au segment in a polymer microchannel. In all three plots, red arrows depict the velocity field (electroosmotic), while black contour lines are lines of constant electrical potential. In (a), the color surface plot

depicts the electric potential; in (b), color depicts electric field strength. The peak field strength occurs just at the edges of the gold patch, where it reaches $160 \mathrm{~V} \mathrm{~cm}^{-1}$. (c) A magnified view of the left corner of the Au patch; the black arrow depicts the relative size of the electrophoretic velocity for fluorescein (electrophoretic mobility $=3.5 \mathrm{~cm}^{2} \mathrm{~V}^{-1} \mathrm{~s}^{-1}$ ) near the edge of the gold segment.

\subsubsection{One-side coated NCAMs}

Example of injection across NCAM coated with gold on the receiving side is shown in Figure 52. Analyte injection is EOF controlled; thus, injection occurs in the direction of a negatively biased channel. When bias magnitude is small, devices with NCAMs coated on one side perform similarly to uncoated polymeric NCAMs (see bottom trace, Figure 52), and an increase of bias potential leads to enhanced transport. However, continuous $\mathrm{Au}$ coating in the receiving channel limits the extent to which bias can be increased without adverse effects. At an intermediate bias magnitude ( $\sim 40-$ $60 \mathrm{~V}$ ), injection occurs only at transient stages (top trace in Figure 52). Further increase of bias leads to bubble formation in the receiving channel. The mechanism of injection across such NCAM is depicted in simulation results shown in Figure 53. Source microchannel and nanopore can support EOF due to nonzero surface charge. Gold coating at the exit of the nanopore establishes a zone with constant potential; thus, there is no elec- 
trokinetic driving force in the region. The fluid (and probe molecules) is propelled across the gold-coated region due to pressure-driven flow due to EOF pumping in the source microchannel and within the uncoated nanopores.

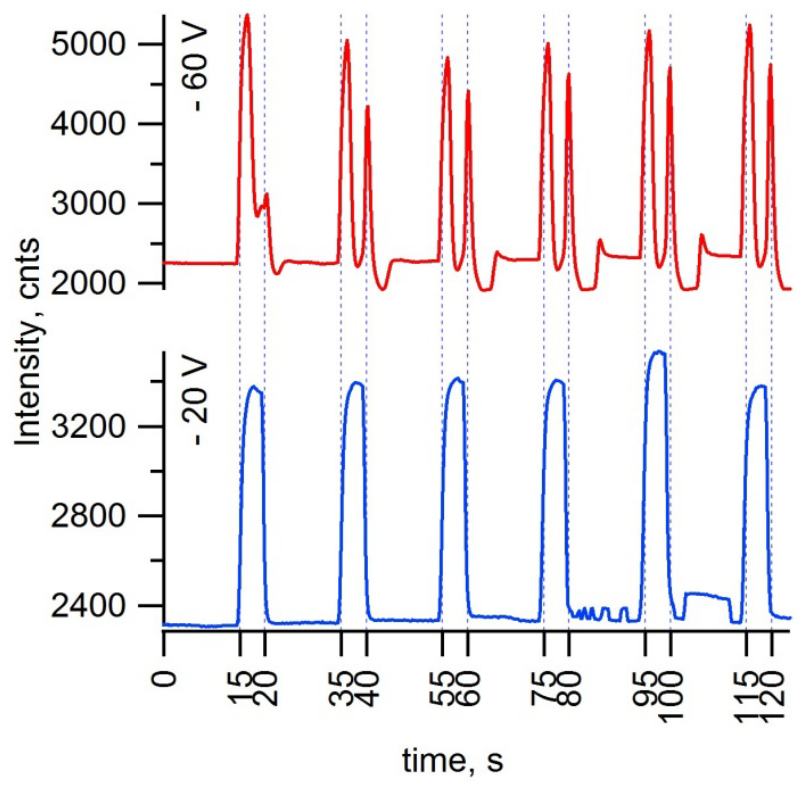

Figure 52. Injection across NCAM coated on one side. Each trace shows representative injections using intermediate (top) and small (bottom) bias.

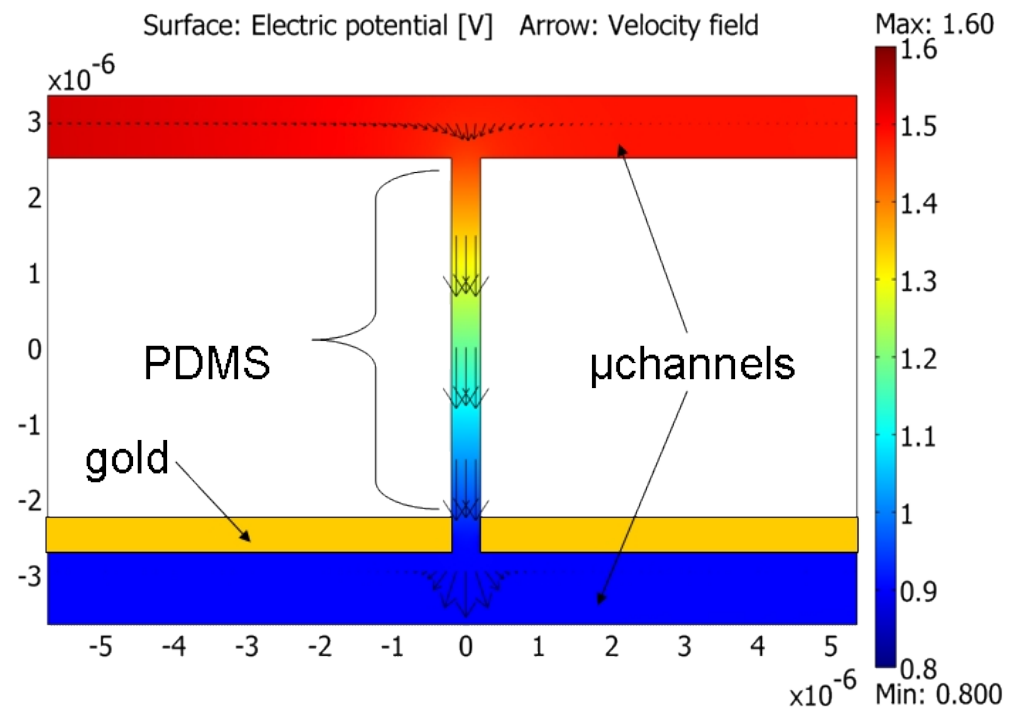

Figure 53. Simulation of EOF pumping across a single nanopore. Receiving side of NCAM is coated with Au. Color scale represents potential distribution within microchannel, and arrows represent flow field. 


\subsubsection{NCAMs with gold island film}

This type of film is the most similar to uncoated NCAMs. Similarly to onesided, gold-coated NCAMs, injection is dominated by EOF. An example of injection is shown in Figure 54. In contrast to previously shown film architectures, gold is present in electrically isolated, small islands. As a result, electrochemical control of the gold surface is not available in these films.

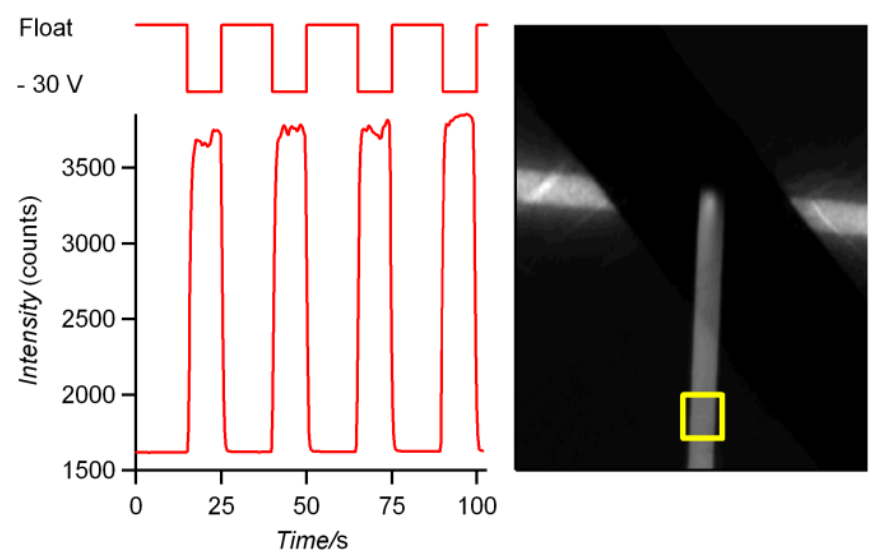

Figure 54. Fluorescence microscopy illustrating injection through an Au island-coated NCAM. Right: a single frame from an injection movie. The source channel is visible as the horizontal fluorescent area interrupted by a dark diagonal (Au-coated region). The receiving channel bias alternated between floating and $-30 \mathrm{~V}$ relative to the source channel to accomplish injection.

Left: a sequence of injections was followed by correlating the fluorescence intensity (left, bottom) integrated over the region of interest (yellow square), with the applied potential pulse train (left, top).

All of the above describes our efforts to study the effects of gold coatings on the nanochannels interconnects on bulk fluid flow. We have determined that minimizing the gold within the channel allows better control of fluid flow and analyte injections from a source microchannel to a receiving (detection) microchannel with minimal disruption in electroosmotic flow.

\subsection{Immobilization of DNAzyme on solid supports}

Much of the demonstrated research discussed above lends itself to the further advancement of revolutionary, miniaturized laboratories on a chip in terms of: device construction, understanding performance characteristics of microcomponents, chemistry within small volumes, and precise control of fluid flows. In order to develop a reliable and sensitive device for in situ measurement of heavy metals such as lead in ground water, we can proceed with attempts to incorporate the Pb-selective DNAzyme into a microfluidic-nanofluidic hybrid multilayer device containing a nanoscale fluidic 
molecular gate, consisting of a thin polycarbonate membrane with nanometer-sized perforations which can manipulate fluid flows and perform molecular separations on tiny volumes of material. $\mathrm{As}^{\mathrm{Pb}^{2+}}$ is transported to the DNAzyme, a fluorescent signal change can be detected upon cleavage of the substrate strand. This device is an important step toward detecting multiple heavy metal ions with a single injection by introducing different DNAzymes at different locations in the detection channel on a microfluidic-nanofluidic molecular gated device.

This section describes efforts and experiments to immobilize the DNAzyme to surfaces that would be present within microfluidic channels. Several methods have been developed for covalent attachment of modified oligonucleotides to pre-activated solid supports such as PMMA and glass. Chemical modification of PMMA substrates can be achieved by aminolysis and UV irradiation. Other modification methods include graft copolymerization, laser activation, ion bombardment, and plasma treatment.

There are several approaches to immobilizing DNAzyme in the microfluidic devices. As early proponents of this research, we reported on the preparation and use of gold coated polycarbonate NCAMs for the detection of $\mathrm{Pb}^{2+}$ in aqueous solutions using a ratiometric method (Wernette et al. 2006). The ratiometric method used an uncleavable substrate strand as a way to monitor the non-specific release of substrate away from the immobilized enzyme (Figure 55). It was observed that a slow kinetic and osmotically forced release of hybridized substrate caused an increase in detected fluorescence for cleavable substrate in the absence of $\mathrm{Pb}^{2+}$ analyte (a false positive). The contribution of this non-specific release was very dependent on the total amount of immobilized DNAzyme on the surface. The variable amount of total DNAzyme immobilized, as affected by differences in total surface area of the surface, was also a major source of variability in the final fluorescent intensities measured after $\mathrm{Pb}^{2+}$ reaction. Introduction of an uncleavable substrate strand with a different fluorophore provided a way to monitor the amount of non-specific release of substrate strand in the absence of $\mathrm{Pb}^{2+}$. Furthermore, the amount of non-specific release also standardized the variability in total amount of DNAzyme immobilized. 


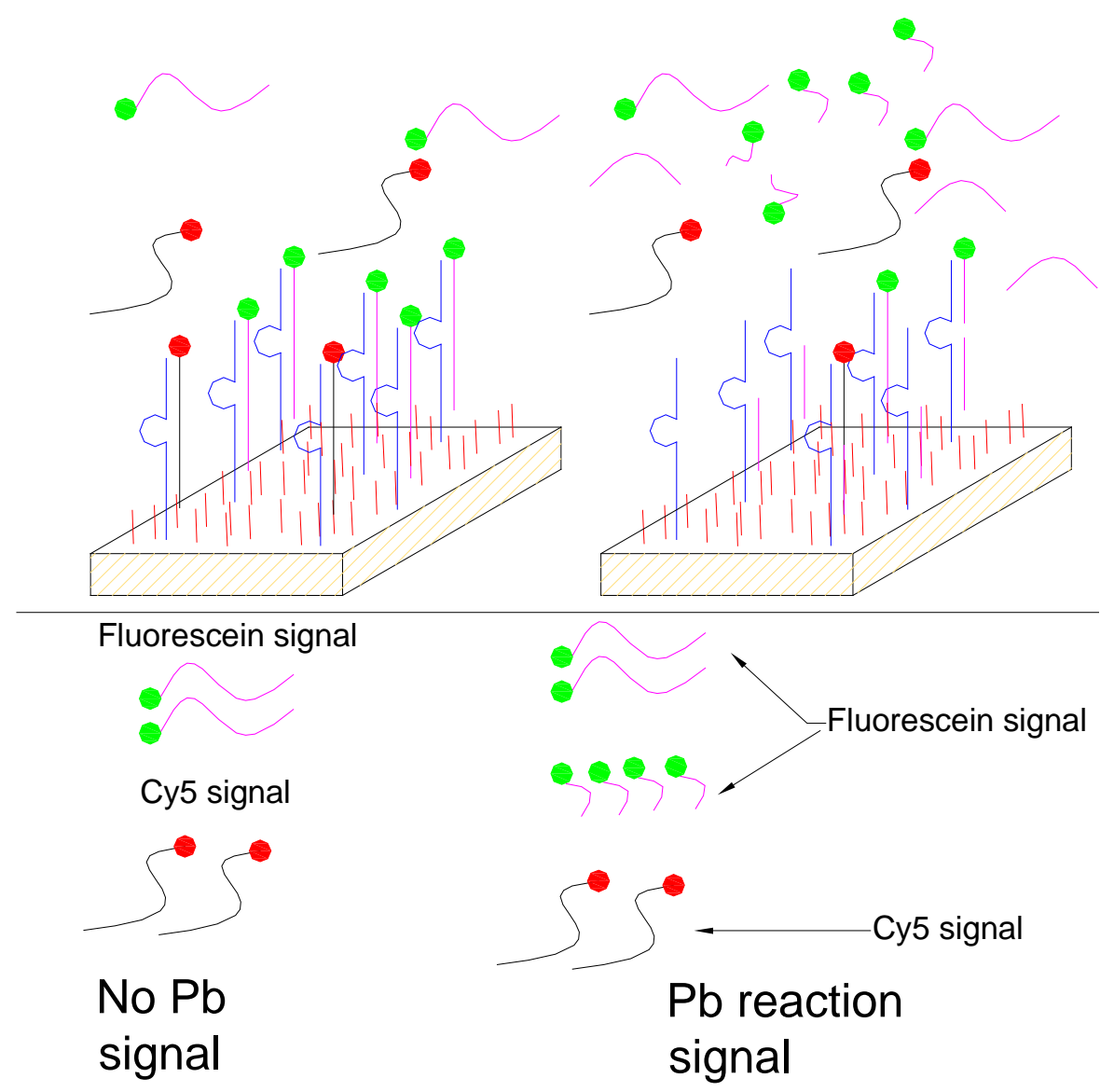

Figure 55. Schematic of the ratiometric internal control method before and after $\mathrm{Pb}^{2+}$ reaction to monitor non-specific release of substrate from immobilized enzyme. Cleavable substrate is shown as purple oligos with green heads, uncleavable as black oligos with red heads, and enzyme as blue oligos. Backfilling of the mercaptohexanol $(\mathrm{MCH})$ monolayer is represented by short red lines.

Previously, the ratiometric internal control method was proven effective at standardizing differences in cleaved intensity due to differences in total amount of DNAzyme because of surface area variances. This was shown by getting a standardized ratio for $10 \mu \mathrm{M} \mathrm{Pb}^{2+}$ reaction on NCAM tabs that were $2.5 \times 2.5 \mathrm{~mm}^{2}$ or $4.5 \times 5.0 \mathrm{~mm}^{2}$. Comparing $200 \mathrm{~nm}$ pore NCAM to $600 \mathrm{~nm}$ pore NCAM showed a difference in ratio and $\mathrm{Pb}^{2+}$ activity, despite the ratiometric method's ability to compensate for differences in surface area between the membranes (Figure 56). The $600 \mathrm{~nm}$ pore membrane showed $\sim 75 \%$ increase in activity as shown by comparing the red bars in the figure. 


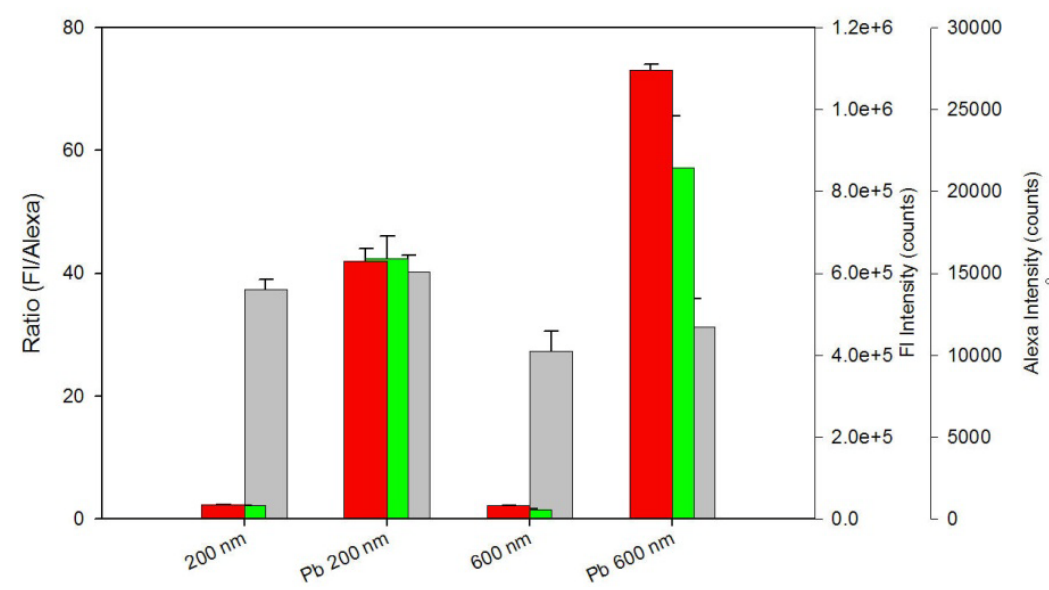

Figure 56. NCAM pore diameter effect on immobilized DNAzyme activity. $200 \mathrm{~nm}$ pore diameter NCAM has increased surface area compared to $600 \mathrm{~nm}$ due to higher pore density but has less activity. The decrease in activity is attributed to decreased diffusion and less active DNAzyme complex because of pore constraints. Uncleavable Alexa546 intensity is shown in gray; cleavable fluorescein in green; and the ratio of Fl/Alexa in red. Error bars represent one standard deviation of three independent reactions.

It was observed that the $200 \mathrm{~nm}$ pore NCAM indeed has more immobilized DNAzyme as indicated by an increase in uncleavable substrate signal (Alexa546 shown as the gray bars in Figure 56) which was used to monitor the total amount of DNAzyme complex.

The calculated increase in surface area of the $200 \mathrm{~nm}$ NCAM was 1.7 times that of $600 \mathrm{~nm}$, based on increase of pore density. The increase in uncleavable substrate signal was only 1.3 times that observed for $600 \mathrm{~nm}$. That slight decrease was attributed to decreased diffusion of enzyme and substrate through the $200 \mathrm{~nm}$ pores in the soak-based state used for immobilization and hybridization. Second, despite an observed increase in DNAzyme complex, there was less cleavable fragment fluorescence (fluorescein) observed, shown by the green bars in Figure 56. This must be attributed in part to a decrease of activity for the formed DNAzyme complex within the pores. We suspected that the pore wall constraints (i.e., the curvature of the pore walls forcing DNAzyme into closer proximity to one another) interrupt DNAzyme activity either by the DNAzyme interacting more with one another or with the pore walls. All experiments described hereafter used the 600-nm pore NCAM to ensure efficient diffusion through the pores and activity of the immobilized DNAzyme.

The ratiometric method gave a detection limit of $17 \mathrm{nM} \mathrm{Pb}^{2+}$ and demonstrated capacity for regeneration and repeated use. However, the problem with using gold-coated NCAMs is that due to the high background fluores- 
cence of the Au layer, the fluorescence measurements need to be done in solution, and this affects the reproducibility because some of the substrate strands are retained on the surface as a result of insufficient rinsing and mass transport constraints within the nanometer-sized pores.

In order to solve this problem, attempts were made to develop a sensor in which the $\mathrm{Pb}^{2+}$ can be quantified by the measurement of surface fluorescence using fluorescence microscopy, and by immobilization of fluorescently tagged DNAzymes on the surface of PMMA lining the walls of the microchannels (the detection channel of the microfluidic device). Upon hybridization, the fluorescence on the surface is quenched due to the proximity of the fluorophore to the quencher at the 3'-end of the substrate DNA. In the presence of $\mathrm{Pb}^{2+}$, the substrate strand is cleaved and released into solution, resulting in a measurable increase in the fluorescent signal on the surface. It has been shown in previous studies that surface immobilization does not affect the activity or specificity of DNAzyme. Current efforts focused on covalent attachment of fluorescein-tagged, modified oligonucleotides on pre-activated PMMA and glass.

\subsubsection{Surface modification of PMMA using aminated DNA}

In order to test the effectiveness of the chemical derivatization and hybridization processes, a random fluorescein (Fl)-tagged oligonucleotide strand [Am-PMMA(12)-Fl], modified with an amino (Am) group, was used that is not cleaved by $\mathrm{Pb}^{2+}$. The sequence is shown below.

$$
\text { 5'-NH } 2 \text {-C6-ATA-GTG-AGT-AGA-36 FAM-3' }
$$

The literature procedure reported by Soper and coworkers was used (Wang et al. 2003). Ethylene diamine (6 mmol) was placed in a roundbottomed flask and purged with nitrogen for $20 \mathrm{~min}$; $1 \mathrm{mmol} \mathrm{n}$-butyl lithium was added to the flask; and the mixture was stirred for $3 \mathrm{~h}$ in a purged, sealed vessel until a dark purple product was formed. PMMA slides were rinsed with isopropanol, followed by extensive rinsing with doubly deionized (dd) water and dried in a stream of nitrogen. The slide was purged with nitrogen in a sealed vessel for $20 \mathrm{~min}$ and then exposed to N-lithio ethylene diamine for $30 \mathrm{~min}$ after which the reaction was quenched with dd water and the PMMA slide was removed from the flask, rinsed with water, and dried under nitrogen. The aminated slides were then soaked in 5\% glutaric dialdehyde solution containing phosphate buffer ( $0.5 \mathrm{M}, \mathrm{pH} 6.4)$ for $2 \mathrm{~h}$. The 5'-amine terminated oligonucleotides $(20 \mu \mathrm{M}, 0.1 \mu \mathrm{L})$, sus- 
pended in $100 \mathrm{mM}$ phosphate buffer at $\mathrm{pH} 7$ were then spotted on the activated PMMA surface using a micropipette and the slides were left overnight in a humidified chamber at $25^{\circ} \mathrm{C}$. The oligonucleotides are covalently bonded on the surface through the formation of an imine bond as shown in Figure 57.

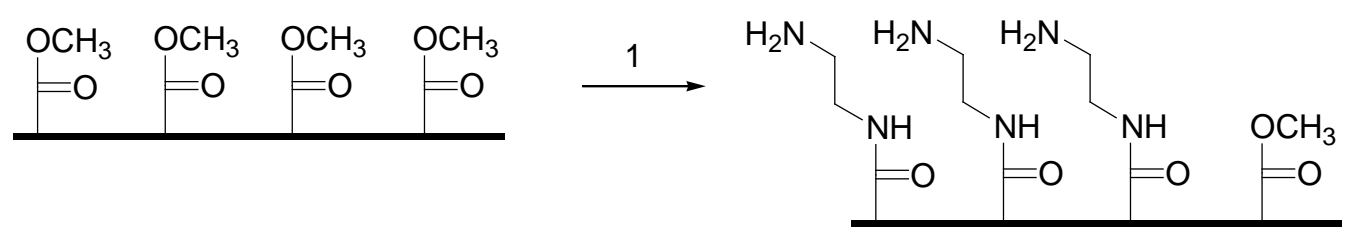

1. $0.13 \mathrm{~N}$-lithioethylenediamine in ethylenediamine, $30 \mathrm{~min}$
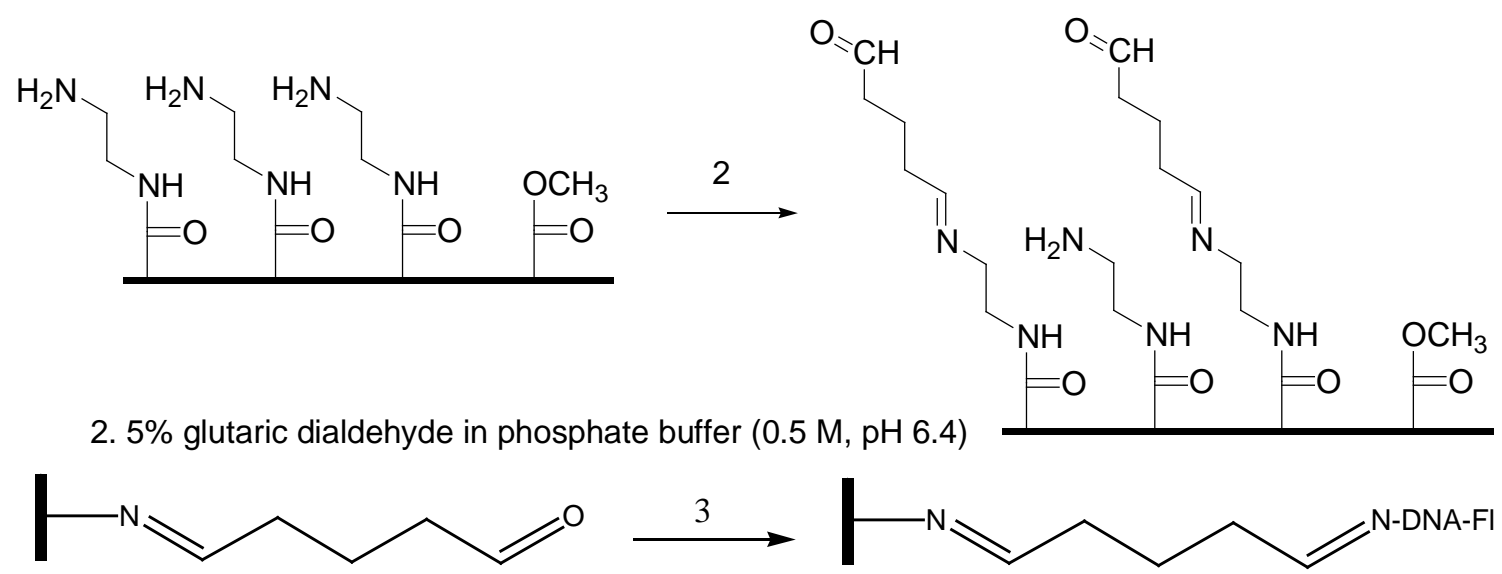

3. 20 uM DNA in $100 \mathrm{mM}$ phosphate buffer, pH 8.3, 12 h, r.t.

Figure 57. Immobilization of DNA on PMMA.

The PMMA slides were washed with $0.2 \%$ sodium dodecyl sulfate (SDS) at $25^{\circ} \mathrm{C}$ to remove unreacted DNA, followed by washing in water. The unreacted surface aldehyde groups were capped by reduction to alcohol using sodium borohydride $\left(\mathrm{NaBH}_{4}\right)$ in 10X phosphate-buffered saline (PBS) and ethanol. The slides were again washed with $0.2 \%$ SDS and water and dried in air.

In order to test for non-specific binding of DNA, two types of controls were used: (1) A fluorescein-tagged DNA strand without amine linker was spotted on chemically activated PMMA, and (2) Am-PMMA(12)-Fl was spotted on non-activated and cleaned PMMA slides.

The fluorescence of the immobilized DNA was detected by fluorescence microscopy with excitation at $488 \mathrm{~nm}$ using a 20X objective. 
The immobilized DNAzyme was hybridized with the complementary DNA (IB-PMMA(12)-Comp) in a hybridization solution containing $50 \mathrm{mMTris}$ buffer and $1 \mathrm{M} \mathrm{NaCl}$. The complementary DNA is attached to a fluorescence quencher, Iowa Black (IB). After spotting with complementary DNA, the slides were heated to $75^{\circ} \mathrm{C}$ in a water bath for $1 \mathrm{~h}$, cooled to room temperature for $1 \mathrm{~h}$, cooled to $4{ }^{\circ} \mathrm{C}$ for $30 \mathrm{~min}$, and warmed to room temperature for $15 \mathrm{~min}$ followed by washing, drying, and fluorescence measurement.

From results shown in Figure 58, it can be seen that a high packing density of DNA on PMMA is obtained by using Soper's method and results in high fluorescence intensity. Absence of fluorescence signals in the controls indicates no non-specific binding of DNA. From images D and E, the fluorescence signal seems to be substantially quenched upon hybridization, as compared to that of an unhybridized DNA spot taken at the same time. Image $\mathrm{E}$ is used as a control to check for photobleaching or self-quenching effects which can also cause a reduction in fluorescence intensity. 


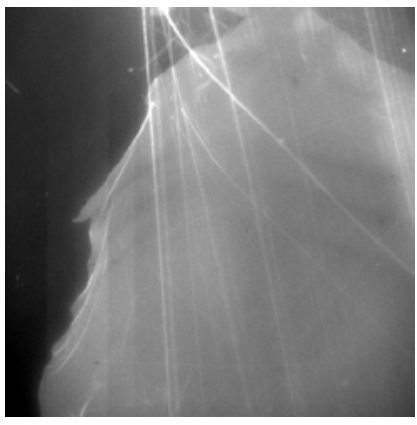

A

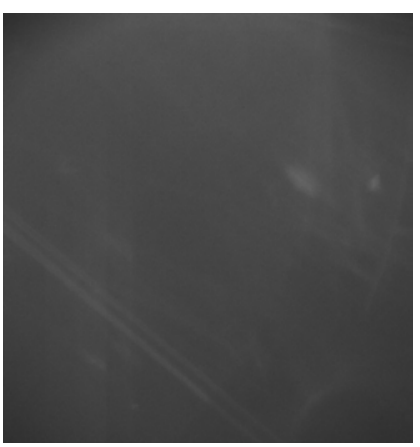

D

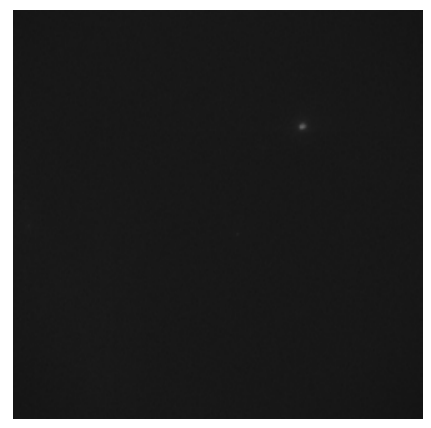

B

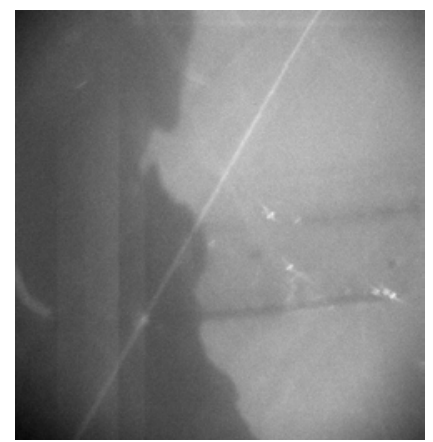

$E$

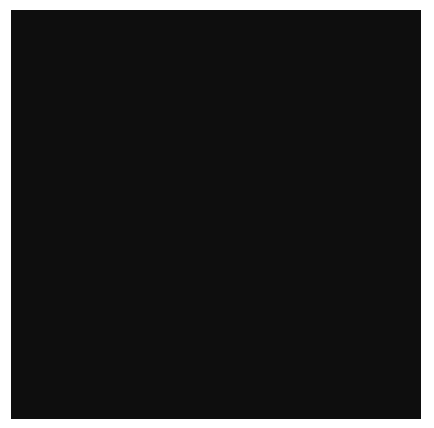

C

Figure 58. Immobilization of amine functionalized DNA [Am-PMMA(12)-FI] on PMMA substrate by Soper's method, showing fluorescence images: (A) DNA spotted on chemically activated PMMA; (B) control 1, non-aminated DNA on chemically activated PMMA; (C) control 2, AmPMMA(12)-FI on non-activated, cleaned PMMA substrate; (D) hybridized DNA on PMMA; and (E) image on unhybridized DNA spot taken at the same time as image D.

Immobilization of DNA was also carried out on UV-activated PMMA substrates to convert the surface ester groups to $\mathrm{COOH}$ according to Soper's method (Soper et al. 2005). The UV activation was carried out in a UV chamber for various times ranging from $5 \mathrm{~min}$ to $2 \mathrm{~h}$. The slides were then spotted with oligonucleotide solution $(100 \mu \mathrm{M}, 0.1 \mu \mathrm{L})$ in $0.1 \mathrm{M}$ MES at $\mathrm{pH}$ 5.5 containing $10 \mathrm{mM}$ of 1-ethyl-3-(3-dimethylaminopropyl) carbodiimide (EDC) and left overnight in a humidified chamber, followed by washing, drying, and fluorescence measurement. EDC catalyzes the formation of amide bonds between the surface $\mathrm{COOH}$ groups and aminated DNA. The procedure was repeated at $\mathrm{pH} 5.5$ and 4.5, but no DNA immobilization was observed. The slide exposed to UV for 1 or $2 \mathrm{~h}$ were partly frosted, indicating degradation of PMMA.

\subsubsection{Immobilization of DNAzyme on SuperAldehyde glass}

High-density amino and fluorophore modified DNAzymes were printed onto SuperAldehyde ${ }^{\circledR}$ glass slides (Telechem International, Inc., 
Sunnyvale, CA) using a Gene Machines OmniGrid $100{ }^{\circledR}$ Microarrayer. Since the slides were already covered with surface aldehyde groups, the amino terminated DNAzyme readily bonded to this surface. The slide was allowed to dry for $12 \mathrm{~h}$ at room temperature $\left(\sim 25^{\circ} \mathrm{C}\right)$ at $<30 \%$ relative humidity. The printed slides were then rinsed with $2 \times 0.1 \%$ SDS and then $2 \mathrm{x}$ dd $\mathrm{H}_{2} \mathrm{O}$ for 2 min each at room temperature to remove unbound DNA, followed by $5 \mathrm{~min}$ of gentle mixing in $500 \mathrm{~mL} \mathrm{NaBH}_{4}$ reducing solution to block the unreacted surface. After the blocking treatment, the slides were rinsed with $2 \mathrm{x}$ dd $\mathrm{H} 2 \mathrm{O}, 1 \times 0.1 \%$ SDS, then transferred to boiling dd $\mathrm{H}_{2} \mathrm{O}$ at $100{ }^{\circ} \mathrm{C}$ for $3 \mathrm{~min}$, followed by plunging the slides into ice cold $100 \%$ ethanol for $30 \mathrm{sec}$ to fix the denatured DNA. A $1 \mu \mathrm{M}$ Cy3 labeled substrate DNA was spotted on top of the immobilized DNAzyme. The glass slide was then taken to a hybridization chamber that sat on a water bath (temperature set to $95^{\circ} \mathrm{C}$ ) for $15 \mathrm{~min}$, and then slowly cooled to room temperature. The hybridization time was $24 \mathrm{~h}$.

After completion of hybridization, the slide was quickly transferred to a beaker containing Wash Buffer A ( 1 x SSPE + 0.2\% SDS). Following a 5min incubation in Wash Buffer A, the slide was transferred to a second beaker containing Wash Buffer B (0.1x SSPE + 0.2\% SDS). Wash Buffer B works well to remove the remaining unhybridized material. After a $5 \mathrm{~min}$ incubation in Wash Buffer B, the microarray slide was transferred to Wash Buffer C consisting of 0.1x SSC for 30 sec at $25^{\circ} \mathrm{C}$ to remove trace SDS. The microarray slide was dried and scanned.

Figure 59 shows the microarray results for immobilization of DNAzyme onto SuperAldehyde glass. The red spots indicate the presence of immobilized DNAzyme due to the Cy5 label. All amine terminated DNAzymes bind well to the SuperAldehyde glass. Cy3-labeled substrate DNA that hybridizes to the DNAzyme will turn the resultant spot yellow due to mixing of emission from Cy5 and Cy3. The results indicated there was complete hybridization between the DNAzyme and the substrate DNA only in the first row. The hybridization efficiency was clearly influenced by the position of the fluorophore. The substrate DNA can hybridize to row 1 DNAzyme since the Cy3 group extends out into solution. Steric hindrance from the bulky Cy3 group at 3' end makes it difficult to insert into all other DNAzyme strands to complete hybridization since the Cy3 group is oriented toward the slide. Therefore, row 2 to 4 only showed partial hybridization (from the ratio of the fluorescence intensity of Cy3 to Cy5). Negligible Cy3 signal from reference row 5 indicated that non-specific interactions 
between the substrate DNA and the SuperAldehyde glass slide do not occur.

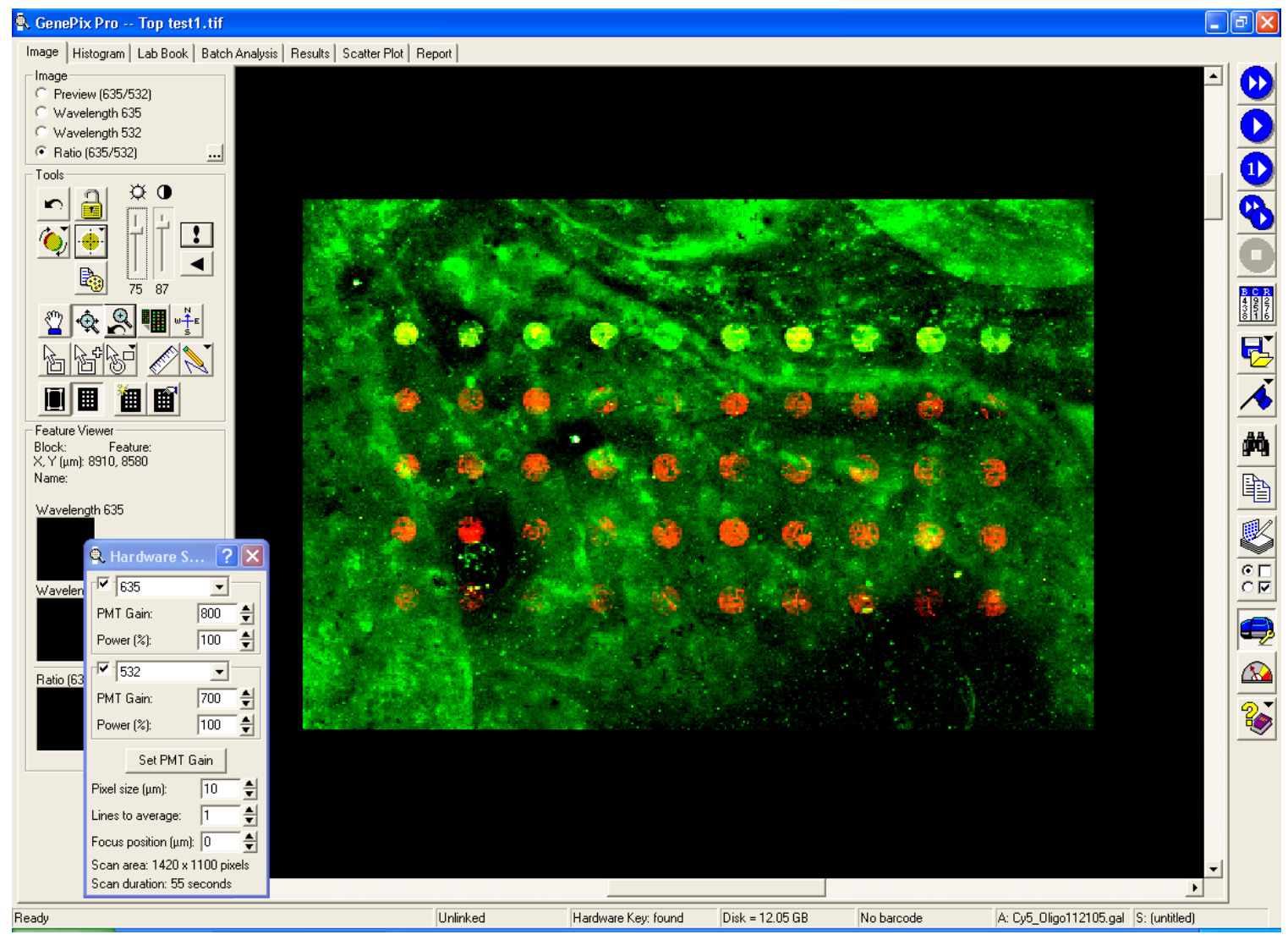

Figure 59. Microarray image of 50 spots of DNAzymes attached to the SuperAldehyde glass. Each row is a different DNA sequence immobilized as detailed below (from the top):

Row 1: 5'-Cy5- TTा TTA AAG AGA CAT CTC TTC TCC GAG CCG GTC GAA ATA GTG AGT$\mathrm{NH}_{2}-3^{\prime}$

Row 2: $\quad$ 5'-NH 2 -T TIT TCA TCT CTT CTC CGA GCC GGT CGA AAT AGT GAG T-Cy5-3'

Row 3: $\quad$ 5'-NH 2 -TTा TाT TाT TCA TCT CTT CTC CGA GCC GGT CGA AAT AGT GAG T-Cy5-3'

Row 4: 5 '-NH Cy5-3'

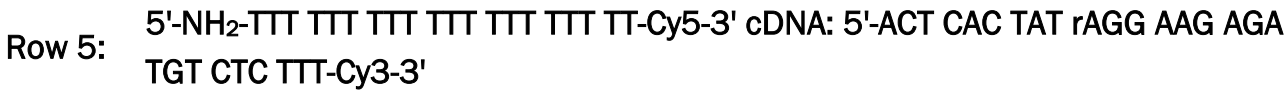

The oligonucleotide [Am-PMMA(12)-Fl] suspended in immobilization solution was spotted on SuperAldehyde glass slides (Telechem International, 
Inc., Sunnyvale, CA) using a micropipette in a similar fashion as previously described. The slides were then air dried for $1 \mathrm{~h}$ and fluorescence was measured at $488 \mathrm{~nm}$.

Although difficult to see, the fluorescence images in Figure 60 show immobilization of fluorescently tagged DNA on the surface. Absence of fluorescence signals in the control indicates no non-specific binding of DNA. However, the fluorescence signal intensity is very weak, which may be caused by low DNA surface coverage or high background fluorescence due to incomplete blocking of surface aldehyde groups. The spots have more well-defined edges, probably due to the more uniform coverage of surface aldehyde groups on the SuperAldehyde glass compared to that achieved on PMMA by Soper's method. The quality of spotting seems to be slightly improved in the commercial immobilization buffer compared to 3X SSC. However, there is a decrease in fluorescence intensity at the higher concentration of $100 \mu \mathrm{M}$ which may be due to self-quenching of closely spaced DNA strands at higher concentration.

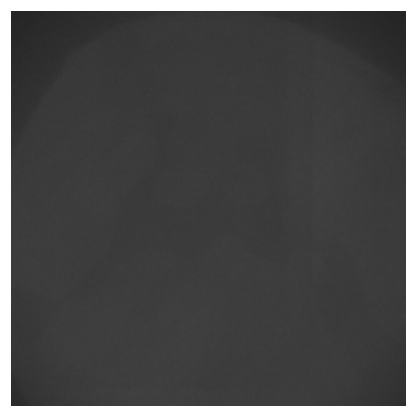

A

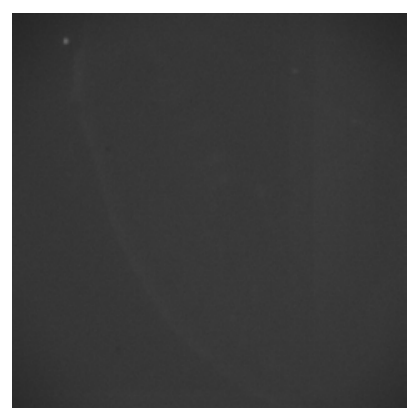

D

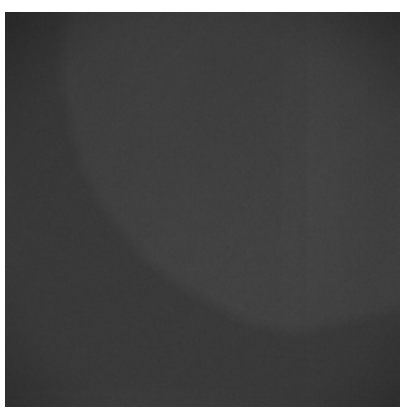

B

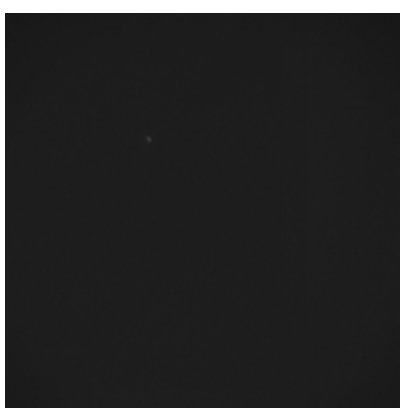

$\mathrm{E}$

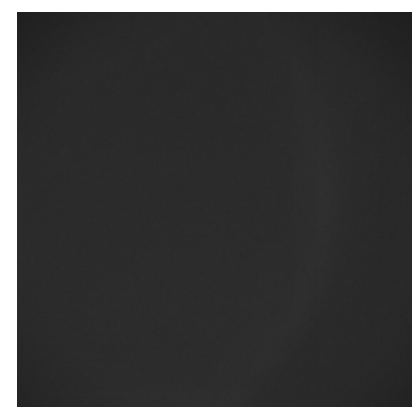

C

Figure 60. Immobilization of amine functionalized DNA [Am-PMMA(12)-FI] on SuperAldehyde glass: (A) $20 \mu \mathrm{M}$ DNA in 3X SSC; (B) $20 \mu \mathrm{M}$ DNA in 1X Microspotting Solution Plus; (C) $100 \mu \mathrm{M}$ DNA in 3X SSC; (D) $100 \mu \mathrm{M}$ DNA in 1X Microspotting Solution Plus; and (E) control-spot of non-aminated DNA. 


\subsubsection{Immobilization of DNAzyme on superstreptavidin glass}

Immobilization of DNAzyme was also carried out on superstreptavidin glass slides (Telechem International, Inc., Sunnyvale, CA). This consists of Superclean glass surfaces activated with streptavidin to bind biotinylated biomolecules. Streptavidin is protein isolated from Streptomycetes avidinii that has an irreversible binding affinity for biotin and is covalently bonded to the surface. The surface should have a low intrinsic fluorescence background and high binding capacity with each streptavidin molecule binding to four biotin molecules.

The biotinylated DNAzyme (Bio-17E-Fl) $(20 \mu \mathrm{M})$ labeled with fluorescein at the 5'-end was suspended in 1X Microspotting solution Plus (Telechem International, Inc., Sunnyvale, CA) and spotted on the superstreptavidin substrates. The substrates were then left overnight in a chamber at room temperature at $<30 \%$ relative humidity and then blocked with superstreptavidin blocking buffer $(40 \mu \mathrm{L})$. The blocking reaction was done under a coverslip for $1 \mathrm{~h}$ and helps in the reduction of background fluorescence. The slide was then washed for 5 min each with $1 \mathrm{X}$ Wash Buffer A and $1 \mathrm{X}$ Wash Buffer B, then dunked for 1s in $1 \mathrm{X}$ Wash Buffer C, followed by rinsing with dd water and drying in air for $1 \mathrm{~h}$. Wash Buffers $\mathrm{A}, \mathrm{B}$, and $\mathrm{C}$ have progressively lower salt content and help to remove physisorbed DNA on the surface. Wash Buffer $C$ is used to removed salts present in Wash Buffer B to avoid formation of streaks on the surface. The immobilized DNAzyme was hybridized with the complementary DNA [IB-(4) $17 \mathrm{~S}$ (7)] in a hybridization solution containing $50 \mathrm{mM}$ Tris buffer and $1 \mathrm{M}$ $\mathrm{NaCl}$. The complementary DNA is attached to a fluorescence quencher, Iowa Black. After spotting with complementary DNA, the slides were heated to $75^{\circ} \mathrm{C}$ in a water bath for $1 \mathrm{~h}$, cooled to room temperature for $1 \mathrm{~h}$, cooled to $4{ }^{\circ} \mathrm{C}$ for $30 \mathrm{~min}$, and warmed to room temperature for $15 \mathrm{~min}$ followed by washing, drying, and fluorescence measurement.

For the $\mathrm{Pb}^{2+}$ reaction, the substrate was soaked in $50 \mathrm{mM}$ Tris acetate buffer (pH 7.2) +50 mM NaCl for 5 min to remove physisorbed or unhybridized substrate DNA and then soaked for $1 \mathrm{~h}$ in $10 \mu \mathrm{M} \mathrm{Pb}^{2+}$ acetate - $3 \mathrm{H}_{2} \mathrm{O}$ in $50 \mathrm{mM}$ Tris acetate buffer $(\mathrm{pH} 7.2)+50 \mathrm{mM} \mathrm{NaCl}$. After the reaction with $\mathrm{Pb}^{2+}$, the substrate was washed again with $50 \mathrm{mM}$ Tris acetate buffer (pH 7.2) +50 mM NaCl for $5 \mathrm{~min}$, rinsed with water, and dried. The fluorescence was measured at $488 \mathrm{~nm}$. 
In Figure 61, the fluorescence image A shows a high surface coverage of biotinylated DNAzyme on the superstreptavidin glass substrate, resulting in high fluorescence intensity compared to the control spot. The fluorescence signal is also more uniform on this substrate. The control spot gives a very weak fluorescence signal, indicating some amount of non-specific binding. From image $C$, the fluorescence signal seems to be completely quenched on hybridization as expected. Upon reaction with $\mathrm{Pb}^{2+}$, the quencher-labeled substrate DNA is cleaved and released, resulting in an increase in fluorescence signal intensity.

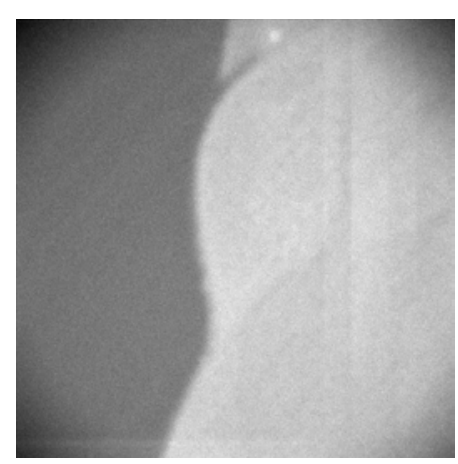

A

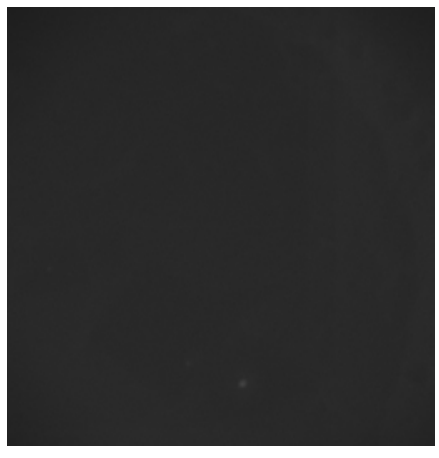

C

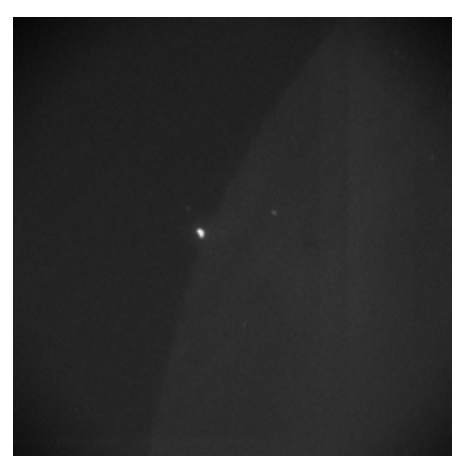

B

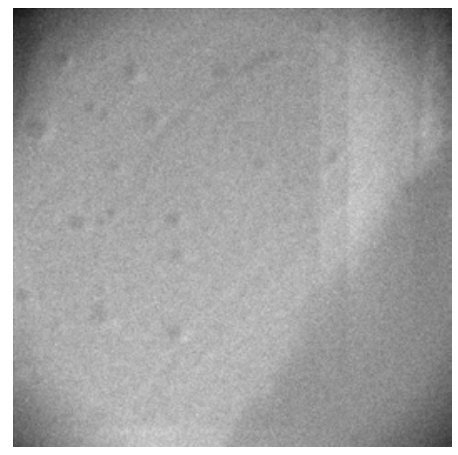

D

Figure 61. Immobilization of fluorescein-tagged, biotin modified DNAzyme (Bio-17E-FI) on superstreptavidin glass (SSG) substrate: (A) DNAzyme on SSG; (B) control for non-specific binding using Am-PMMA(12)-FI on SSG; (C) surface after hybridization; and (D) surface after reaction with $\mathrm{Pb}^{2+}$.

\subsubsection{Testing the addition of TCEP}

An experiment was conducted to investigate the importance of addition of tris (2-carboxyethyl) phosphine (TCEP) during self-assembly of the enzyme strand onto gold. TCEP was used to assist in breaking disulfide linkages in the modified DNA. However, the thiol-gold bond strength was higher than that of a disulfide bond; therefore, disulfide linkages should 
break while the gold-thiol bond should remain unaffected. To test this, immobilization solutions containing $100 \mu \mathrm{M}$ freshly prepared TCEP, 100 $\mu \mathrm{M}$ "stale" TCEP from a months-old stock solution, and no TCEP were used during self-assembly of the enzyme (Figure 62). The results showed TCEP makes no difference in total immobilization of the DNAzyme as indicated by all non-cleavable intensities being equal within error. However, the addition of "stale" TCEP drastically reduced the activity of the DNAzyme by nearly $40 \%$, compared to both fresh TCEP and TCEP-absent samples. We were uncertain how the stale TCEP could be affecting the DNAzyme because the total immobilized amount was the same and several rinsing steps were implemented after immobilization. Furthermore, although TCEP is necessary for reduction of disulfide bonds for efficient selfassembly of DNA onto gold nanoparticles, TCEP was not needed here. This likely was due to stabilizing molecules on gold nanoparticles which prevented the approach of DNA to the gold surface. TCEP was required to break the stabilizing molecular bonds to allow penetration of the thiol through the stabilizing layer and onto the gold. Such stabilizing molecules were not present on the planar or Au-NCAM surfaces.

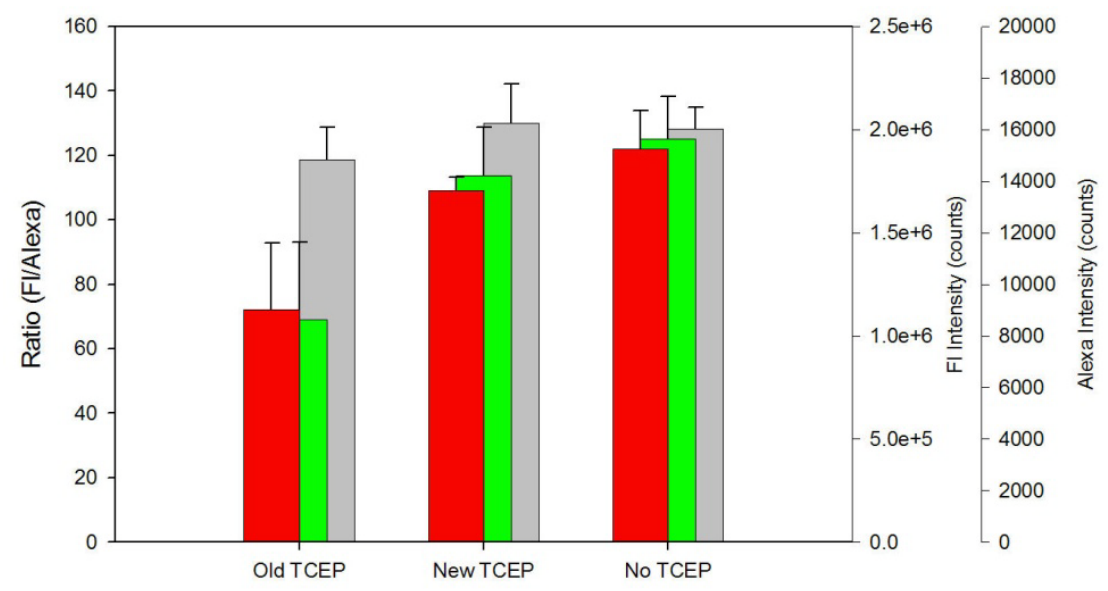

Figure 62. The effect of TCEP used for breaking disulfide linkages on immobilization of DNAzyme enzyme strand. TCEP has no effect on total DNAzyme immobilization as evidenced by the equal amounts of uncleavable standardization strand for all cases. Months-old TCEP does diminish the activity of the DNAzyme relative to fresh and TCEP absent samples but the effect on the oligo is not understood. The TCEP must directly alter the enzyme strand since numerous rinsing steps before $\mathrm{Pb}^{2+}$ reaction should completely remove TCEP from the NCAM DNAzyme surface. Error bars represent one standard deviation of three independent reactions. 


\subsubsection{Role of $\mathrm{MCH}$ backfilling layer in DNAzyme activity}

It was questioned whether or not the mercaptohexanol (MCH) backfilling layer was necessary for activity, a step that adds considerable challenges if immobilization is to be attempted within an assembled microfluidic device. Tarlov and co-workers (Herne and Tarlov 1997; Steel, Herne, and Tarlov 1998) used MCH to displace non-specific binding of DNA bases to the Au surface while simultaneously ensuring the DNA portion of the monolayer is not so dense as to cause steric or osmotic resistance for complimentary oligomers. The net effect was an overall increase in hybridization efficiency. The NCAMs were assembled with and without $\mathrm{MCH}$ to compare the activity of DNAzyme on NCAMs as dependent on the MCH monolayer (Figure 63). It was shown that the $\mathrm{MCH}$ provided a 3.5-fold increase in activity for the immobilized DNAzyme.

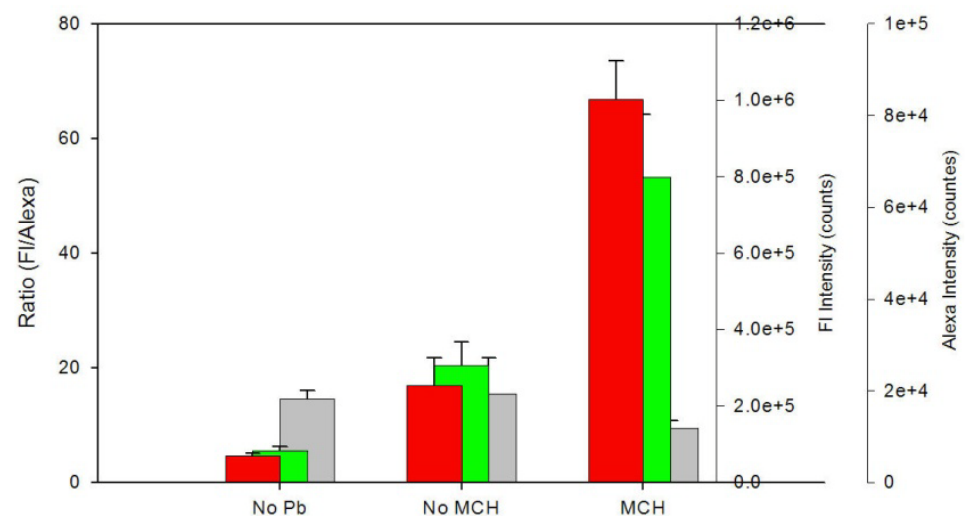

Figure 63. Activity of DNAzyme on NCAM, in the absence and presence of a backfilling MCH monolayer. The $\mathrm{MCH}$ monolayer causes a $\sim 3.5$ fold increase in activity by creating more active DNAzyme complexes. Uncleavable Alexa 546 intensity is shown in gray, cleavable fluorescein in green, and the ratio of Fl/Alexa in red. Error bars represent one standard deviation of four independent reactions.

The uncleavable substrate signal was higher in the $\mathrm{MCH}$ absent system than in the $\mathrm{MCH}$ present system. It has been shown that non-specifically bound oligomers are strongly held by the interacting surface, indicating the increased release of uncleavable substrate must be due to an increase in the total hybridized substrate and not from an increase in the amount of physisorbed substrate. This is likely due to $\mathrm{MCH}$ displacing a small portion of both specifically and non-specifically bound enzyme. However, while more substrate was shown to be hybridized, it was thought to be incompletely hybridized, thus decreasing the amount of fully assembled complex. Furthermore, the lower activity in the $\mathrm{MCH}$ absent case could be due to the assembled DNAzyme complex interacting with the gold surface, 
rendering it inactive by preventing formation of the active tertiary structure thought necessary for cleavage. In either case, the ratiometric system showed that the $\mathrm{MCH}$ monolayer is necessary for optimal formation of active DNAzyme complex on the gold surface.

\subsubsection{Effect of $\mathrm{NaCl}$ concentration on DNAzyme activity}

Another important factor for DNAzyme activity was $\mathrm{NaCl}$ concentration, due to its effect on complex stability and release of cleaved fragment. As reported previously, the enzyme/ substrate arms were optimized for a 50 $\mathrm{mM} \mathrm{NaCl}$ reaction solution during solution activity experiments. Here, the effect of reaction solution $\mathrm{NaCl}$ concentration on immobilized DNAzyme activity was studied by performing the $\mathrm{Pb}^{2+}$ reaction in various $\mathrm{NaCl}$ concentrations after rinsing in the corresponding $\mathrm{NaCl}$ solution for $5 \mathrm{~min}$. $\mathrm{NaCl}$ concentrations were varied from $\mathrm{NaCl}$ absent to $1 \mathrm{M} \mathrm{NaCl}$ as shown in Figure 64. The results showed the system was still optimized for $50 \mathrm{mM}$ $\mathrm{NaCl}$ and that immobilization of the DNAzyme complex had not altered the optimal $\mathrm{NaCl}$ concentration. This relatively low $\mathrm{NaCl}$ concentration should provide a suitable ionic strength for electroosmotic flow and gated injection within the microfluidic device.

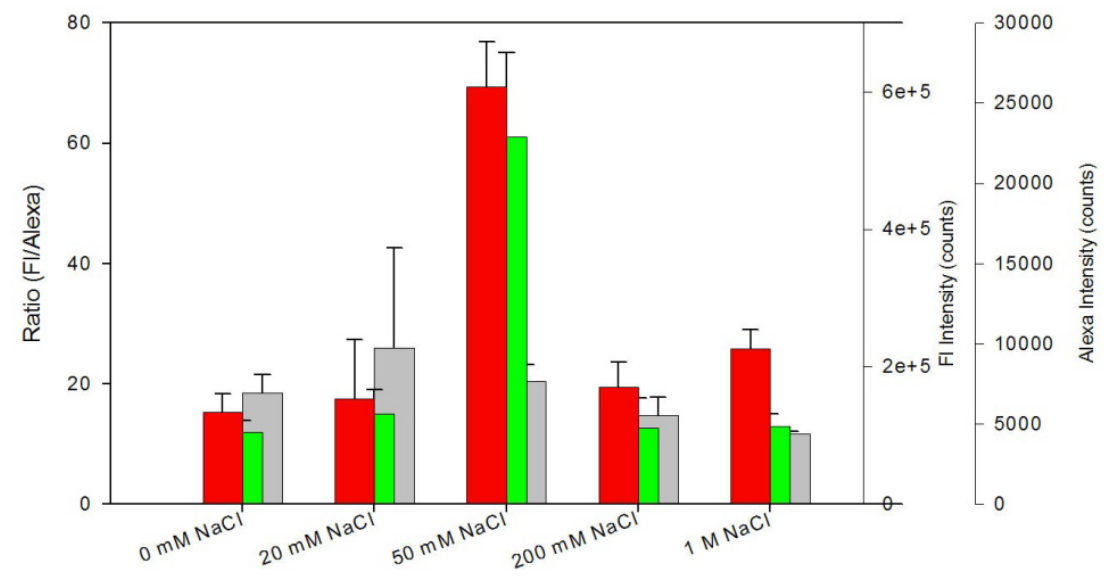

Figure 64. $\mathrm{NaCl}$ effect on DNAzyme activity at various concentrations in reaction solution. System is shown to be optimized at $50 \mathrm{mM} \mathrm{NaCl}$, with lower concentrations resulting in unstable complex and higher concentrations inhibiting cleaved fragment release. Uncleavable Alexa 546 intensity is shown in gray, cleavable fluorescein in green, and the ratio of Fl/Alexa in red. Error bars represent one standard deviation of three independent reactions.

The ratiometric system allowed for additional analysis to be made because all DNAzyme NCAMs were hybridized in $1 \mathrm{M} \mathrm{NaCl}$, and the differences in intensity of the substrate strands will be an effect of the reaction solution $\mathrm{NaCl}$ concentration. First, the activity was shown to be reduced at concen- 
trations below $50 \mathrm{mM} \mathrm{NaCl}$ ( $20 \mathrm{mM}$ and $0 \mathrm{mM} \mathrm{NaCl}$ ) because of decreased complex stability. In the $20 \mathrm{mM} \mathrm{NaCl}$ case, the uncleavable signal is increased, indicating an unstable complex, and resulting in fast release of substrate from the enzyme. While the $0 \mathrm{mM} \mathrm{NaCl}$ case does not show the increased uncleavable signal, it is likely due to the substrate already being significantly decreased from denaturing during the rinse step immediately prior to placement in the reaction solution. At concentrations above $50 \mathrm{mM} \mathrm{NaCl}(200 \mathrm{mM}$ and $1 \mathrm{M} \mathrm{NaCl}$ ), a stabilization of the complex was observed as evidenced by reduction of the uncleavable signal. The cleavable signal remained low, however, due to the stabilization of the 9mer cleaved fragment which does not separate from the enzyme strand.

The retention of $\mathrm{NaCl}$ dependencies of the $\mathrm{Pb}^{2+} \mathrm{DNAzyme}$, when moving from solution to surface, was reassuring because this sensing methodology is intended to be a general approach for sensing of analytes as future DNAzymes are identified. It was thus important to verify that specificity for the DNAzyme toward the analyte of interest was also retained. An NCAM-based specificity study was conducted to evaluate this aspect of the sensor (Figure 65). The results showed that while the immobilized DNAzyme complex was still most active in the presence of $\mathrm{Pb}^{2+}$, it lost some of its specificity, especially against $\mathrm{Zn}^{2+}$ and $\mathrm{Co}^{2+}$.

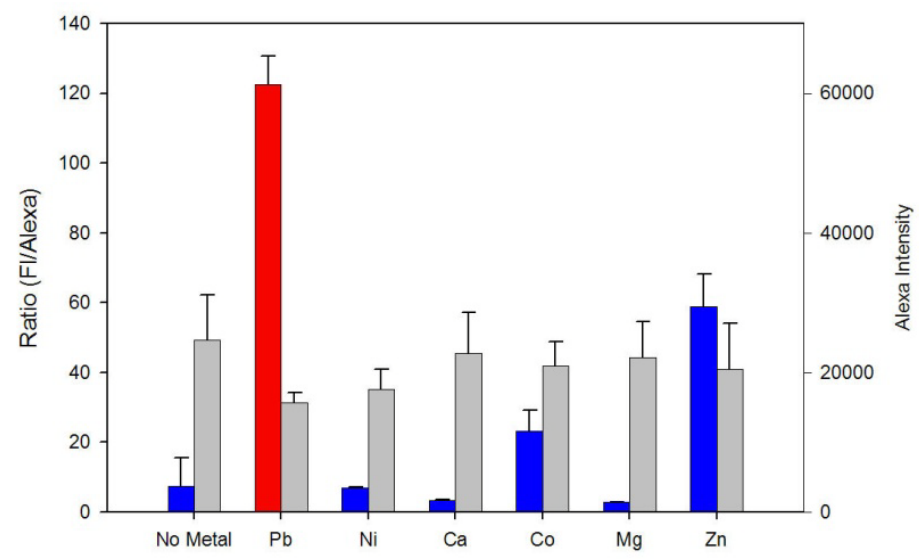

Figure 65. Specificity of $\mathrm{Pb}^{2+}$ DNAzyme on NCAM versus other divalent metal ions: $\mathrm{Pb}^{2+}$ shows highest activity, however some specificity of the DNAzyme is lost compared to the native 17E/17S system; uncleavable Alexa546 intensity is shown in gray, activity ratio (Fl/Alexa) in presence of $\mathrm{Pb}^{2+}$ in red and other divalent metal ions in blue. Error bars represent one standard deviation of three independent reactions.

There was immediate concern that the immobilization method had affected the specificity of the DNAzyme complex. However, a solution-based specificity experiment was performed to check the effect of modifying the 
original 17E sequence by extending the 5 ' enzyme arm and complementary substrate arm, adding a poly-T linker, and modifying with thiol (Figure 66). It was previously thought that modifications away from the metal binding arm and conserved bases would yield no effect on DNAzyme activity. Contrarily, it was observed that the arm modifications did alter the specificity of the DNAzyme in the solution based case. There was, however, no difference in specificity for the modified system in solution when compared to the surface immobilized system, showing the immobilization does not affect specificity.

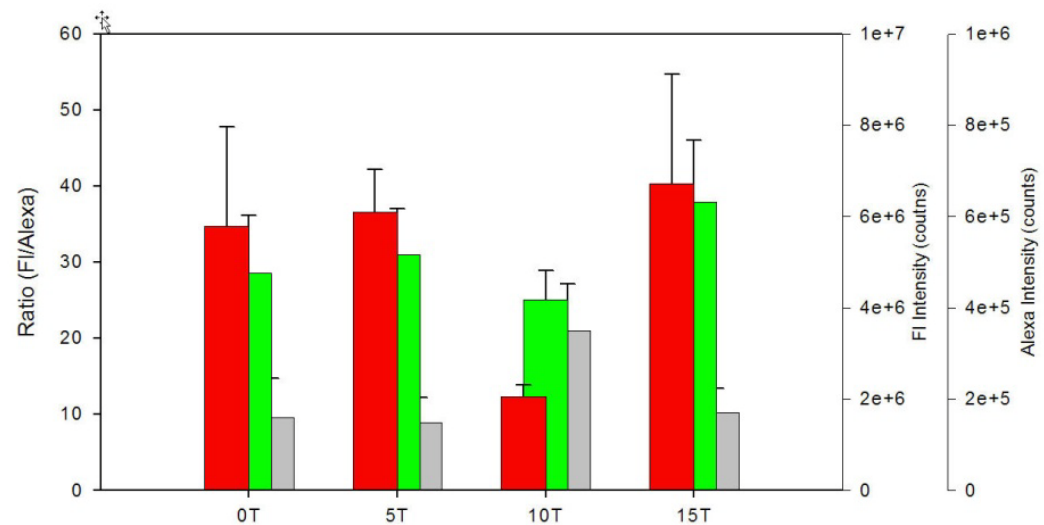

Figure 66. The effect on DNAzyme activity of enzyme poly-T linker length between 5 '-thiol and first complimentary base. While no dependence on linker length is observed for $0 \mathrm{~T}, 5 \mathrm{~T}$, or $15 \mathrm{~T}$, a drastic decrease in activity is observed in the 10T system, though the cause is undetermined. Uncleavable Alexa 546 intensity is shown in gray, cleavable fluorescein in green, and the ratio of Fl/Alexa in red. Error bars represent one standard deviation of four independent reactions.

\subsection{On-chip lead detection}

\subsubsection{Fluorescence on a PMMA microfluidic device}

A mild and effective method for the immobilization of DNAzyme on PMMA, involving the reaction between biotin-modified DNAzyme and streptavidin physisorbed on the PMMA surface, was used to immobilize DNAzymes on the walls of the detection channel of the device. Using a fluorophore-labeled enzyme and a quencher-labeled substrate DNA, the immobilized DNAzyme was shown to undergo hybridization and then cleavage in the presence of $\mathrm{Pb}^{2+}$. The changes in surface fluorescence intensity associated with these processes enable the detection of $\mathrm{Pb}^{2+}$ in aqueous solutions in a microfluidic device in which $\mathrm{Pb}^{2+}$ is electrokinetically injected from the separation channel to the detection 
channel through a nanocapillary array membrane of pore size $200 \mathrm{~nm}$, bringing together all previous aspects of our research.

Previous studies showed that surface immobilization does not affect the activity or specificity of the DNAzyme, and this strategy retains the important advantages accruing to performing analytical determinations with surface-bound reagents.

Fluorescence images of the detection channel of the PMMA microfluidic / nanofluidic device after immobilization of the DNAzyme (Bio-5T-17Ea(7)Alexa), hybridization and subsequent exposure to $\mathrm{Pb}^{2+}$ are shown in Figure 67. Compared to the unmodified channel (image A), the channel modified with fluorescently labelled enzyme strand (image B) showed a significant increase in fluorescence intensity which indicates effective immobilization of the fluorescent DNAzyme. The fluorophore in this case was photostable Alexa 488, used to resist the photobleaching encountered with fluorescein-tagged DNAzyme.

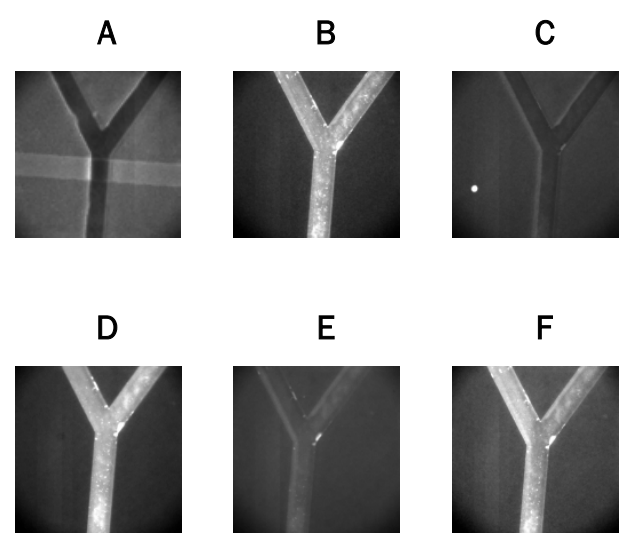

Figure 67. Study of activity of immobilized DNAzyme in the detection channel of the PMMA microfluidic-nanofluidic device: (A) unmodified channel; (B) immobilized enzyme strand; (C) after hybridization with quencher-bearing substrate strand; (D) after injection of $10 \mu \mathrm{M} \mathrm{Pb}^{2+}$ for $2 \mathrm{~h}$; $(\mathrm{E})$ after regeneration of channel with fresh substrate $\mathrm{DNA}$; and $(\mathrm{F}) \mathrm{Pb}^{2+}$ detection in the regenerated channel.

The images in Figure 67 confirmed that Alexa-tagged DNAzyme can be effectively immobilized in streptavidin-coated PMMA microchannels. After hybridization, the fluorescence is quenched (image $\mathrm{C}$ ) and upon exposure to $\mathrm{Pb}^{2+}$, the quencher-labeled substrate DNA is cleaved and released, and the fluorescence intensity increases again (image D). After detection of $\mathrm{Pb}^{2+}$ (image D), introduction of substrate DNA into the detection channel (image E) effectively hybridized the immobilized enzyme DNA, indicated 
by the fluorescence quenching within the channel. Repeated use for $\mathrm{Pb}^{2+}$ detection (image F) demonstrates the enzyme DNA remained stable and catalytically active for successive measurements. This successfully demonstrated a prerequisite for a multi-use sensor, requiring only an on-board supply of substrate DNA for regeneration.

This is the first time DNAzyme activity was demonstrated in the immobilized state in a PMMA device. Apart from being simple and effective, this immobilization strategy utilized milder conditions compared to covalent bonding, thereby avoiding damage to the fragile, thin PMMA layers in the microchannels. Our work with these immobilization methods and the behavior of the chip for lead determination was published in the prestigious scientific journal Lab on a Chip (Dalavoy et al. 2007).

Figure 68 shows the fluorescence images of the PMMA sheet $(0.125 \mathrm{~mm})$ : (A) after immobilization of DNAzyme, (B) hybridization, and (C) exposure to $\mathrm{Pb}^{2+}$ solution for $2 \mathrm{~h}$. The images showed that fluorescein-tagged DNAzyme can be effectively immobilized on streptavidin-coated PMMA. Even though the streptavidin was only physisorbed on PMMA, it adhered strongly and was not removed from the surface upon washing, thus leading to effective DNAzyme immobilization. After hybridization, the fluorescence is quenched only in the channel where the DNAzyme comes in contact with substrate DNA. Quenching does not occur in the area under the PDMS, though some quenching was observed that resulted from the leakage of solution outside the channel due to insufficient sealing between the PMMA and PDMS layers. On exposure to $\mathrm{Pb}^{2+}$, the quencher-labeled substrate DNA was cleaved and released, whereupon the fluorescence intensity in the channel increased again. The chip can also be regenerated after $\mathrm{Pb}^{2+}$ detection by flowing substrate DNA through the channel, and the regenerated surface can be used for $\mathrm{Pb}^{2+}$ detection without a significant decrease in efficiency. 
A

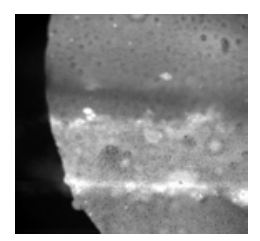

B

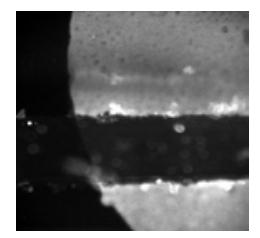

Figure 68. Fluorescence images of PMMA surface under the PDMS channel: Immobilized enzyme DNA spot where the channel is clearly observed passing through the spot (A); the spot after hybridization with substrate DNA tagged with quencher (B); and $\mathrm{Pb}^{2+}$ flow through the channel cleaves the substrate and results in a fluorescence increase (C).

\subsubsection{Glass microbeads for greater interaction}

We were concerned that the fluid flow dynamics allowed only a portion of $\mathrm{Pb}^{2+}$ in solution to reach and react with the immobilized DNAzyme surface. This mass transport limitation prevents much of the $\mathrm{Pb}^{2+}$ in solution from reaching our "detection zone."

One approach to reduce mass transport limitations involves immobilizing DNAzyme on beads that can be inserted in the microfluidic channel. This will provide a much higher surface area for interaction between DNA and $\mathrm{Pb}^{2+}$ solution. Immobilization of DNAzyme was done on $40 \mu \mathrm{m}$ glass beads. The reaction scheme and results are shown below (Figure 69 and Figure 70). Briefly, the beads were silylated and treated with glutaric dialdehyde to form terminal groups which were then reacted overnight with aminated DNA with a fluorescein label [Am(7)17E(-4)-Fl] (10 $\mu \mathrm{M}$ solution in $100 \mu \mathrm{M}$ phosphate buffer $\mathrm{pH} 7$ ). This was followed by a reduction of unreacted - $\mathrm{CHO}$ groups with $\mathrm{NaBH}_{4}$, washing, and drying. The modified beads show a fluorescent signal, indicating effective immobilization of DNAzyme. 


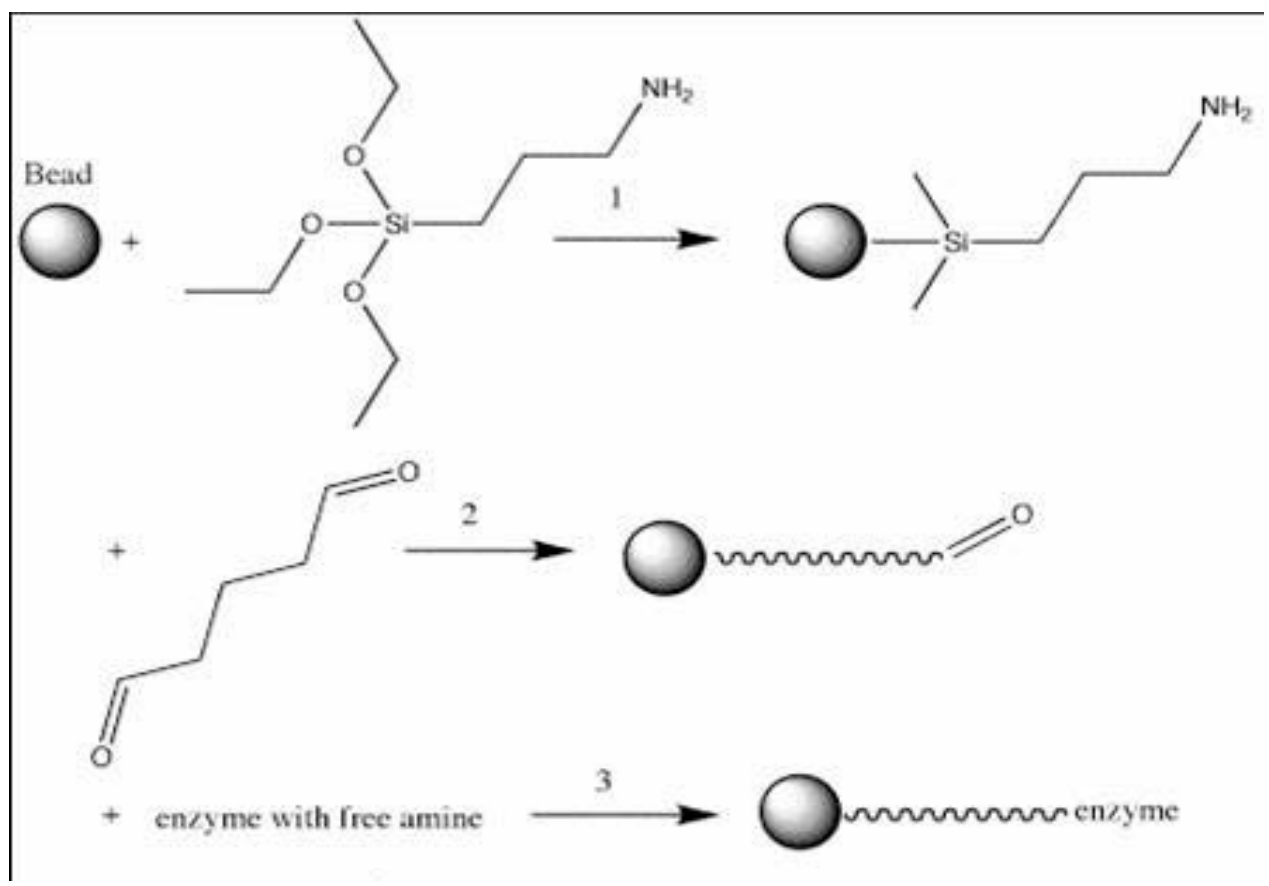

Figure 69. Scheme for immobilization of DNAzyme on glass microbeads.

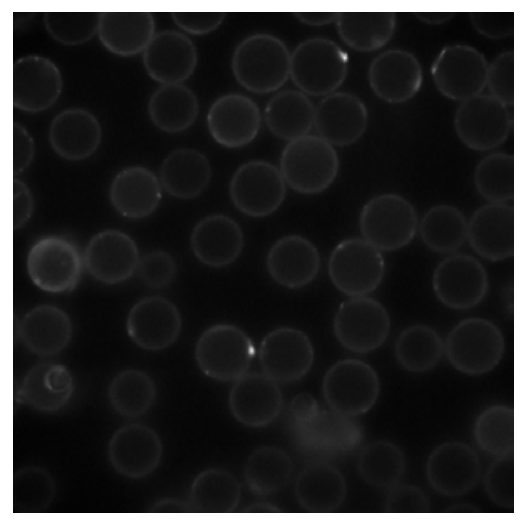

Unmodified glass beads
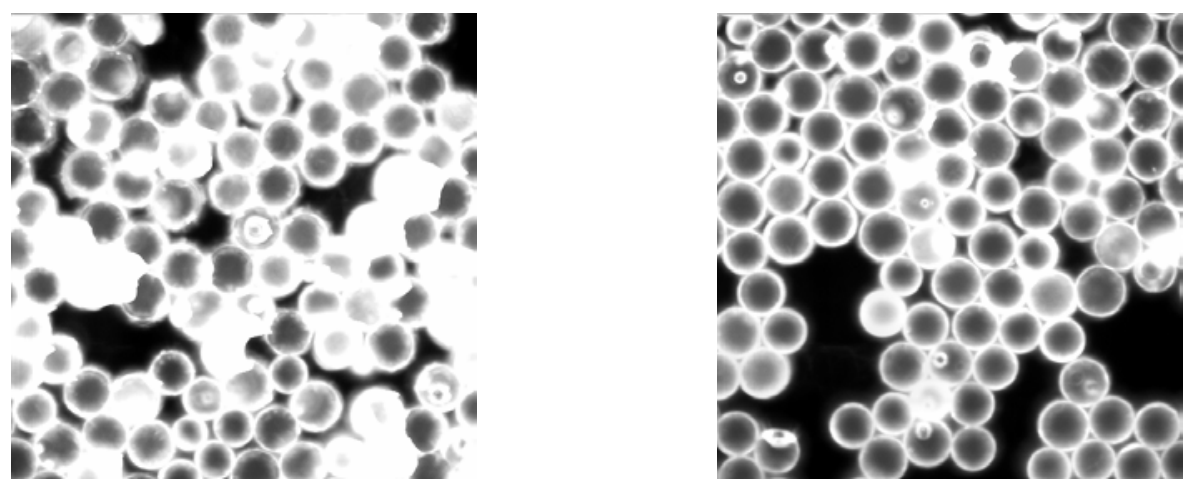

Am(7)17eA(-4)-Fl immobilized on glass beads

Figure 70. Fluorescence images of glass microbeads before and after DNA immobilization. 
In order to incorporate the beads in a microchannel, PDMS devices with a constricted channel were constructed using SU8 multilayering. The steps involved are shown in Figure 71. The PDMS device was constructed by pouring PDMS prepolymer and curing agent in the mold, followed by mixing, degassing, and curing at room temperature. The PDMS layer was plasma-bonded to the coverglass to make the complete device. A schematic representation of the device in side view is shown in Figure 72. The channel has a cross section of $100 \times 100 \mu \mathrm{m}$ for three-fourths of its length and $100 \times 20 \mu \mathrm{m}$ for one-fourth of its length. The $40 \mu \mathrm{m}$ microbeads were held in the $100 \times 100 \mu \mathrm{m}$ part of the channel; the constriction holds the beads in the channel while allowing only liquids to flow out of the other end.

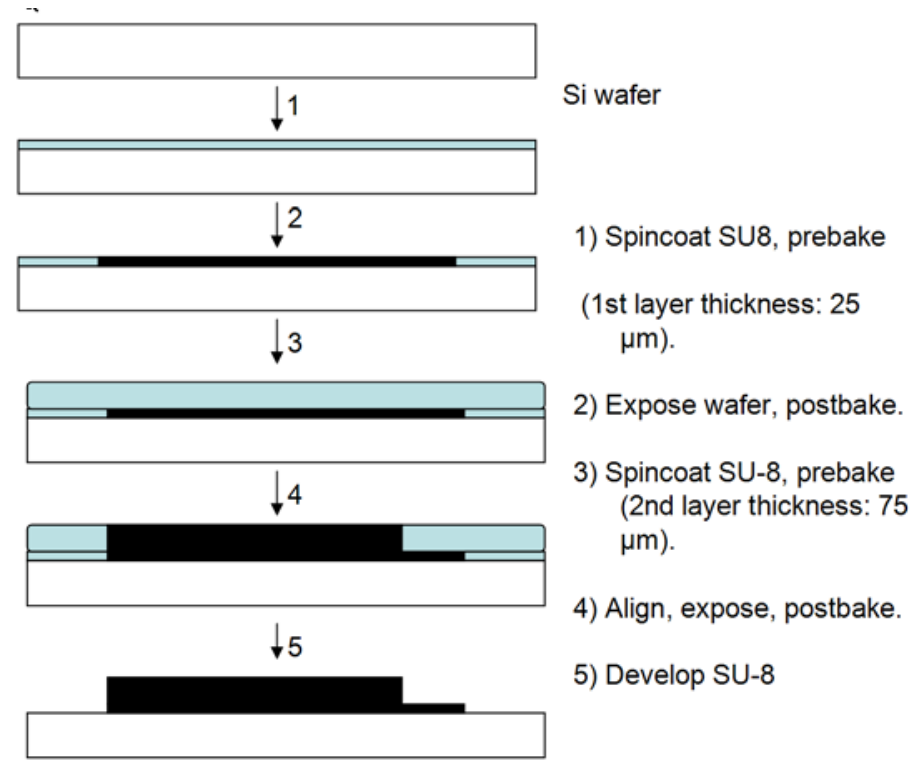

Figure 71. Steps involved in creating mold for constricted channel.

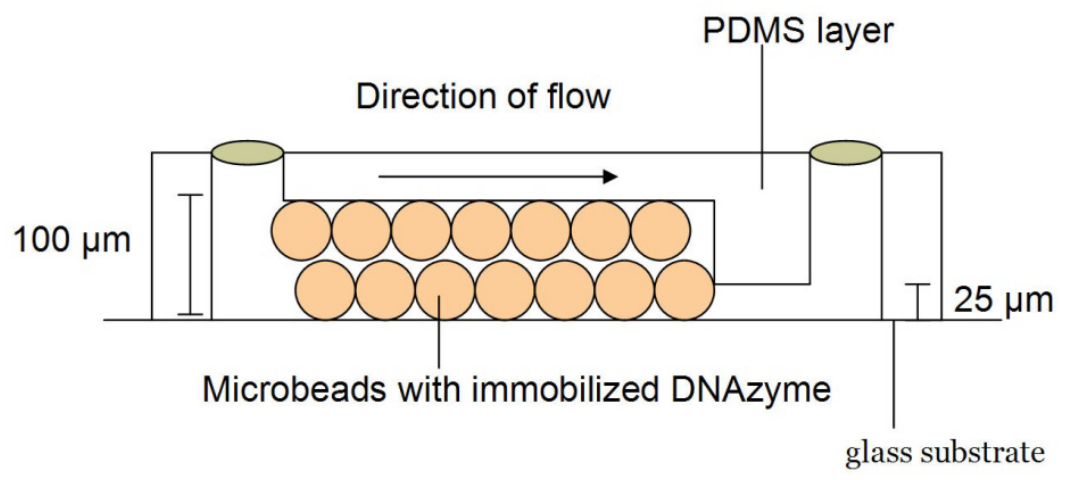

Figure 72. Side view of PDMS device with constricted channel. 
The DNA modified microbeads were suspended in DI water and introduced into the channel by adding the suspension to one reservoir and applying vacuum to the other reservoir. Hybridization with substrate DNA [IB(-4)17S(7)] was carried out overnight at room temperature, followed by flowing $10 \mu \mathrm{M} \mathrm{Pb}^{2+}$ solution for $2 \mathrm{~h}$. The fluorescence images after incorporation of beads in the PDMS channel, hybridization, and $\mathrm{Pb}$ reaction are shown in Figure 73. This work focused on quantification of the fluorescence signal with $\mathrm{Pb}^{2+}$ solutions at different concentrations. One challenge was the photobleaching of fluorescein which reduced the fluorescence signal and made accurate estimation impossible. This could be overcome by changing the fluorophore.

Immobilization

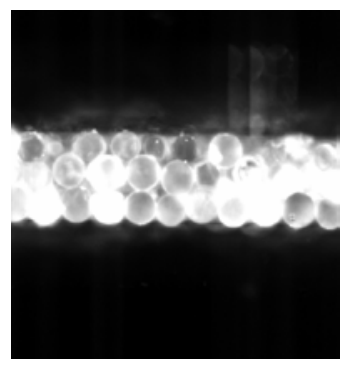

Figure 73. Fluorescence imag
Hybridization

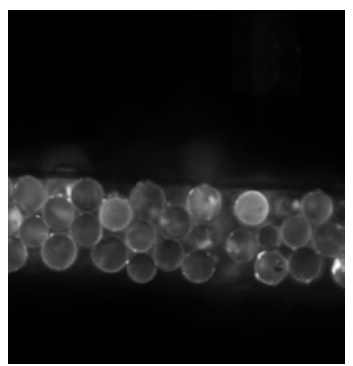

$\mathrm{Pb}$ reaction $\left(10 \mu \mathrm{M} \mathrm{Pb}^{2+}\right.$ for $\left.2 \mathrm{~h}\right)$ hybridization, and $\mathrm{Pb}$ reaction.

Due to the high rate of photobleaching observed with fluorescein-labeled DNAzyme, the beads were functionalized with Alexa-labeled DNAzyme. Due to the difficulty involved in synthesizing amine-modified Alexalabeled oligos, the functionalization method had to be changed to introduce biotin functionality on the glass beads by reacting the amine end groups with biotin N-hydroxy succinimide ester. Biotin-modified DNAzyme with Alexa label [Bio-5T(7)17DSa-Alexa 488] (10 $\mu \mathrm{M}$ in 1X Microspotting Solution Plus) was then introduced by incorporating the highly specific biotinstreptavidin-biotin interaction. The reaction scheme is shown in Figure 74. 


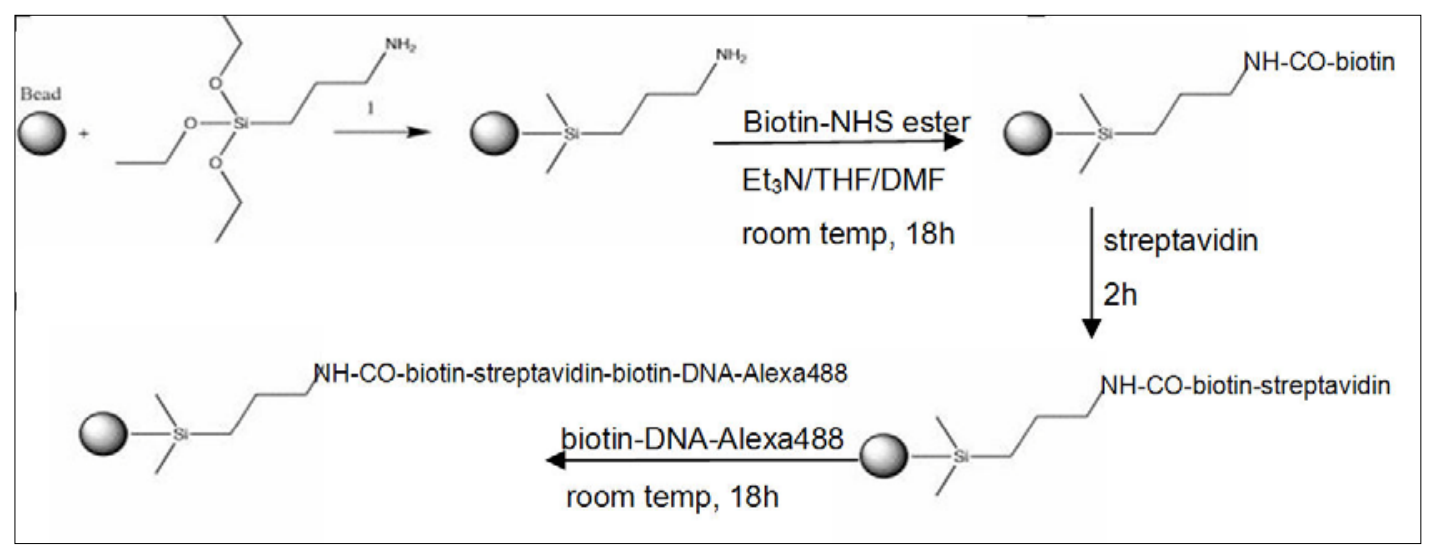

Figure 74. Scheme for immobilization of DNAzyme on glass microbeads.

The fluorescence images after immobilization of DNAzyme on the beads, hybridization, and $\mathrm{Pb}$ reaction are shown in Figure 75. These results showed the quenching of fluorescence on hybridization and an increase in fluorescence signal upon $\mathrm{Pb}$ reaction.

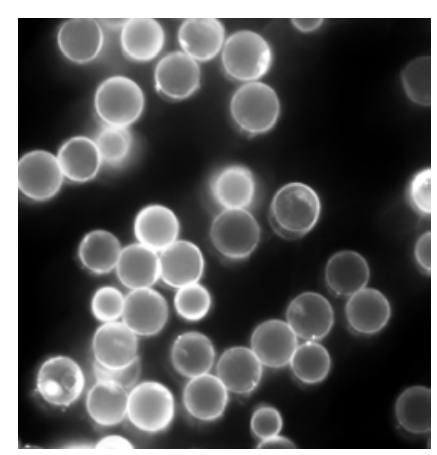

A

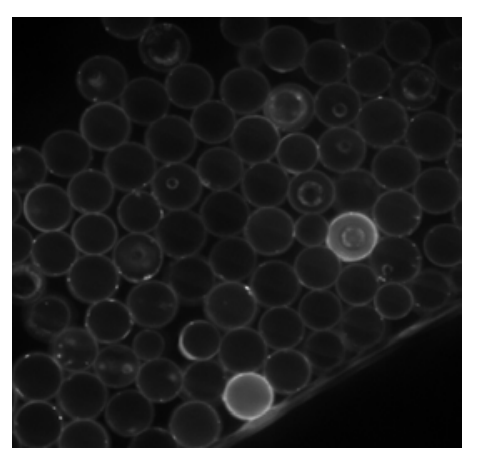

B

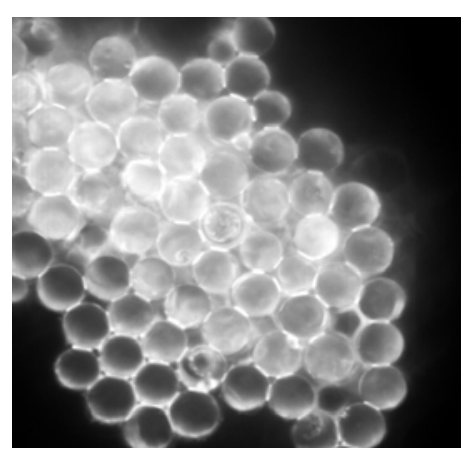

C

Figure 75. Fluorescence images of: glass microbeads after DNA immobilization (A), hybridization (B), and exposure to $10 \mu \mathrm{M} \mathrm{Pb}^{2+}(\mathrm{C})$

Once the activity of the functionalized beads was demonstrated, the DNAmodified microbeads were suspended in DI water and introduced into the PDMS/ glass devices with constricted channel by adding the suspension to one reservoir and applying vacuum to the other reservoir. Hybridization with $5 \mu \mathrm{M}$ substrate DNA [IB(-4)17S(7)] was carried out overnight at room temperature, followed by flowing of $\mathrm{Pb}^{2+}$ solution for $2 \mathrm{~h}$. The fluorescence images after incorporation of beads in the PDMS channel, hybridization, and $\mathrm{Pb}$ reaction at two different concentrations are shown in Figure 76. The results showed quenching of fluorescence upon hybridization and an increase in fluorescence signal upon $\mathrm{Pb}$ reaction. However, the fluores- 
cence signal is not quantitative, and it is difficult to distinguish between the two different $\mathrm{Pb}^{2+}$ concentrations as can be seen in Figure 76.

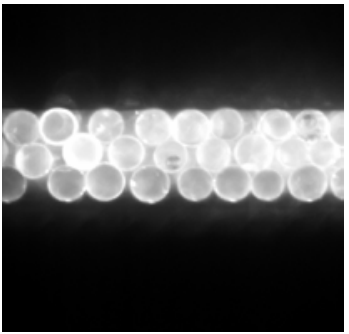

A

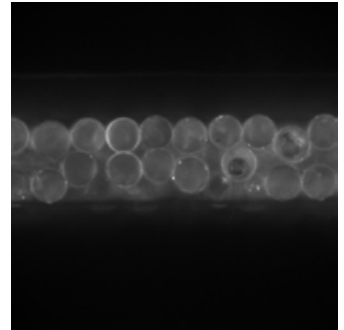

B

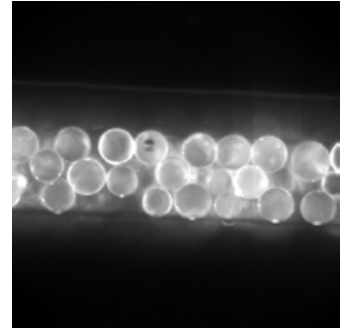

C

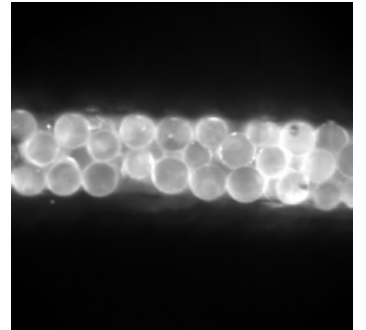

D

Figure 76. Fluorescence images after: incorporation of beads in the PDMS channel (A), hybridization (B), exposure to $10 \mu \mathrm{M} \mathrm{Pb}^{2+}(\mathrm{C})$, and $100 \mu \mathrm{M} \mathrm{Pb}^{2+}(\mathrm{D})$.

Therefore, the absence of a reference may pose a challenge for quantification of the fluorescence signal. In this case, Alexa 488 also showed a high rate of photobleaching due to the higher surface area of exposure to solution, but this could be minimized by degassing the buffer solution prior to use and reducing the exposure time after fluorescence measurement. The other challenge was the release of cleaved substrate DNA, which is much more difficult in this case and may be hindered by the closely packed beads. Due to this challenge, the increase in fluorescence signal was not uniform in beads from different parts of the channel. All these factors need to be addressed in order to achieve accurate determination of $\mathrm{Pb}^{2+}$.

Once effective immobilization, hybridization, and $\mathrm{Pb}$ reactions were demonstrated on each of the substrates, work focused on calibration of the fluorescence signal by using standard solutions of $\mathrm{Pb}^{2+}$, quantification of $\mathrm{Pb}^{2+}$ in aqueous solutions, and determination of analytical figures of merit. The substrates and immobilization techniques used in this study were ones that can be readily incorporated into PMMA microfluidic devices for sensor development.

This device provides a simple way to test the activity of DNAzyme towards $\mathrm{Pb}^{2+}$ sensing, all within a microchannel. It is also an important step toward detecting multiple heavy metal ions with a single injection by introducing different DNAzymes at different locations in the detection channel on a microfluidic-nanofluidic molecular gated device. 


\subsection{Electrochemical detection of DNAzyme activity}

Having shown fluorescent-based detection from NCAMs, our work moved toward the possibility of electrochemical detection of DNAzyme activity. Incorporation of Au-coated NCAMs into microfluidic devices provided both a surface ideal for immobilization of the DNAzyme, and the opportunity to use the conductive surface for electrochemical measurements. We have attempted to characterize the use of a redox-labeled DNAzyme substrate strand for electrochemical detection of $\mathrm{Pb}^{2+}$-induced activity. Electrochemistry of the DNAzyme at the membrane surface would allow use of the same electrical inputs as would be used for capillary separation and electrokinetic fluid flow within the microfluidic device, thereby alleviating the need for a photon detector. Furthermore, electrochemical detection of metal ions by DNAzymes should provide better detection limits compared to standard electrochemical detection of ions.

Our first work was to verify that DNAzyme activity is not lost during the application of potential from $-0.2 \mathrm{~V}$ to $+0.6 \mathrm{~V}$, the potential scan region for detection of a ferrocenyl-labeled oligonucleotide. Using fluorescently labeled DNA, we engineered DNAzyme immobilized Au-NCAMs into electrodes that could be used for electrochemistry. Using a potentiostat to apply voltage through the specified range, we observed no induced dehybridization of assembled DNAzyme complex nor loss of DNAzyme activity. This proved that the NCAMs could be used for potential electrochemical application without destructively affecting the DNAzyme.

We then moved to conjugating a ferrocene label to 3'-amine modified substrate DNA via 1-ethyl-3-(3-dimethylaminopropyl)-carbodiimide (EDC) coupling. Following literature reported methods, we were able to purify the ferrocenylated substrate strand. Immobilization and hybridization for formation of the DNAzyme complex on gold disc electrodes allowed for electrochemical detection of the ferrocene label in a buffer solution. For electrolyte concentrations high enough to provide quality electrochemical signals, it was necessary to increase sodium perchlorate concentration to $400 \mathrm{mM}$, thereby increasing sodium ion content from the $50 \mathrm{mM}$ ideal activity concentration to $400 \mathrm{mM}$, which inhibited release of the ferrocene label. This required truncation of the DNAzyme release arm by four bases to provide reasonably fast release of cleaved product. Activity of the truncated DNAzyme was verified by fluorescent assay. We have shown the ability to detect the presence of $\mathrm{Pb}^{2+}$ using the DNAzyme by electrochemical detection on gold disc electrodes. This is shown in preliminary data in Fig- 
ure 77. The cyclic voltammograms of the ferrocenylated-substrate strand shows near an $80 \%$ loss of signal after 10 min of reaction with $10 \mu \mathrm{M} \mathrm{Pb}^{2+}$ as the ferrocenylated fragment releases from the DNAzyme, while control samples with no $\mathrm{Pb}^{2+}$ showed less than $10 \%$ loss over the same period. The current sensor design is "light-down" for proof of concept, but other design concepts could provide a "light-up" sensor. We now look to move to the Au-NCAM surfaces for electrochemical detection of DNAzyme activity.
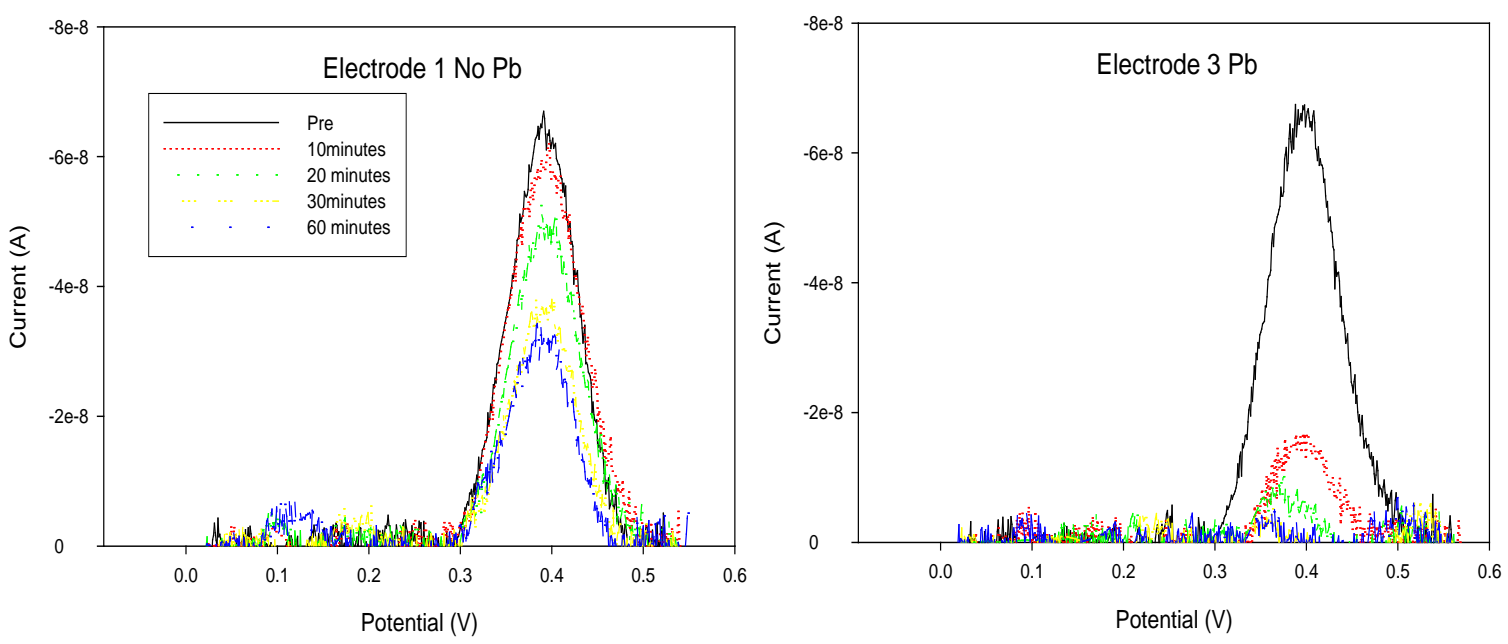

Figure 77. Baseline-corrected ferrocenyl anodic peaks from cyclic voltammograms scanned from $0.0 \mathrm{~V}$ to $0.6 \mathrm{~V}$ showing $\mathrm{Pb}^{2+}$-DNAzyme activity. After 10 min of reaction with $10 \mu \mathrm{M} \mathrm{Pb}^{2+}$, signal loss is $\sim 80 \%$ compared to less than $10 \%$ in the sample that has no $\mathrm{Pb}^{2+}$.

\subsection{Immobilization of DNAzyme on fluorous-coated silica in PDMS channel}

One final method for DNA attachment to surfaces was also briefly studied. Our goal was to fashion an immobilization step for DNAzyme that is usercontrollable (i.e., we can immobilize and strip off the DNAzyme for better quantification of fluorescence at a single detection zone), and we can regenerate the reaction zone with fresh DNAzyme. Reversible immobilization of DNAzyme was carried out on fluorous silica packed in a microchannel formed with PDMS on glass, followed by hybridization, and then the lead reaction. After washing with buffer, the immobilized DNAzyme can be stripped off the surface by using methanol, and the fluorescence can be detected in solution since the hybridization state of DNA was found to be unaffected by the solvent. This method is consistent with our goal of having a lead reaction and detection zone separated to enhance detection efficiency. This approach should reduce mass transport limitations and improve accuracy since it will eliminate errors due to insufficient 
release of substrate DNA after $\mathrm{Pb}^{2+}$ reaction and cleavage, as observed during $\mathrm{Pb}^{2+}$ detection in the microchannel. Any substrate DNA nonspecifically bound to the enzyme in the channel would be eluted along with the enzyme and then released in solution. Photobleaching is not a problem because the fluorous silica surface can be regenerated with fresh fluorescent DNA after each measurement. This method also eliminates problems involved in surface fluorescence detection such as non-uniformity of signal intensities on the surface, uncertainty in sampling points, and poor reproducibility. Obtaining standards for comparison of fluorescence signal should not be difficult in this case. The fluorescence intensity from the sample eluted after hybridization and flowing buffer in the absence of lead should account for the background fluorescence; the fluorescence intensity from the denatured samples should provide an estimate of total DNA immobilized, and photobleaching effects and the actual intensities obtained after flowing various concentrations of lead can be referenced against this value. Further, we can chemically denature the stripped DNAzyme sample and the subsequent fluorescence intensity from the denatured samples would provide an estimate of total DNA immobilized and photobleaching effects. Lead signals can be normalized against this value.

The results of our initial studies using this approach are shown in Figure 78. Images show fluorous silica packed in the microfluidic channel, modified with fluorescein-labeled $\mathrm{Pb}$-specific catalytic DNA, quenching of fluorescence on hybridization, increase in fluorescence signal on exposure to $\mathrm{Pb}^{2+}$, removal of DNAzyme on elution, and regeneration of the fluorous silica with fresh DNAzyme. All parts of the $\mathrm{Pb}^{2+}$ detection process with respect to the fluorous silica immobilization, hybridization, and reaction work as expected. The detection process for the lead concentration can now occur downstream from the fluorous silica. 


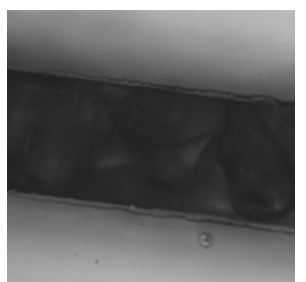

Fluorous silica in PDMS channel (100 um channel)

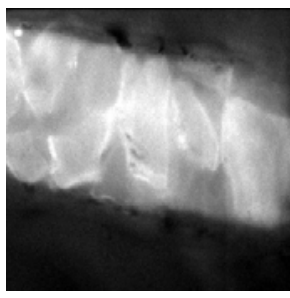

$\mathrm{Pb}$ reaction (10 $\mu \mathrm{M}$ for $2 \mathrm{~h}$ )

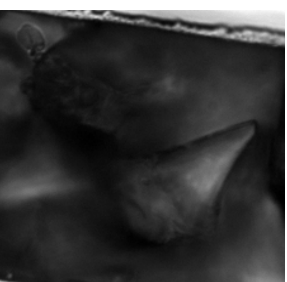

Expanded View

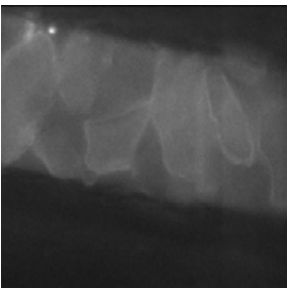

Elution with $100 \%$ $\mathrm{MeOH}$

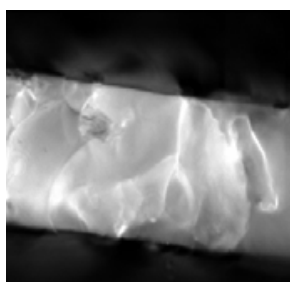

Immobilization of DNAzyme

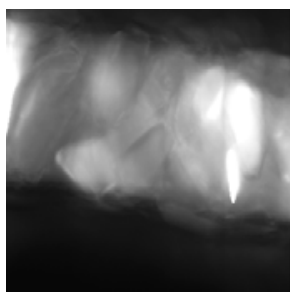

Reload with DNAzyme after regeneration with THF

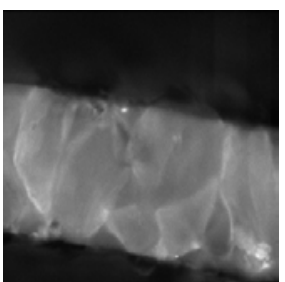

Hybridization

Figure 78. From top left to bottom right: immobilization of DNAzyme and subsequent $\mathrm{Pb}^{2+}$ detection on fluorous-coated silica packed in a PDMS channel.

Fluorescence intensities of eluent obtained after exposure to $10 \mu \mathrm{M}$ and $100 \mu \mathrm{M} \mathrm{Pb}^{2+}$ solutions are shown in Figure 79. The data show only an increase in background instead of a fluorescence peak, which indicates that the fluorophore concentration may be below the detection limit of the fluorimeter. In this case, the eluent was diluted to $500 \mu \mathrm{L}$, which is the minimum volume required for the cuvette used in the fluorimeter. For this process to work more effectively, we believe a different approach involving on-chip concentration of the eluent and detection in a small volume will solve this problem. Another option would be increasing the amount of fluorous silica in the channel to increase the amount of DNAzyme present, but this would cause high flow resistance. At this point, we have shown this immobilization scheme to have potential. 

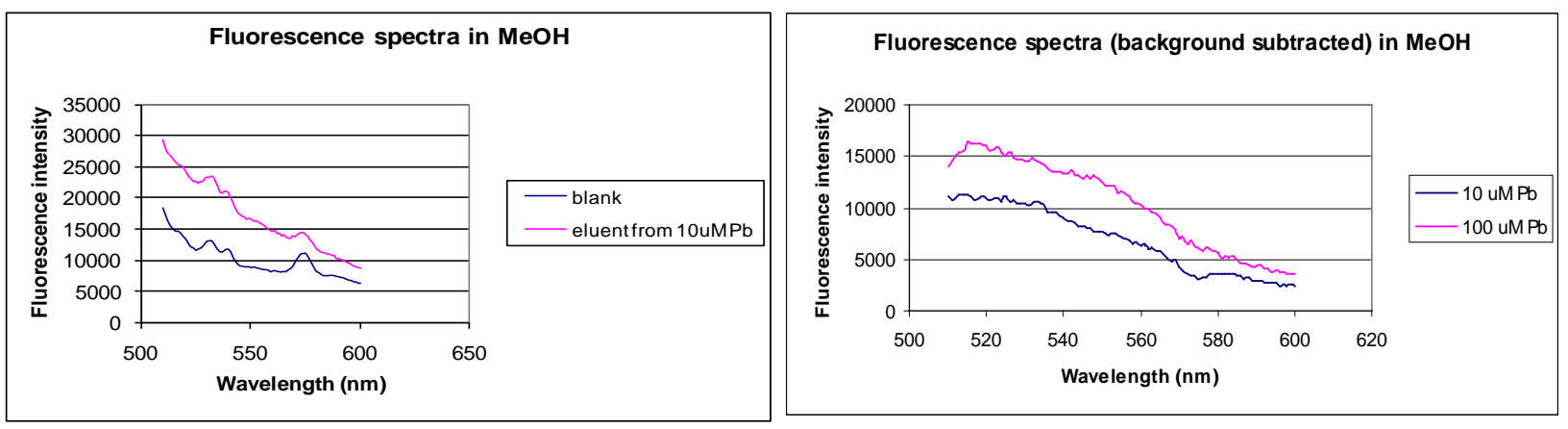

Figure 79. Fluorescence spectra of eluent from channel after exposure to $\mathrm{Pb}^{2+}$.

\subsection{A new DNAzyme specific for uranium}

As part of this general project, we proposed developing a microfluidic platform that can demonstrate multiplexing behavior; that is, show that the platform is capable of detecting multiple analytes with a single injection. A requirement for this task is a second cation-specific DNAzyme that can be incorporated onto the device, together with our current lead-specific DNAzyme.

To this end, we successfully selected for a DNAzyme for the uranyl ion, $\mathrm{UO}_{2}{ }^{2+}$, which is the stable form of uranium in groundwater. Figure 80 shows the structure and sensing parameters for the $\mathrm{UO}_{2}{ }^{2+}$-specific DNAzyme. It transduces the presence and concentration of $\mathrm{UO}_{2}{ }^{2+}$ in water via the same fluorosensing mechanism as the lead-specific DNAzyme (i.e., a fluorophore on the substrate DNA strand is in close proximity to a quencher on the enzyme DNA strand) as show by image C in Figure 80. In this hybridized state, the fluorescence is quenched. Upon reaction with the $\mathrm{UO}_{2}{ }^{2+}$, the substrate is cleaved, fluorophore and quencher are separated, and the fluorescent signal is enabled where the intensity is proportional to the $\mathrm{UO}_{2}{ }^{2+}$ concentration.

This particular DNAzyme has an extremely low detection limit of 11 ppt for the uranyl ion and would be invaluable for military use in environmental detection of depleted uranium. This achievement was published in Proceedings of the National Academy of Sciences of the United States of America (Liu et al 2007) and allowed us to proceed with the creation of a multianalyte detection device. 


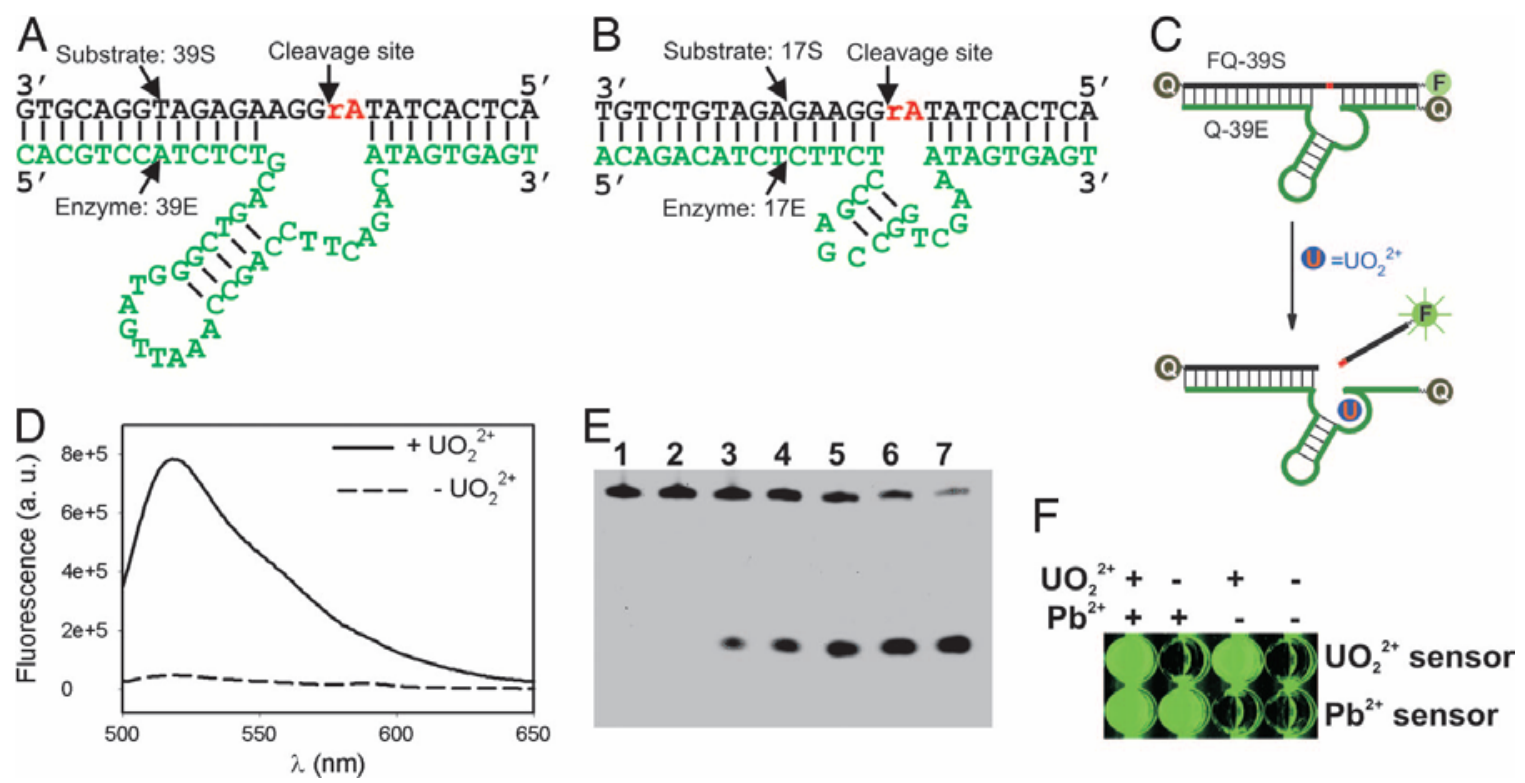

Figure 80. (A) Structure of the $\mathrm{UO}_{2}{ }^{2+}$ specific DNAzyme showing the top substrate strand and the bottom enzyme strand. (B) The lead-specific DNAzyme structure for comparison. (C) The fluorosensing scheme. (D) The fluorescent response of the $\mathrm{UO}_{2}{ }^{2+}$ specific DNAzyme after 10 min. In the absence of $\mathrm{UO}_{2}{ }^{2+}$, fluorescence at $525 \mathrm{~nm}$ remains completely quenched. In the presence of $\mathrm{UO}_{2}{ }^{2+}$, the fluorescein label is visible. (E) The response of the substrate DNAzyme without the enzyme (lane 1), and the response of the total construct in lanes $2-7$ after 0,1 ,

$2,5,10$, and 30 min reaction with $\mathrm{UO}_{2}{ }^{2+}$. (F) A sensor array containing both $\mathrm{UO}_{2}{ }^{2+}$ and $\mathrm{Pb}$ sensors demonstrating the potential for multiplexing.

\subsection{Multianalyte device: detection of lead and uranium ions in a PDMS/glass microfluidic device}

We then examined the possibility of a multianalyte device using not only the lead-selective DNAzyme but also the uranyl $\left(\mathrm{UO}_{2}{ }^{2+}\right)$-selective DNAzyme described earlier (Liu et al. 2007). The ability to detect lead and uranium separately -in ground water, in the same injection, on the same device-would represent a significant advancement in portable microfluidic devices for environmental analysis. These analytes are both highpriority Army contaminants.

In order to demonstrate detection of multiple analytes in a single microfluidic channel, the biotinylated DNAzymes for $\mathrm{Pb}^{2+}$ [Bio-5T(7)17EaAlexa488] and $\mathrm{UO}_{2}{ }^{2+}$ [Bio-39E-Alexa488] were immobilized in separate spots on streptavidin-coated glass. The DNAzymes were modified with biotin, which interacts with streptavidin, and each was labeled with a fluorophore. After blocking unreacted streptavidin and washing, a PDMS layer with a single channel was placed on the surface such that the channel intersected each spot. The DNAzyme spots were then hybridized by flowing quencher-labeled substrate DNA through the channel. This effectively 
quenched the fluorescence of the immobilized DNAzyme which then appears as a dark horizontal stripe through each spot. Finally, $10 \mu \mathrm{M}$ solutions of $\mathrm{Pb}^{2+}$ or $\mathrm{UO}^{2+}$ were electrokinetically driven through the channel using a $50 \mathrm{~V}$ applied potential. The results are shown in Figure 81. Enzyme $17 \mathrm{E}$ and substrate 17S are the lead-selective DNA enzyme and substrate, respectively, while 39E and 39S respond to the uranyl ion. 


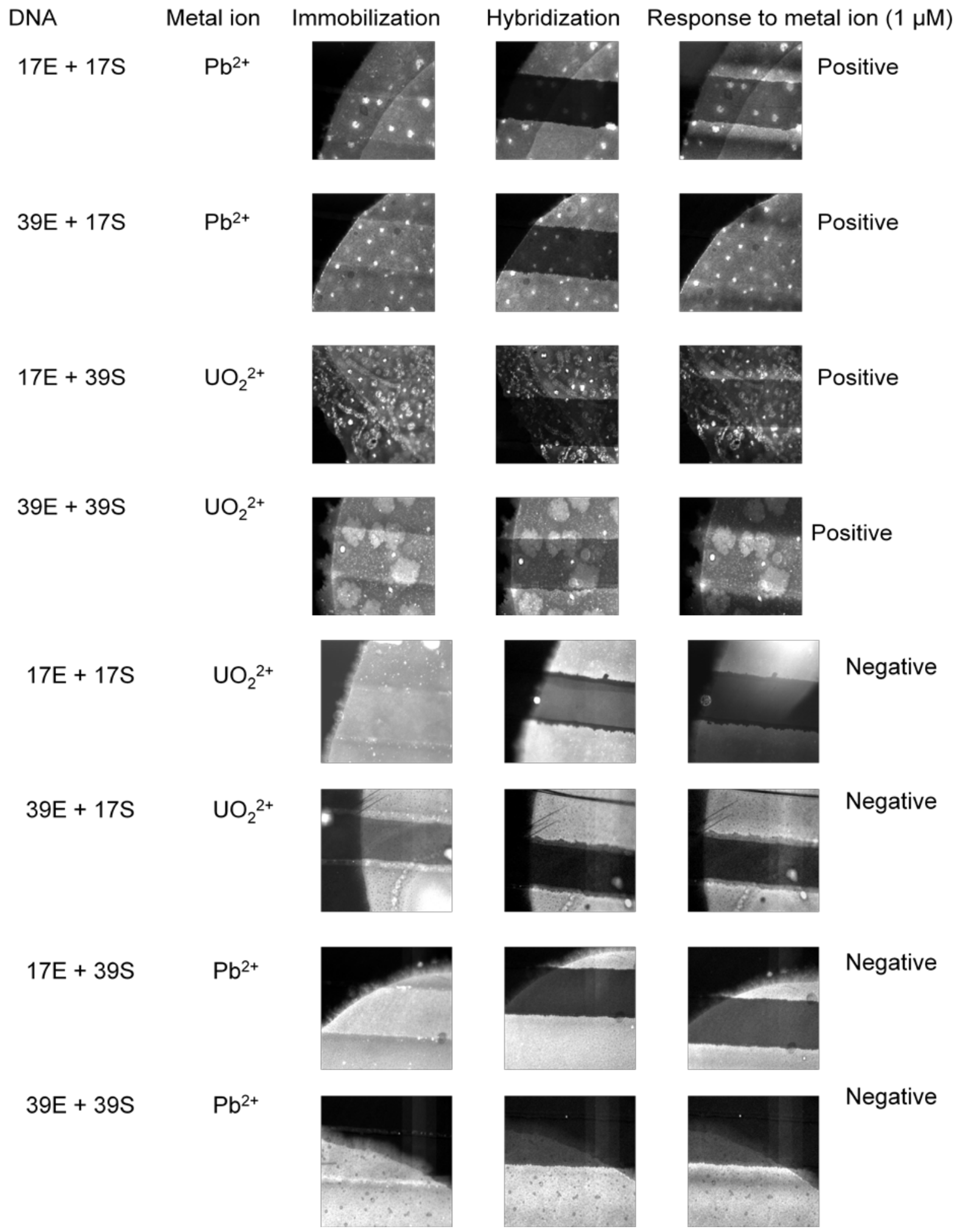

Figure 81. Fluorescence images showing spots with various combinations of enzyme and substrate DNA and metal ions. 
From the results, it was found that the combination 17E+17S responds as expected to $\mathrm{Pb}^{2+}$, as noted by the increase in fluorescence. This combination does not respond to the uranyl ion. Also, combination 39E+39S did respond to $\mathrm{UO}_{2}{ }^{2+}$ and does not respond to $\mathrm{Pb}^{2+}$, behaving exactly as it should. It is the other combinations of enzyme with substrate that are interesting. The substrate sequences $17 \mathrm{~S}$ and 39S are very similar; each will hybridize to either of the enzyme strands 17E or 39E. Sequence 39E+17S has a negative response to $\mathrm{UO}_{2}{ }^{2+}$ (as expected), but a positive response to $\mathrm{Pb}^{2+}$. Conversely, sequence 17E+39S has a negative response to $\mathrm{Pb}^{2+}$ (as expected), but a positive response to $\mathrm{UO}_{2}{ }^{2+}$. This seems to imply that if the substrate strand matches its metal analyte, the enzyme will cleave it. Since the substrate strands are able to hybridize either enzyme, and since the substrate strand dictates its reactivity, the spots result in non-specific responses to these metal ions. Clearly, the DNAzyme spots for the two metals need to be isolated in different channels and processed separately. A theoretical device design with this capability is shown in Figure 82. The valving system allows selection of the direction of the hybridization solution to a particular detection channel and further allows direction of the water sample for analysis.

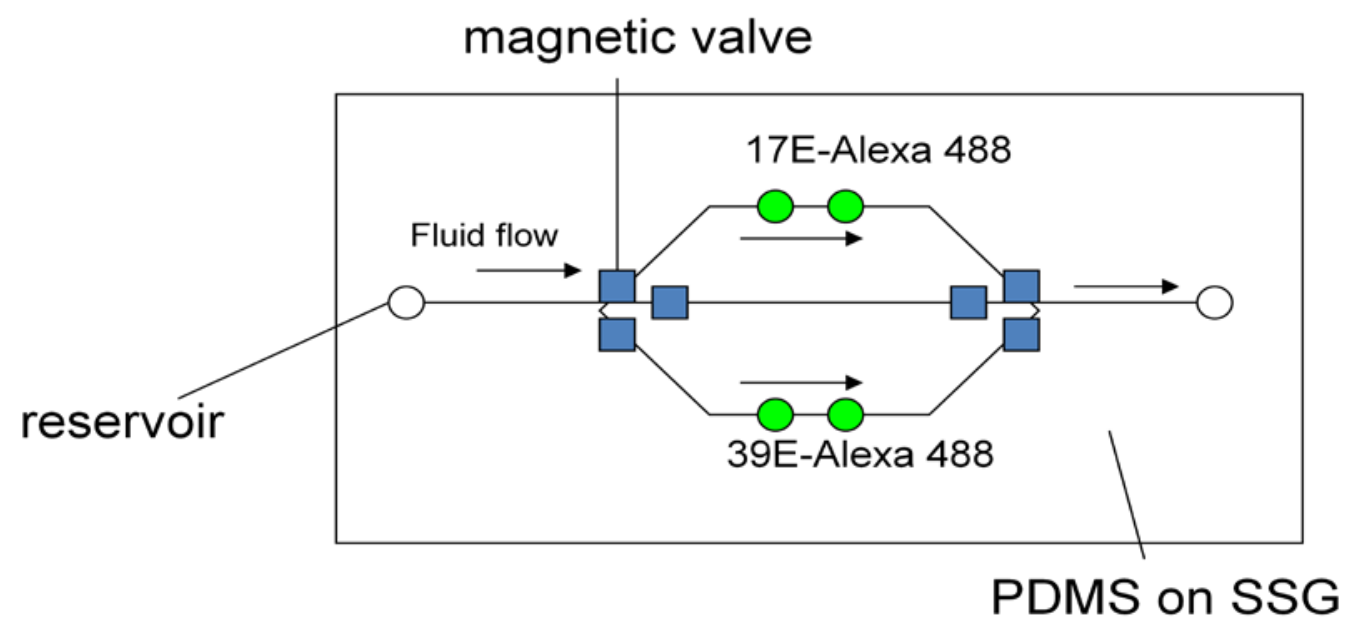

Figure 82. Device design for multianalyte (lead and uranium) analysis on a single injection. 


\section{Conclusions and Implications for Future Work}

\subsection{Conclusions}

This work demonstrates the feasibility of (1) incorporating $\mathrm{Pb}^{2+}$-specific catalytic DNA in a polymer-based microfluidic device and (2) its application for the detection of $\mathrm{Pb}^{2+}$. Using a fluorophore-labeled enzyme and a quencher-labeled substrate DNA, the immobilized DNAzyme was hybridized with a substrate DNA strand which then initiated cleavage in the presence of only $\mathrm{Pb}^{2+}$. The changes in surface fluorescence intensity associated with these processes enabled the detection of $\mathrm{Pb}^{2+}$ in aqueous solutions in a microfluidic device in which $\mathrm{Pb}^{2+}$ is electrokinetically injected from the separation channel to the detection channel through a nanocapillary array membrane of $200 \mathrm{~nm}$ pore size. The device is effectively regenerated by hybridization of the immobilized DNAzyme and can be repeatedly used for $\mathrm{Pb}^{2+}$ detection without significant reduction in fluorescence signal, thus reflecting the robustness of the design. The selectivity of the DNAzyme towards $\mathrm{Pb}^{2+}$ detection was also retained in the immobilized state in the microfluidic device. The presence of $\mathrm{Pb}^{2+}$ can be detected without interference from other metal ions present in the sample. This is the first time DNAzyme activity was demonstrated in the immobilized state in a PMMA device. Apart from being simple and effective, the immobilization strategy we utilized has milder conditions as compared to covalent bonding, thereby avoiding damage to the thin PMMA layers in the microchannels.

Immobilization of catalytic DNA directly on the PMMA surface, instead of the Au-NCAMs, enabled the analysis of lead by the measurement of surface fluorescence using fluorescence microscopy. This type of immobilization also eliminated potential problems of high background fluorescence of the Au surface and difficulties inherent in inserting metallic structures into polymeric microfluidic constructs. This immobilization strategy was compatible with multidimensional lab-on-a-chip schemes where microfluidic separations would be coupled to advanced processing methods with detection occurring at unique locations. 
Our research in this program also uncovered other scientific advances secondary to our primary goal of on-chip lead detection using DNAzymes.

The ability to create microfluidic devices in PMMA represents a significant advance for materials engineering. Solving the problem of bonding PMMA to the polycarbonate membranes created leak-free devices with the capability to introduce nanofluidic membranes on the chip at any desired location. Furthermore, use of PMMA provided a level of electrophoretic control unavailable on PDMS devices and without the problem of absorption of analyte into the channel material. We now have the capability to make devices in either polymer and with any desired level of complexity and analyte control.

We showed that separations could be done on either type of polymeric device, PMMA or PDMS. We showed that movement and separation of fluorescently labeled amino acids on PMMA can be observed. We also showed the analyte of interest could be extracted and sent to another channel, illustrating excellent analyte control and reproducible timing.

We developed and proved DNAzyme immobilization on gold surfaces, PMMA, silica particles, superaldehyde glass, and superstreptavidin glass. Also, by coating the internal surface of the nanopores with gold, we could attach the DNAzyme within the membrane pores to create attoliter reaction chambers. This was shown by labeling the DNAzyme with a fluorophore or quencher and observing the fluorescent signal change as a result of washing, hybridization, and cleaving reactions.

This result was extremely important since it allowed us to immobilize the enzyme strand anywhere on the device and establish the optimal reaction zone while preserving and reusing the enzyme for further measurements. Desired areas or structures within the channels could be gold coated followed by a reaction with thiolated DNAzyme. PMMA and glass substrates could be coated to give a channel whose entire wall is an active sensing structure. We used this information to incorporate the immobilized DNAzyme within a PDMS device. Finally, the ability to immobilize a particular DNAzyme at a specified location enables the development of a multianalyte device with unique placement of multiple types of DNAzyme.

Finally, we also answered questions regarding the activity and regeneration of immobilized DNAzymes. The substrate DNA strand was able to hy- 
bridize to DNAzymes immobilized on gold substrates, indicating that we are not sterically hindered and the DNAzyme does not show non-specific bonding to the gold. This hybridized structure remained active toward lead in solution thereby illustrating that this sensing module can be utilized in an immobilized state on the device. We further demonstrated that the bound DNAzyme can be regenerated with new substrate strand solution for a further round of lead sensing, leading to the conclusion that this sensing modality can be used in an immobilized state and can be regenerated for continual use.

This work has been quite successful, with much of the scientific and technical output reported through a number of peer-reviewed publications in high-impact factor journals (Appendix B).

\subsection{Future work}

Future work could include the following tasks that revolve heavily around the engineering aspects of the project:

1. Address the concern for stability and robustness of this DNAzyme under realistic environmental conditions. In the laboratory at $4{ }^{\circ} \mathrm{C}$, the hybridized construct was stable for months. With increasing ambient temperatures, denaturation of the construct becomes more problematic with a general concomitant increase in background fluorescence. First, it must be mentioned that one researcher involved in this project (Lu) has been extensively studying the relationship between the number of Watson-Crick base pairs in the binding regions and the effective temperature sensing regime. The effective temperature regime can be very tightly controlled. This parameter, however, should be studied carefully when the DNAzyme is bound within a microfluidic device. Second, there is also a concern about natural enzymatic, bacterial, or UV degradation of the DNA strands. With careful consideration to the design features of the device, we can eliminate most of these issues with (a) filtration that only passes molecular size analytes, (b) timing of fluidic transport control for introduction of only lead into the detection channel, and (c) enclosure of the device for light isolation of the detection channel.

2. Immobilize both $\mathrm{Pb2}+$ and UO22+-specific DNAzyme on SSG, followed by hybridization and exposure to a mixture of the two cations. The two DNAzymes may be tagged with similar or different fluorophores for multiplexed detection and selectivity and interference 
studies should be carried out. The ultimate goal is to detect and quantify the two cations in the same microfluidic device, but in different channels since our previous studies have indicated cross-hybridization due to similarity in the substrate DNA sequences for the two cations.

3. Solve the problem of device-to-device variability, which affects quantification of Pb2+in PDMS-SSG devices, by solving the problem of photobleaching so that repeated measurements can be carried out in the same device. For this purpose, instead of Alexa-labeled enzyme, we suggest that a quantum dot-labeled DNAzyme be used along with a FRET-based method with Texas Red-labeled substrate DNA to quantify lead.

4. Reversible immobilization of DNAzyme on fluorous-coated glass cover slips by using fluorous interaction, followed by binding a PDMS layer with a single microchannel, hybridization, and lead reaction. After washing with buffer, the immobilized DNAzyme can be stripped off the surface using methanol, and the fluorescence detected in solution after dilution of the sample as required, since the hybridization state of DNA was found to be unaffected by the solvent. This method is consistent with our goal of having the lead reaction and detection zones separated to enhance detection efficiency. This approach should reduce mass transport limitations and improve accuracy, since it will eliminate errors due to insufficient release of substrate DNA after $\mathrm{Pb}$ reaction and cleavage that were observed during $\mathrm{Pb}$ detection in the microchannel. Any substrate DNA that is non-specifically bound to the enzyme in the channel would be eluted along with the enzyme and then released in solution. This method will also eliminate challenges involved in surface fluorescence detection such as non-uniformity of signal intensities on the surface, uncertainty in sampling points, and poor reproducibility.

5. Fabrication of the valves and inlets that enable the interface between the microfluidic regime and the outside world. The inlet must be sufficiently engineered to provide onboard filtration of samples.

6. Development of a prototype device that is portable enough so that detection can occur in the field at the source of potential analyte concentrations, such as a river. Our goal is to keep the device small and selfsufficient while maximizing its applications. It remains possible that the use of fiber optics may be required to carry the photons to and from the monitoring device to a master device so that the prototype can be used in remote or difficult-to-reach sites. Finally, the monitoring device must relay the signals to a data logger or controlling unit for data storage, manipulation, and communication. 
7. Address the issue that the chips we manufactured were complex and assembled out of too many materials. For example, electrophoresis in well-defined, fused silica capillaries tends to be highly reproducible. The surface chemistry of silica is well defined and so one expects a well-defined electrophoretic mobility of each analyte if suitable care is taken in terms of electrolyte composition. In our devices, the surface was either PDMS (with a variable charge density) or a hybrid device consisting of layers of PMMA and polycarbonate (the molecular gate) with adhesive in the corners. Thus, the electroosmotic flow and adsorption properties of the analytes were hard to define and also variable. These materials are not optimal for high-quality separations. We determined that the surface properties even change as a function of device age and prior use. This makes defining an optimum set of operating conditions difficult. More effort should go into making devices out of silica or silicon as Shannon describes (2010). While Shannon developed such devices for other applications, the devices should allow reproducible operation. Once the device characteristics are reproducible, then the individual stages can be optimized. This would allow regeneration and multielement operation. 


\section{Literature Cited in Report}

Aggarwal, S. K., M. Kinter, and D.A. Herold. 1994. Determination of lead in urine and whole blood by stable isotope dilution gas chromatography-mass spectrometry. Clinical Chemistry 40 (8): 1494-1502.

Backhouse, C., M. Caamano, F. Oaks, E. Nordman, A. Carrillo, B. J ohnson, and S. Bay. 2000. DNA sequencing in a monolithic microchannel device. Electrophoresis 21: $150-6$.

Basu, S., and M.M. Sharma. 1997. An improved Space-Charge model for flow through charged microporous membranes. J ournal of Membrane Science 124 (1): 77-91.

Breaker, R. R. 1997. DNA enzymes. Nature Biotechnology 15 (5): 427-431. doi: 10.1038/nbt0597-427.

Breaker, R. R., and G.F. J oyce. 1994. A DNA enzyme that cleaves RNA. Chemistry and Biology (4) 1: 223- 9.

Centers for Disease Control (CDC). 1991. Preventing lead poisoning in young children: A statement, [4th revision] ed. U.S. CDC: Atlanta, GA.

Chang, I.-H., J J . Tulock, J . Liu, D.M. Cannon J r., Y. Lu, P.W. Bohn, J .V. Sweedler, and D.M. Cropek. 2005. Miniaturized lead sensor based on lead-specific DNAzyme in a nanocapillary interconnected microfluidic device. Environmental Science and Technology 39: 3756- 61.

Chen, Z., Y. Gao, R. Su, C. Li, and J . Lin. 2006. Fabrication and characterization of poly(methyl methacrylate) microchannels by in situ polymerization with a novel metal template. Electrophoresis 24 (18): 3246- 52.

Christel, L. A., K. Petersen, W. McMillan, and G.T.A. Kovacs. 1998. High aspect ratio silicon microstructures for nucleic acid extraction. In Proceedings of the SolidState Sensor and Actuator Workshop, held J une 8- 11 in Hilton Head, South Carolina, pp. 363- 366.

Cuenoud, B., and J . W. Szostak. 1995. A DNA metalloenzyme with DNA ligase activity. Nature 375(6532):611- 14.

Czarnik, A. W.1995. Desperately seeking sensors. Chemistry and Biology 2(7):423-428.

Flachsbart, B. R., K. Wong, J.M. Iannacone, E.N. Abante, R.L. Vlach, P.A. Rauchfuss, P.W. Bohn, J.V. Sweedler, and M.A. Shannon. 2006. Design and fabrication of a multilayered polymer microfluidic chip with nanofluidic interconnects via adhesive contact printing. Lab on a Chip 6:667- 74.

Folch, A., A. Ayon, O. Hurtado, M.A. Schmidt, and M.J . Toner. 1999. Molding of deep polydimethylsiloxane microstructures for microfluidics and biological applications. J ournal of Biomechanical Engineering 121(1):28- 34. 
Harrison, D. J ., K. Fluri, K. Seiler, Z. Fan, C.S. Effenhauser, and A. Manz. 1993.

Micromachining a miniaturized capillary electrophoresis-based chemical analysis system on a chip. Science 261:895897.

Herne, T. M., and M. J . Tarlov. 1997. Characterization of DNA probes immobilized on gold surfaces. J ournal of the American Chemical Society 119 (38): 8916-8920.

J acobson, Stephen C., Lance B. Koutny, Roland Hergenroeder, Alvin W. Moore, and J.Michael Ramsey. 1994. Analytical Chemistry 66(20):3472- 6.

Jagner, D., L. Renman, Y. Wang. 1994. Determination of lead in microliter amounts of whole blood by stripping potentiometry. Electroanalysis 6(4):285- 291.

J haveri, S.D., R.Kirby, R. Conrad, E.J . Maglott, M. Bowser, R.T. Kennedy, G. Glick, and A.D.J . Ellington. 2000. Designed signaling aptamers that transduce molecular recognition to changes in fluorescence intensity. J ournal of the American Chemical Society 122(11):2469- 2473.

Kemery, P.J ., J .K. Steehler, and P.W. Bohn. 1998. Electric field mediated transport in nanometer diameter channels. Langmuir 14: 2884-9.

Kim, K. J ., and P.V. Stevens. 1997. Hydraulic and surface characteristics of membranes with parallel cylindrical pores. J ournal of Membrane Science 123(2):303- 14. doi:10.1016/ S0376-7388(96)00232-3

Koch, M., C.G.S. Schabmueller, A.G.R. Evans, and A. Brunnschweiler. 1999. Micromachined chemical reaction system. Sensors \& Actuators A 74(1):207- 10.

Koizumi, M., J . N. Q.Kerr, G.A. Soukup, and R.R. Breaker. 1999. Allosteric ribozymes sensitive to the second messengers cAMP and cGMP. Nucleic Acids Symposium Series (26th Symposium on Nucleic Acids Chemistry) 42:275- 276.

Kricka, Larry J . 1998. Revolution on a square centimeter. Nature Biotech. 16:513- 14. doi:10.1038/nbt0698-513.

Kuo, T. C., D.M. Cannon J r., W. Feng, M.A. Shannon, J .V. Sweedler, P.W. Bohn. 2003. Hybrid three-dimensional nanofluidic/microfluidic devices using molecular gates. Sensors and Actuators A: Physical 102(3):223- 33

Li, J ing, and Yi Lu. 2000. A highly sensitive and selective catalytic DNA biosensor for lead ions. J ournal of the American Chemical Society, 122(42):10466- 67. doi: $10.1021 / \mathrm{ja0021316.}$

Li, J., W. Zheng, A. H. Kwon, and Y. Lu. 2000. In vitro selection and characterization of a highly efficient Zn(II)-dependent RNA-cleaving deoxyribozyme. Nucleic Acids Research 28(2):481-8.

Liu, J uewen, Andrea K. Brown, Xiangli Meng, Donald M. Cropek, J onathan D. Istok, David B. Watson, and Yi Lu. 2007. A catalytic beacon sensor for uranium with parts-per-trillion sensitivity and millionfold selectivity. Proceedings of the National Academy of Science 104(7):2056- 2061. doi: 10.1073/pnas.0607875104. 
Manz, A., N. Graber, and H.M. Widmer. 1990. Miniaturized total chemical analysis systems - a novel concept for chemical sensing. Sensors and Actuators B: Chemical 1:244- 8.

Manz, A., D.J . Harrison, D.M.J . Verpoorte, J .C. Fettinger, A. Paulus, H. Ludi, and H.M. Widmer. 1992. Planar chips technology for miniaturization and integration of separation techniques into monitoring systems: Capillary electrophoresis on a chip J ournal of Chromatography B 593(1-2): 253- 8.

McDonald, J . C., D.C. Duffy, D.T. Chio, H. Wu, O.J .A. Schueller, and G.M. Whitesides. 2000. Fabrication of microfluidic systems in poly(dimethylsiloxane) Electrophoresis 21:27- 40 .

Oehme, I., and O.S. Wolfbeis. 1997. Optical sensors for determination of heavy metal ions. Mikrochimica Acta 126(3-4):177-92.

Pan, T., and O.C. Uhlenbeck. 1992. A small metalloribozyme with a two-step mechanism. Nature 358:560-63. doi:10.1038/358560a0.

Parsons, P. J ., and W. Slavin. 1993. A rapid Zeeman graphite furnace atomic absorption spectrometric method for the determination of lead in blood. Spectrochimica Acta Part B 48:925- 39.

Potyrailo, R. A., R.C. Conrad, A.D. Ellington, and G.M. Hieftje. 1998. Adapting selected nucleic acid ligands (aptamers) to biosensors. Analytical Chemistry 70(16):3419-25. doi: 10.1021/ac9802325.

Robertson, M. P., and A.D. Ellington. 1999. In vitro selection of an allosteric ribozyme that transduces analytes to amplicons. Nature Biotechnology 17(1):62-6.

Ruano, J . M., D. Ortega, J .R. Bonar, A.J . McLaughlin, M.G. J ubber, J .M. Cooper, and J .S. Aitchison. 1999. Fabrication of integrated microanalytical chambers and channels for biological assays using flame hydrolysis deposition glass. Microelectric Engineering 46(1):419- 22.

Saksena, S., and A.L. Zydney. 1995. Pore size distribution effects on electrokinetic phenomena in semipermeable membranes. J ournal of Membrane Science 105(3):203- 215.

Santoro, S.W., G.F. J oyce, K. Sakthivel, S. Gramatikova, and C.F. Barbas III. 2000. RNA cleavage by a DNA enzyme with extended chemical functionality. J ournal of the American Chemical Society 122(11):2433-2439.

Shannon, M. 2010. Water desalination: Fresh for less. Nature Nanotechnology 5:24850. doi:10.1038/nnano.2010.71

Steel, A. B., T. M. Herne, M. J .Tarlov. 1998. Electrochemical quantitation of DNA immobilized on gold. Analytical Chemistry 70(22):4670- 7.

Terry, S. C., J .H. J ermann, J . B. Angell. 1979. A gas chromatographic air analyzer fabricated on a silicon wafer. IEEE Trans. Electron Devices 26(12):1880-86. 
Tsien, R. Y. 1993. Fluorescent and photochemical probes of dynamic biochemical signals inside living cells. In ACS Symposium Series 538 (from 204th National Meeting): 130- 146, ed. A.W. Czarnik. Washington, DC: American Chemical Society.

U.S. Environmental Protection Agency (USEPA). 1996. Method 3050B: Acid digestion of sediments, sludges, and soils. Washington, DC: USEPA. Available at: http://www.epa.gov/waste/hazard/testmethods/sw846/pdfs/3050b.pdf (accessed $10 \mathrm{~J}$ une 2010).

Woolley, A. T., and R. A. Mathies. 1994. Ultra-high-speed DNA fragment separations using microfabricated capillary array electrophoresis chips. In Proceedings of the National Academy of Sciences of the United States of America 91:11348- 52. 


\section{Appendix B: List of Publications Produced During the Project}

\section{Peer-reviewed journals}

Chang, I.H., J J . Tulock, J . Liu, W.S. Kim, D.M. Cannon, J r., Y. Lu, P.W. Bohn, J .V. Sweedler, and D.M. Cropek. 2005. Miniaturized lead sensor based on leadspecific DNAzyme in a nanocapillary interconnected microfluidic device. Environmental Science \& Technology 39:3756- 61.

Dalavoy, T.S., D.P. Wernette, M. Gong, J.V. Sweedler, Y. Lu, B.R. Flachsbart, M.A. Shannon, P.W. Bohn, and D.M. Cropek. 2008. Immobilization of DNAzyme catalytic beacons on PMMA for $\mathrm{Pb}^{2+}$ detection. Lab on a Chip 8:786- 93.

Gatimu, E.N., T.L. King, J.V. Sweedler, and P.W. Bohn. 2007. Three-dimensional integrated microfluidic architectures enabled through electrically switchable nanocapillary array membranes. Biomicrofluidics 1(2): 21502. doi:10.1063/1.2732208

Gong, M., B. Y. Kim, B. R. Flachsbart, M. A. Shannon, P. W. Bohn, and J . V. Sweedler. 2008. An on-chip fluorogenic enzyme assay using a multilayer microchip interconnected with nanocapillary array membranes. IEEE Sensors J ournal 8:601- 7.

Gong, M., B. R. Flachsbart, M. A. Shannon, P. W. Bohn, and J. V. Sweedler. 2008. Fluidic communication between multiple vertically segregated microfluidic channels connected by nanocapillary array membranes. Electrophoresis 29:1237- 44 .

Kim, B. Y., C. B. Swearingen, J . A. Ho, E. V. Romanova, P. W. Bohn, and J . V. Sweedler. 2007. Direct immobilization of Fab' in nanocapillaries for manipulating masslimited samples. J ournal of the American Chemical Society 129:7620-6.

Kim, B.Y., J. Yang, M. Gong, B.R. Flachsbart, M.A. Shannon, P. W. Bohn, and J.V. Sweedler. 2009. Multidimensional separation of chiral amino acid mixtures in a multilayered three dimensional hybrid microfluidic/ nanofluidic device. Analytical Chemistry 81:2715- 22.

Piruska, A., S.P. Branagan, D.M. Cropek, J.V. Sweedler, and P.W. Bohn. 2008. Electrokinetically driven fluidic transport in integrated three-dimensional microfluidic devices incorporating gold-coated nanocapillary array membranes. Lab on a Chip 8: 1625- 1631.

Piruska, Aigars, Sean P. Branagan, Alexandra B. Minnis, Zhen Wang, Donald M. Cropek, J onathan V. Sweedler, and Paul W. Bohn. 2010. Electrokinetic control of fluid transport in gold coated nanocapillary array membranes in hybrid nanofluidicmicrofluidic devices. Lab on a Chip 10: 1237- 1244. doi: 10.1039/b924164g.

Prakash, S., A. Piruska, E.N. Gatimu, P. W. Bohn, J.V. Sweedler, and M.A. Shannon. 2008. Nanofluidics: Systems and applications. IEEE Sensors J ournal 8:441- 50. 
Swearingen, C.B., D. P. Wernette, D.M. Cropek, Y. Lu, J.V. Sweedler, and P.W. Bohn. 2005. Immobilization of a catalytic DNA molecular beacon on $\mathrm{Au}$ for $\mathrm{Pb}^{2+}$ detection. Analytical Chemistry 77:442-8.

Wernette, D.P., C.B. Swearingen, D.M. Cropek, Y. Lu, J.V. Sweedler, and P.W. Bohn. 2006. Incorporation of a DNAzyme into Au-coated nanocapillary array membranes with an internal standard for $\mathrm{Pb}(\mathrm{II})$ sensing. The Analyst 131:41- 7.

\section{Technical reports}

Cropek, Donald M., Tulika S. Dalavoy, Paul W. Bohn, Yi Lu, J onathan Sweedler, and Mark Shannon. 2011. Metal ion sensor with catalytic DNA in a nanofluidic intelligent processor. ERDC/ CERL TR-11-47. Champaign, IL: U.S. Army Engineer Research and Development Center- Construction Engineering Research Laboratory.

\section{Book chapters}

Iannacone, J. M., B. Y. Kim, T. L. King, J. V. Sweedler, and P. W. Bohn. 2008. Manipulating mass limited samples using hybrid microfluidic/ nanofluidic networks. In Biological Applications of Microfluidics, 1st Ed. ed. F.A. Gomez, 45170. Maiden, MA: Wiley Interscience.

\section{Conference proceedings}

Chang, In-Hyoung, J oseph Tulock, Donald M. Cannon, Carla Swearingen, J uewen Liu, Yi Lu, Donald M. Cropek, Paul W. Bohn, and J onathan V. Sweedler. 2004. Development of miniaturized lead sensors using catalytic DNA in an on-chip capillary electrophoresis format. Presented at Pittcon 2004, held March 2004 in Chicago, IL.

Cropek, D.M. 2005. Advanced catalytic DNA biosensors. Richmond, VA: Virginia Commonwealth University, August 3, 2005 lecture.

Cropek, D.M. 2005. Immobilization of lead-specific DNAzyme on a microfluidic device for $\mathrm{Pb}$ (II) detection. Invited lecture, Fall meeting of Materials Research Society held Nov 28, 2005, in Boston, MA.

. 2005. Microfluidic/ nanofluidic sensors incorporating catalytic DNA for detection of heavy metal analytes. Invited lecture at Materials Research Society MURI Workshop, held Oct 5, 2005, at the Beckman Institute, University of Illinois, Champaign, IL.

.2005. Microfluidic lead sensor incorporating catalytic DNA. Invited lecture, Federal Remediation Technologies Roundtable, held Dec 7, 2005, in Arlington, VA.

. 2007. Microfluidic/nanofluidic sensors using catalytic DNA for heavy metal detection. Presented at 233rd ACS Meeting, held 27 March 2007 in Chicago, IL.

. 2007. Microfluidic/ nanofluidic sensors using catalytic DNA for heavy metal detection. Presented at AIChE Annual Conference, held 6 November 2007 in Salt Lake City, UT. 
Cropek, Donald M., Kris Wang, Yi Lu, Bruce Flachsbart, Mark A. Shannon, J onathan V. Sweedler, and Paul W. Bohn. 2006. Next generation sensors for contaminants in water: Catalytic DNA as a molecular beacon. Poster and manuscript presented at 25th Army Science Conference, Dec 2006, Orlando, FL.

Cropek, Donald M., Yi Lu, Mark A. Shannon, J onathan V. Sweedler, Paul W. Bohn. 2008. Microfluidic/ nanofluidic sensors using catalytic DNA for heavy metal detection. Presented at Rocky Mountain Analytical Conference, held J uly 28, 2008 in Breckinridge, CO.

Dalavoy, Tulika, Yi Lu, Mark A. Shannon, J onathan V. Sweedler, Paul W. Bohn, and Donald M. Cropek. 2008. Microfluidic/nanofluidic sensors using catalytic DNA for heavy metal detection. Pittcon 2008, held March 2008 in New Orleans, LA.

Wang, Xiaofeng, Paul W. Bohn, Charles S. Henry, Yi Lu, J onathan V. Sweedler, and Donald M. Cropek. 2006. Microfluidic chip for rapid analysis of heavy metals. Bioanalytical Sensors at Gordon Conference, held Feb 25, 2006 in Ventura, CA.

Wernette, Daryl, Carla Swearingen, Yi Lu, J onathan V. Sweedler, Paul W. Bohn and Donald M. Cropek. 2007. Incorporation of a DNAzyme into Au-coated nanocapillary array membranes with an internal standard for $\mathrm{Pb}^{2+}$ sensing. ACS WaterCAMPWS meeting, held March 25- 29, 2007, in Chicago, IL. 


\section{Appendix C: Other Supporting Material}

2005 Army Research \& Development Achievement Award: "Basic and Applied Research in Microfluidic Heavy Metal Ion Sensors to Support Environmental Long Term Monitoring."

This research team represents a model effort to combine ERDC researchers with the best academic scientists to perform recognized basic and applied research. The environmental sensors developed here demonstrate the high sensitivity and selectivity required for long term monitoring devices. The wide breadth of scientific disciplines included on this team emphasizes the intellectual diversity needed for successful research efforts today. 


\section{Acronyms and Abbreviations}

\begin{tabular}{|c|c|}
\hline Term & Spellout \\
\hline AC & alternating current \\
\hline ASJ & atom-scale junctions \\
\hline Am & amino \\
\hline $\mathrm{Au}$ & gold \\
\hline BGE & background electrolyte \\
\hline CDC & Centers for Disease Control \\
\hline Co (II) & cobalt \\
\hline $\mathrm{COOH}$ & carboxylic acid \\
\hline CTE & coefficient of thermal expansion \\
\hline CTAB & cetyl trimethylammonium bromide \\
\hline $\mathrm{Cu}$ & copper \\
\hline Су3, Су5 & reactive water-soluble fluorescent dyes of the cyanine dye family \\
\hline dd & deionized and distilled (water) \\
\hline DI & deionized water \\
\hline DoD & Department of Defense \\
\hline DNA & deoxyribonucleic acid \\
\hline DNAzyme & molecules of DNA that possess enzymatic activity \\
\hline ECD & electrodeposition \\
\hline ECMI & Environmental Characterization and Monitoring Initiative \\
\hline EDC & 1-ethyl-3-(3-dimethylaminopropyl)-carbodiimide \\
\hline EOF & electroosmotic flow \\
\hline ERDC-CERL & $\begin{array}{l}\text { Engineer Research and Development Center-Construction Engineering Research } \\
\text { Laboratory }\end{array}$ \\
\hline ESI & electrospray ionization \\
\hline Fab & fragment of antibody \\
\hline $\mathrm{FI}$ & fluorescein \\
\hline FITC & fluorescein isothiocyanate \\
\hline GFP & green fluorescent protein \\
\hline HEPES & $\mathrm{N}$-[2-hydroxyethyl]piperazine-N'-[2-ethanesulfonic acid] \\
\hline HPLC & high-performance liquid chromatography \\
\hline$\kappa$ & inverse Debye length \\
\hline LIF & laser-induced fluorescence \\
\hline $\mathrm{mM}$ & millimolar \\
\hline $\mathrm{MCH}$ & mercaptohexanol \\
\hline MEMS & microelectro-mechanical systems \\
\hline MS & mass spectrometry \\
\hline $\mathrm{NaBH} 4$ & sodium borohydride \\
\hline
\end{tabular}




$\begin{array}{ll}\text { Term } & \text { Spellout } \\ \text { NaCl } & \text { sodium chloride } \\ \text { NAI } & \text { nanocapillary array interconnect } \\ \text { NCA } & \text { nanocapillary array } \\ \text { NCAM } & \text { nanocapillary array membranes } \\ \text { NIP } & \text { nanofluidic intelligent processor } \\ \text { PB } & \text { phosphate buffer } \\ \text { Pb } & \text { lead } \\ \text { Pb2+ } & \text { cationic lead } \\ \text { PBS } & \text { phosphate-buffered saline } \\ \text { PCR } & \text { polymerase chain reaction } \\ \text { PCTE } & \text { polycarbonate nuclear track-etched membrane } \\ \text { PDMS } & \text { polydimethylsiloxane, part of a group of polymeric organosilicon compounds } \\ & \text { that are commonly referred to as silicones } \\ \text { PMMA } & \text { poly(methylmethacrylate) } \\ \text { PMT } & \text { photomultiplier tube } \\ \text { Pt } & \text { platinum } \\ \text { RCRA } & \text { Resource Conservation and Recovery Act } \\ \text { RIE } & \text { reactive ion etching } \\ \text { SDS } & \text { sodium dodecyl sulfate } \\ \text { SEED } & \text { SERDP Exploratory Development } \\ \text { SEMP } & \text { Strategic Environmental Management Program } \\ \text { SERDP } & \text { Strategic Environmental Research \& Development Program } \\ \text { SiO } 2 & \text { silicon dioxide } \\ \text { SON } & \text { Statement of Need } \\ \text { SSG } & \text { superstreptavidin glass } \\ \text { SU8 } & \text { epoxy-based negative photoresist used mainly in microfluidic fabrications } \\ \text { TAMRA } & \text { carboxytetramethylrhodamine } \\ \text { TCEP } & \text { tris (2-carboxyethyl) phosphine } \\ \text { TSCA } & \text { Toxic Substances Control Act } \\ \text { UO }{ }_{2}^{2+} & \text { uranyl ion; stable form in groundwater } \\ \text { USEPA } & \text { United States Environmental Protection Agency } \\ \text { UV } & \text { Zn }{ }^{2+}\end{array}$




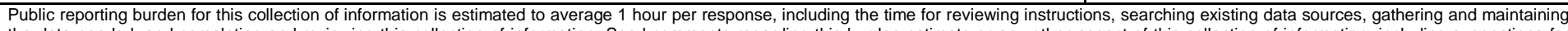

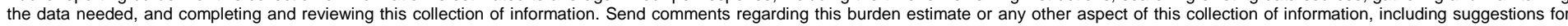

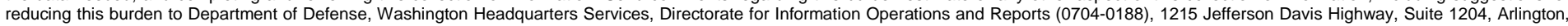

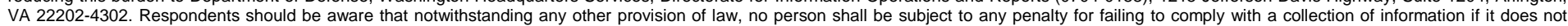
display a currently valid OMB control number. PLEASE DO NOT RETURN YOUR FORM TO THE ABOVE ADDRESS.
1. REPORT DATE (DD-MM-YYYY)
2. REPORT TYPE
3. DATES COVERED (From - To)

December 2011

4. TITLE AND SUBTITLE

Final Technical Report

Metal Ion Sensor with Catalytic DNA in a Nanofluidic Intelligent Processor

5a. CONTRACT NUMBER

5b. GRANT NUMBER

5c. PROGRAM ELEMENT NUMBER

\section{AUTHOR(S)}

Donald M. Cropek, Tulika S. Dalavoy, Paul W. Bohn, Yi Lu, Jonathan V. Sweedler, and Mark A. Shannon

5d. PROJECT NUMBER

ER-1459

5e. TASK NUMBER

5f. WORK UNIT NUMBER

8. PERFORMING ORGANIZATION REPORT NUMBER

ERDC/CERL TR-11-47

U.S. Army Engineer Research and Development Center

Construction Engineering Research Laboratory

P.O. Box 9005

Champaign, IL 61826-9005

9. SPONSORING I MONITORING AGENCY NAME(S) AND ADDRESS(ES)

Strategic Environmental Research and Development Program (SERDP)

901 North Stuart Street, Suite 303

Arlington, VA 22203

\section{DISTRIBUTION / AVAILABILITY STATEMENT}

Approved for public release; distribution is unlimited.

\section{SUPPLEMENTARY NOTES}

\section{ABSTRACT}

Due to the numerous toxicological effects of lead, its presence in the environment needs to be effectively monitored. Incorporating a biosensing element within a microfluidic platform enables rapid and reliable determinations of lead at trace levels. This work produced a microchip-based lead sensor that employs a lead-specific DNAzyme (also called catalytic DNA or deoxyribozyme) as a recognition element that cleaves its complementary substrate DNA strand only in the presence of cationic lead (Pb2 ${ }^{+}$). Fluorescent tags on the DNAzyme translate the cleavage events to measurable, optical signals proportional to Pb2 ${ }^{+}$concentration. The DNAzyme responds sensitively and selectively to $\mathrm{Pb2}^{+}$, and immobilizing the DNAzyme in the sensor permits both sensor regeneration and localization of the detection zone. The immobilized DNAzyme retains its $\mathrm{Pb}^{+}$detection activity in the microfluidic device and can be regenerated and reused. The particular DNAzyme shows no response to other common metal cations and the presence of these contaminants does not interfere with the lead-induced fluorescence signal. Attempts were made to incorporate a second DNAzyme with selectivity for uranium in the same microfluidic chip sensor, to demonstrate multiplexing capabilities for multiple metal analytes in a single injection. Crosstalk between the lead DNAzyme and the uranium DNAzyme severely limited the utility of this multi-analytic chip.

\section{SUBJECT TERMS}

Catalytic DNA, DNAzyme, heavy metals, lead, sensors

\begin{tabular}{|c|c|c|c|c|c|}
\hline \multicolumn{3}{|c|}{ 16. SECURITY CLASSIFICATION OF: } & \multirow{2}{*}{$\begin{array}{c}\text { 17. LIMITATION } \\
\text { OF ABSTRACT } \\
\text { UU }\end{array}$} & \multirow{2}{*}{$\begin{array}{l}\text { 18. NUMBER } \\
\text { OF PAGES } \\
127\end{array}$} & \multirow{2}{*}{$\begin{array}{l}\text { 19a. NAME OF RESPONSIBLE } \\
\text { PERSON } \\
\begin{array}{l}\text { 19b. TELEPHONE NUMBER (include } \\
\text { area code) }\end{array}\end{array}$} \\
\hline $\begin{array}{l}\text { a. REPORT } \\
\text { Unclassified }\end{array}$ & $\begin{array}{l}\text { b. ABSTRACT } \\
\text { Unclassified }\end{array}$ & $\begin{array}{l}\text { c. THIS PAGE } \\
\text { Unclassified }\end{array}$ & & & \\
\hline
\end{tabular}

